The Astrophysical Journal Supplement Series, 173:315-341, 2007 December

(C) 2007. The American Astronomical Society. All rights reserved. Printed in U.S.A.

\title{
THE UV-OPTICAL COLOR MAGNITUDE DIAGRAM. II. PHYSICAL PROPERTIES AND MORPHOLOGICAL EVOLUTION ON AND OFF OF A STAR-FORMING SEQUENCE
}

\author{
David Schiminovich, ${ }^{1}$ Ted K. Wyder, ${ }^{2}$ D. Christopher Martin, ${ }^{2}$ Benjamin. D. Johnson, ${ }^{1}$ Samir Salim, ${ }^{3,4}$ Mark Seibert, ${ }^{5}$ \\ Marie A. Treyer, ${ }^{2,6}$ Tamás Budavári, ${ }^{7}$ Charles Hoopes, ${ }^{7}$ Michel Zamojski, ${ }^{1}$ Tom A. Barlow, ${ }^{2}$ Karl G. Forster, ${ }^{2}$ \\ Peter G. Friedman, ${ }^{2}$ Patrick Morrissey, ${ }^{2}$ Susan G. Neff, ${ }^{8}$ Todd A. Small, ${ }^{2}$ Luciana Bianchi, ${ }^{9} J_{\text {José Donas, }}{ }^{6}$ \\ Timothy M. Heckman, ${ }^{7}$ Young-Wook Lee, ${ }^{10}$ Barry F. Madore, ${ }^{5}$ Bruno Milliard, ${ }^{6}$ R. Michael Rich, ${ }^{4}$ \\ Alex. S. Szalay, ${ }^{7}$ Barry Y. Welsh, ${ }^{11}$ and Sukyoung Y Y ${ }^{10}$ \\ Received 2007 August 2; accepted 2007 October 16
}

\begin{abstract}
We use the UV-optical color magnitude diagram in combination with spectroscopic and photometric measurements derived from the SDSS spectroscopic sample to measure the distribution of galaxies in the local universe $(z<0.25)$ and their physical properties as a function of specific star formation rate $\left(\mathrm{SFR} / M_{\star}\right)$ and stellar mass $\left(M_{\star}\right)$. Throughout this study our emphasis is on the properties of galaxies on and off of a local "star-forming sequence." We discuss how the physical characteristics of galaxies along this sequence are related to scaling relations typically derived for galaxies of different morphological types. We find, among other trends, that our measure of the star formation rate surface density, $\Sigma_{\text {SFR }}$, is nearly constant along this sequence. We discuss this result and implications for galaxies at higher redshift. For the first time, we report on measurements of the local UV luminosity function versus galaxy structural parameters, as well as inclination. We also split our sample into disk-dominated and bulge-dominated subsamples using the $i$-band Sersic index and find that disk-dominated galaxies occupy a very tight locus in SFR $/ M_{\star}$ vs. $M_{\star}$ space, while bulge-dominated galaxies display a much larger spread of SFR $/ M_{\star}$ at fixed stellar mass. A significant fraction of galaxies with SFR $/ M_{\star}$ and $\Sigma_{\text {SFR }}$ above those on the "star-forming sequence" are bulge-dominated. We can use our derived distribution functions to ask whether a significant fraction of these galaxies may be experiencing a final episode of star formation ( possibly induced by a merger or other burst), soon to be quenched, by determining whether this population can explain the growth rate of the non-star-forming galaxies on the "red sequence." We find that this is a plausible scenario for bulge-dominated galaxies near the characteristic transition mass under reasonable assumptions regarding quenching timescales. Similarly, we use this technique to estimate the rate of mergers/starbursts that take galaxies off of the star-forming sequence and show that the implied merger rates are consistent with local measurements.
\end{abstract}

Subject headings: galaxies: evolution — galaxies: formation — surveys — ultraviolet: galaxies

\section{INTRODUCTION}

What determines the star formation rate (SFR) of a galaxy? Ample evidence suggests that it is the quantity and distribution of cold gas (Schmidt 1959; Kennicutt 1998a) and the gas-dynamical processes responsible for triggering, regulating, or quenching new star formation. In the context of a hierarchical clustering scenario for galaxy formation (e.g., Kauffmann et al. 1993), these mech-

\footnotetext{
1 Department of Astronomy, Columbia University, 550 West 120th Street, New York, NY 10027; ds@astro.columbia.edu.

2 California Institute of Technology, MC 405-47, 1200 East California Boulevard, Pasadena, CA 91125.

3 NOAO, 950 North Cherry Avenue, Tucson, AZ 85719.

4 Department of Physics and Astronomy, University of California, Los Angeles, CA 90095

5 Observatories of the Carnegie Institution of Washington, 813 Santa Barbara Street, Pasadena, CA 91101.

${ }^{6}$ Laboratoire d'Astrophysique de Marseille, BP8, Traverse du Siphon, F-13376 Marseille, France.

7 Department of Physics and Astronomy, The Johns Hopkins University, Homewood Campus, Baltimore, MD 21218.

${ }^{8}$ Laboratory for Astronomy and Solar Physics, NASA Goddard Space Flight Center, Greenbelt, MD 20771.

9 Center for Astrophysical Sciences, The Johns Hopkins University, 3400 North Charles Street, Baltimore, MD 21218.

${ }_{10}$ Center for Space Astrophysics, Yonsei University, Seoul 120-749, Korea.

11 Space Sciences Laboratory, University of California at Berkeley, 601 Campbell Hall, Berkeley CA 94720.
}

anisms are necessarily linked to the flow of dark and baryonic matter over a wide range of scales, densities, and temperatures (Kereš et al. 2005). Given this complexity, it is intriguing that the integrated light from many galaxies can be explained using simple star formation histories (SFHs) (Tinsley 1968; Searle et al. 1973; Bruzual \& Charlot 2003; although see also Kauffmann et al. 2007). Such work has led to an apparent understanding of star formation on cosmological scales (Hopkins \& Beacom 2006), although accurate physical models embedded within a realistic framework for galaxy assembly (e.g., Stringer \& Benson 2007) are required to understand star formation in individual galaxies.

Measurements of the colors and structure of a galaxy should guide these models by providing insight into the connection between star formation and assembly. The fact that galaxies in the local universe appear to show a remarkable correlation between their star formation history and their structure-disk-dominated galaxies show higher present to past-averaged star formation rates than bulge-dominated galaxies (Kennicutt 1998b) — would appear to suggest a straightforward link, but we now know that the explanation must be nontrivial (De Lucia et al. 2006). A crucial component of these analyses is a quantitative and representative description of the galaxy population. In this regard, colormagnitude distributions and their derivatives have emerged as useful tools because they can be easily interpreted in terms of the star formation history and the stellar mass content and therefore are easily connected to models of galaxy assembly and the 
buildup of massive galaxies along the "red sequence" (Faber et al. 2007).

In this paper, the second in a series, we explore how the UVoptical properties of a large sample of galaxies in the local universe can be used to understand the distribution of SFR and the connection with assembly history across the population. We accomplish this by expanding on the analysis of the UV-color magnitude diagram ( Wyder et al. 2007, hereafter Paper I) using observations of 26241 galaxies from the Galaxy Evolution Explorer (GALEX) Medium Imaging Survey (MIS), combined with the Sloan Digital Sky Survey (SDSS) primary spectroscopic survey and incorporating additional "value-added" data related to the morphology/structure, star formation history, and dust attenuation in each galaxy. Our analysis has many similarities to recent studies conducted using SDSS (Blanton et al. 2003c; Kauffmann et al. 2003a, 2003b; Shen et al. 2003), local surveys (Driver et al. 2006; Jansen \& Kannappan 2001), and high-z investigations (Bell et al. 2005; Noeske et al. 2007a, 2007b), although it extends those studies in several ways, described below.

As discussed in Paper I, a notable feature of the UV-optical color diagram is the very wide separation between the peaks of the blue and the red galaxy populations. Of central importance is the strongly peaked locus of star-forming blue galaxies that has been variously called the "blue sequence" Blanton (2006) and the "main sequence" Noeske et al. (2007a). Paper I, Noeske et al. (2007a), and Salim et al. (2007, hereafter S07) show that the majority of star-forming galaxies of a given stellar mass possess a narrow range of SFR, a result already noted by Brinchmann et al. (2004), Feulner et al. (2006), and Cattaneo et al. (2007) . This stands in marked contrast to the optical color-based view, which emphasizes a tight "red sequence" and a scattered "blue cloud" (Bell et al. 2005). It suggests strong similarities among starforming galaxies and a greater diversity of SFR for those galaxies that optically appear "red-and-dead." In many respects, this alternate view is reminiscent of the progression from the Hubble classification scheme, with a rich description of spirals and only a few elliptical classes, toward work in recent years that revealed that ellipticals possess a greater range of structure (at low and high spatial frequency) than originally thought (de Zeeuw \& Franx 1991).

Here we quantify the structural and physical properties of a local "star-forming sequence" (SF sequence), defined by a relationship between stellar mass and star formation rate, and use it to understand the characteristics of the dominant galaxy population, including the slope of the sequence itself. Some of this analysis is quite complementary to the work of S07. We also focus on the distribution disk-dominated and bulge-dominated galaxies across the full range of SFR $/ M_{\star}$ and $M_{\star}$, and investigate the structure of outliers to the SF sequence. While in Martin et al. (2007, hereafter Paper III), specific attention has been given to galaxies in the intermediate region between the blue and red galaxy population (the "green valley"), our ultimate focus will be on galaxies with specific star formation rates higher than those on the SF sequence. This is the population in the SFR $/ M_{\star}$ vs. $M_{\star}$ diagram that has evolved most dramatically since $z \sim 1$.

We briefly describe the outline of this paper. After presenting the data in $\S 2$, we investigate in $\S 3$ the physical properties of galaxies on and off of the SF sequence. In $\S 4$ we investigate the relationship between star formation history and structure and its connection with the evolution of galaxies on and off of the SF sequence. Throughout this paper, we make use of the flat $\Lambda \mathrm{CDM}$ cosmology with $H_{0}=70 \mathrm{~km} \mathrm{~s}^{-1} \mathrm{Mpc}^{-1}$ and $\Omega_{\Lambda}=0.7$.

\section{DATA}

\subsection{GALEX DR4-MIS Cross-Match}

$G A L E X$ data were obtained as part of the GALEX Medium Imaging Survey (MIS; Martin et al. 2005), cataloged as part of an internal data release 1.1 (IR1.1) and processed using standard GALEX pipeline processing (Morrissey et al. 2005, 2007). The MIS reaches a limiting UV magnitude of $\sim 23$ through single or multiple eclipse exposures that are typically $1 \mathrm{ks}$ or greater in duration. MIS targets were initially selected to overlap the SDSS Data Release 2 footprint, although some additional overlap with SDSS Data Release 4 (DR4; Adelman-McCarthy et al. 2006) made it advantageous to use the latter release for the cross-match.

A total of 67,883 SDSS DR4 spectroscopic objects were within $0.6^{\circ}$ of the field centers of GALEX MIS observations. For each of these objects we searched for the closest GALEX detection within a $4^{\prime \prime}$ radius. Objects with no match were considered GALEX nondetections. To produce a complete statistical sample, further cuts were applied. SDSS objects were selected from the main galaxy sample with $r$-band magnitudes $14.5<r<17.6$, magnitude error $\sigma_{r}<0.2 \mathrm{mag}$, redshift in the range $0.01<z<0.25$, and redshift confidence $z_{\text {conf }}>0.67$. The sample was limited to regions of sky with UV exposure times greater than $800 \mathrm{~s}$, location on-detector within $0.55^{\circ}$ offield center, an NUV magnitude cut $(16<$ NUV $<23)$ and non-artifacts using nuv_artifact $\leq 1$. These cuts, source matching, and completeness are all described in more detail in Paper I and in Bianchi et al. (2007). The main sample used in this paper contains a total of 26,241/18,091 galaxies detected with NUV/FUV $<23$ over an area of 485.321 / $411.266 \mathrm{deg}^{2}$. For most of our analysis we use the NUVdetected sample ("main galaxy sample"), noting exceptions where appropriate.

We combined our GALEX/SDSS matched photometric catalog with extra derived parameters obtained from the MPA/ JHU (Kauffmann et al. 2003a, 2003b; Brinchmann et al. 2004; Tremonti et al. 2004) and NYU (Blanton et al. 2005b) valueadded catalogs. We briefly discuss the parameters we have used below.

Photometry, redshifts.-For GALEX FUV and NUV photometry we used Kron magnitudes and errors ${ }^{12}$ generated by the IR11 pipeline. These magnitudes were dereddened for Galactic extinction as described in Paper I. We used ugriz Petrosian magnitudes and dereddening values obtained from the SDSS DR4 pipeline. Redshifts and redshift errors were obtained from the Princeton reductions, ${ }^{13}$ which have been subsequently incorporated into the MPA/JHU files.

Galaxy size, light profile shape and model fit.-We used the DR4 pipeline Petrosian 50\% and 90\% radii. Redshifts and assumed cosmology were used to convert these to physical sizes in $\mathrm{kpc}$. These values were used to derive secondary quantities, such as surface densities and concentration. The SDSS pipeline also performs model fits to the galaxy light profile using an exponential model commonly used for fitting spiral disks and a de Vaucouleurs model used for fitting bulges and ellipticals. The pipeline quantity FracDeV (erroneously labeled FracPSF in SDSS output catalogs) provides an estimate for how much light from the galaxy is coming from the bulgelike component. For the bulk of our analysis we made use of the improved seeing-deconvolved, axisymmetric Sersic profile fits from the NYU VAGC (described

\footnotetext{
12 Early versions of the GALEX pipeline occasionally underestimated the true error; corrected in this and later releases.

13 See http://spectro.princeton.edu.
} 

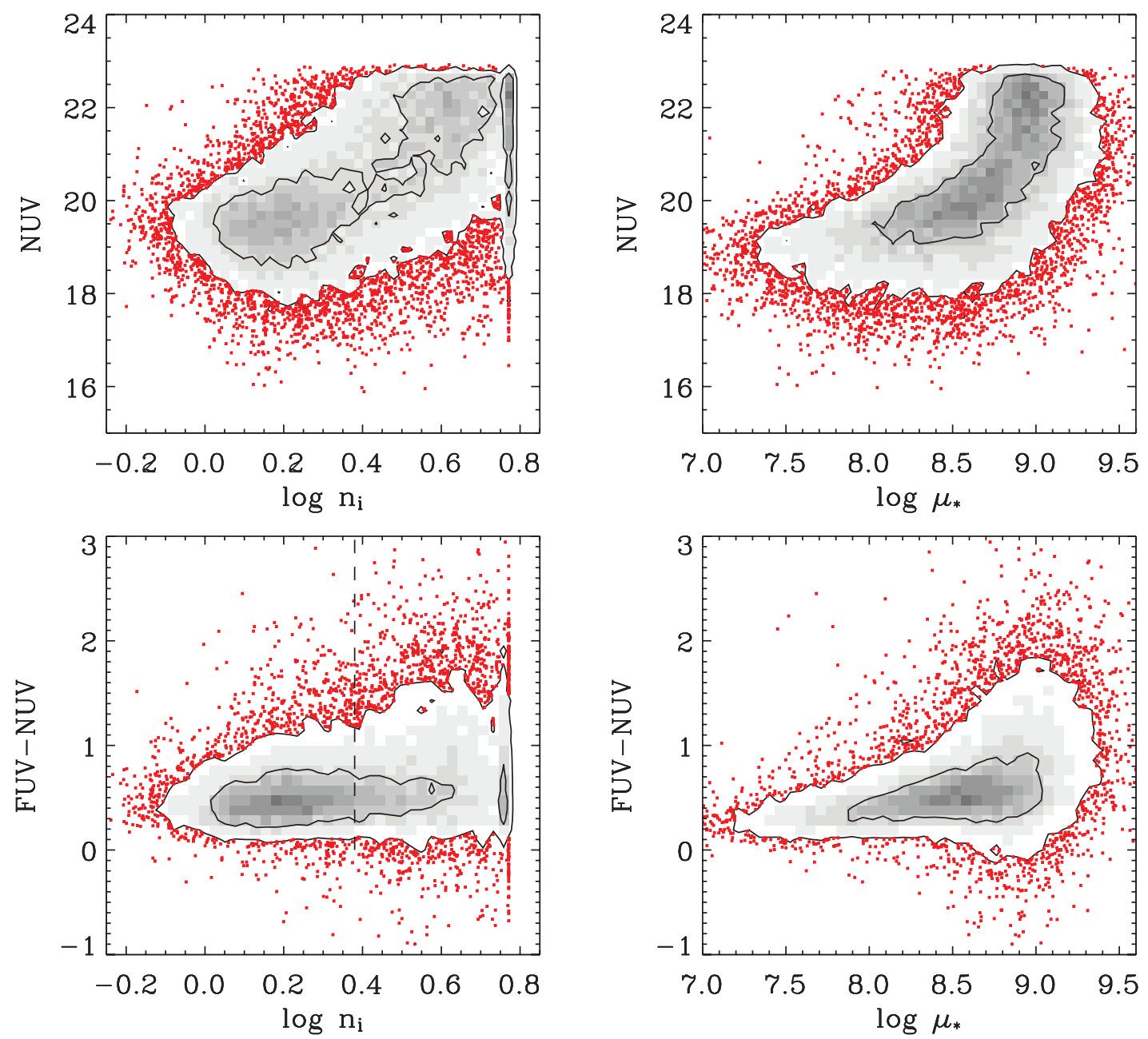

FIg. 1.-Main galaxy sample bivariate number density distribution. Top: NUV magnitude and FUV -NUV color vs. $\log n_{i}$. Bottom: NUV and FUV-NUV vs. $\log \mu_{\star}$. Contours enclose $50 \%$ and $90 \%$ of the distribution, with outliers plotted individually.

in the appendix of Blanton et al. 2005b). The Sersic profile has the form

$$
I(r)=A \exp \left[-\left(r / r_{0}\right)^{1 / n}\right]
$$

where the Sersic index $n$ is 1 for an exponential light profile and 4 for a de Vaucouleurs profile. As reported in Blanton et al. (2005b), these fits slightly underestimate high- $n$ galaxies (measuring 3.5 for galaxies with $n=4$ ), but are sufficient for our purposes. We refer the reader to Blanton et al. (2003c) and Blanton et al. (2005b) for further discussion regarding the use of the Sersic index over a similar redshift range. We use only the $i$-band fit, using a longer wavelength band that will be less sensitive to recent star formation, and typically express the Sersic index in logarithmic form $\left(\log n_{i}\right)$. In later sections we also divide our sample into diskdominated and bulge-dominated galaxies at $n_{i}=2.4\left(\log n_{i}=\right.$ 0.38 ). Note that this dividing line is similar to or slightly higher than that used in other analyses. Vincent \& Ryden (2005) separate disk and bulge-dominated galaxies using $n=2.0$, which yields a cut very close to FracDeV $=0.5$. We chose our limit to conservatively restrict the number of disk-dominated galaxies identified as bulge-dominated. We obtain 12,835 disk-dominated and 13,406 bulge-dominated galaxies in our main galaxy sample using this coarse classification.
Stellar mass and stellar mass surface density.-Stellar masses were obtained from the MPA/JHU catalogs using the values discussed in Kauffmann et al. (2003a). Stellar masses and the $z$-band half-light radius were used to derive stellar mass surface densities:

$$
\mu_{\star}=\frac{0.5 M_{\star}}{\pi R_{50, z}^{2}}
$$

in $M_{\odot} \mathrm{kpc}^{-1}$. In Figure 1 we plot the distribution of our sample as a function of measured magnitude, UV color (FUV - NUV), Sersic index, and $\log \mu_{\star}$. Most disk and low stellar mass surface density galaxies $\left(\log \mu_{\star}<8.5\right)$ in our sample are brighter than our magnitude limit, while some bulge-dominated and $\log \mu_{\star}>8.5$ galaxies are fainter than our limit. In the next section we make use of the $\left(1 / V_{\max }\right)$ method in order to correct for this incompleteness.

\subsection{K-Corrections and $V_{\max }$ Calculation}

Our sample has a median redshift of $0.086(0.078 / 0.10$ for disk-dominated/bulge-dominated). Using the kcorrect code (v4.1.4; Blanton \& Roweis 2007), we derived rest-frame absolute magnitudes in the bands ${ }^{0.1} \mathrm{FUV},{ }^{0.1} \mathrm{NUV},{ }^{0.1} u,{ }^{0.1} \mathrm{~g},{ }^{0.1} r,{ }^{0.1} i$, and ${ }^{0.1} z$, generated by shifting the observed bandpasses blueward by a factor in wavelength of $1 /(1+z)(=1 / 1.1$ for $z=0.1)$. This approach, described in Blanton et al. (2003a), minimizes the amplitude of the $K$ correction [beyond a trivial constant 

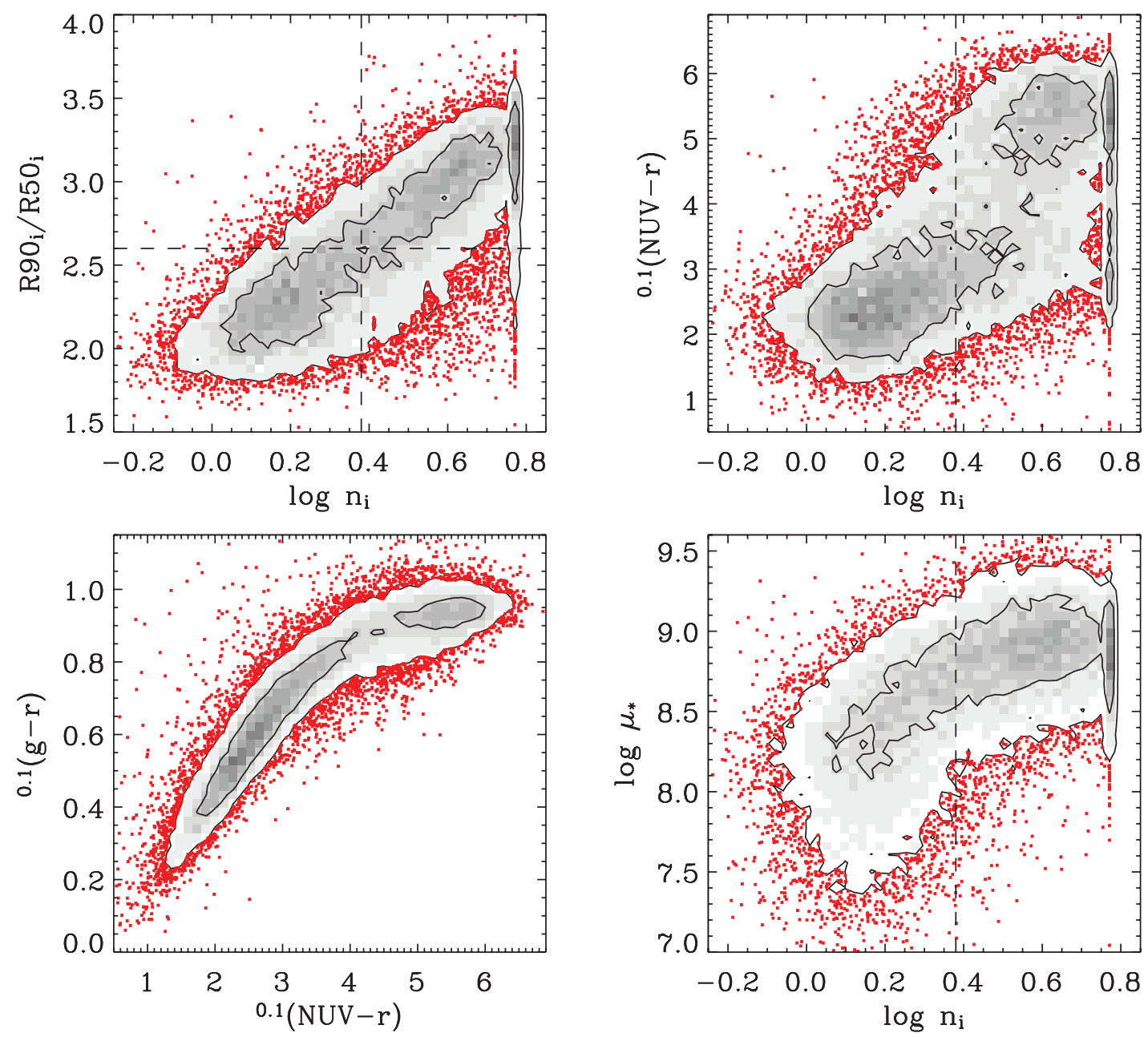

FIG. 2.-Main galaxy sample bivariate number density distribution. Top: $i$-band concentration and NUV $-r$ color vs. $\log n_{i}$. Bottom left: $g-r$ color vs. NUV $-r$. Bottom right: $\log \mu_{\star}$ vs. $\log n_{i}$. Contours enclose $50 \%$ and $90 \%$ of the distribution, with outliers plotted individually.

$\left.-2.5 \log _{10}(1.1)\right]$ determined for the typical galaxy in our sample. We applied these for a given band $b$, using the equation

$$
M_{b, 0.1}=m_{b}-\mathrm{DM}-K_{b, 0.1}(z)+(z-0.1) Q,
$$

where DM is the distance modulus, and $Q=1.6$ (see Paper I for description) is added to account for luminosity evolution over the redshift range being considered (Blanton et al. 2003b).

Figure 2 shows the distribution of galaxies in our sample as a function of rest-frame color and structural parameters. The ${ }^{0.1}(g-r)$ vs. ${ }^{0.1}(\mathrm{NUV}-r)$ color-color diagram demonstrates quite clearly how the NUV $-r$ color covers a much wider magnitude range than $g-r$. In addition, the $g-r$ color starts to saturate for red galaxies, while NUV $-r$ varies by more than 2 magnitudes. We see indications from these plots that rest-frame UV-optical colors correlate with Sersic index (and concentration), although as with the color-color plot, $\log \mu_{\star}$ is nearly constant for bulge-dominated galaxies.

Using our $K$-corrected magnitudes we determined $V_{\max }$, the maximum volume over which the galaxy would have been included in our sample. This was calculated using our adopted cosmology for three bands individually (FUV, NUV, $r$ ), as well as the combination of any two (or all three). For most of our analysis below we use $V_{\max , \mathrm{NUV}}, r$ which is the intersection of the detection volume in each individual band. For any analysis using FUV data, we use all three bands to determine the appropriate $V_{\max }$. Again, the reader is referred to Paper I for a more detailed discussion.

\section{PHYSICAL PROPERTIES ACROSS THE GALAXY POPULATION}

\subsection{A Starting Point: UV Luminosity Function vs. Structural Parameters}

We begin with an example that highlights the issues we will be considering in this paper. In Figure 3 we plot the UV luminosity function calculated from our sample using the $1 / V_{\max }$ method. We show the FUV and NUV luminosity functions split by Sersic index $n_{i}$, where we use the separation described above to define disk-dominated and bulge-dominated samples. We also fit Schechter parameters, which we include in Table 1. The total luminosity function is consistent with the one determined by Wyder et al. (2005) and Treyer et al. (2005), although the Schechter fit does undershoot the most luminous points, some of which might be active galactic nuclei (AGNs).

In Figure 4 we plot the luminosity function split by stellar mass surface density, $\log \mu_{\star}$. Below each plot we show the fractional abundance of bulge-dominated (or high $\log \mu_{\star}$ ) galaxies vs. UV magnitude. We find in both sets of plots (and for both FUV and NUV) that the fraction of bulge-dominated (or high $\log \mu_{\star}$ ) galaxies increases with increasing UV luminosity. These observations 

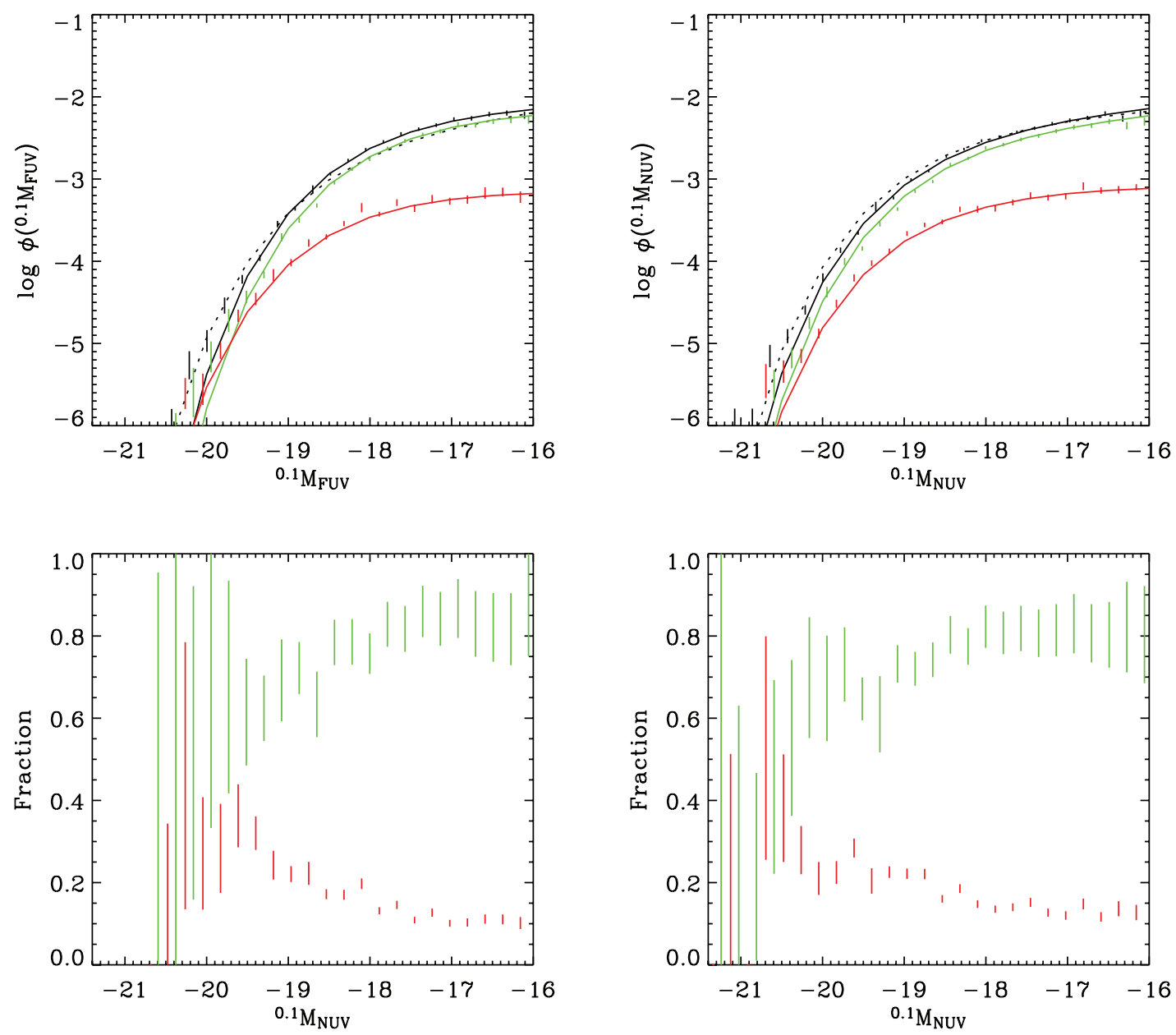

FIG. 3.-Top: FUV and NUV luminosity function for complete sample (black) and disk and bulge-dominated subsamples split by $i$-band Sersic index $n: n_{i}<2.4$ ( $\log n_{i}<$ 0.38) (green) and $n_{i}>2.4\left(\log n_{i}>0.38\right)($ red $)$. Units of $\phi$ are in $\mathrm{Mpc}^{-3} \mathrm{mag}^{-1}$. The dotted curve is from the Wyder et al. (2005) and Treyer et al. (2005) LF. Bottom: Relative fraction (1/Vmax-weighted) of disk vs. total and bulge-dominated vs. total in each magnitude bin.

TABLE 1

FUV and NUV LF Schechter Fit For Various Subsamples

\begin{tabular}{|c|c|c|c|c|}
\hline Subsample & $\begin{array}{c}\log \phi_{\star}\left({ }^{0.1} M^{\star}\right) \\
\left(\mathrm{Mpc}^{-3}\right)\end{array}$ & ${ }^{0.1} M^{\star}$ & $\alpha$ & $\begin{array}{c}\log \rho_{\nu} \\
\left(\mathrm{erg} \mathrm{s}^{-1} \mathrm{Mpc}^{-3}\right)\end{array}$ \\
\hline \multicolumn{5}{|c|}{ FUV } \\
\hline 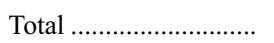 & $-2.08 \pm 0.02$ & $-17.83 \pm 0.04$ & $-1.06 \pm 0.04$ & 25.75 \\
\hline \multirow[t]{2}{*}{$n<2.4 \ldots \ldots \ldots \ldots \ldots \ldots \ldots$} & $-2.12 \pm 0.02$ & $-17.70 \pm 0.04$ & $-1.02 \pm 0.05$ & 25.65 \\
\hline & $-3.03 \pm 0.03$ & $-18.09 \pm 0.06$ & $-0.94 \pm 0.07$ & 24.88 \\
\hline \multirow[t]{2}{*}{$\log \mu_{\star}<8.5 \ldots \ldots \ldots \ldots \ldots$} & $-2.17 \pm 0.02$ & $-17.78 \pm 0.04$ & $-1.08 \pm 0.05$ & 25.64 \\
\hline & $-2.79 \pm 0.02$ & $-17.94 \pm 0.05$ & $-0.91 \pm 0.05$ & 25.04 \\
\hline \multirow[t]{2}{*}{$q_{25}<0.6 \ldots \ldots \ldots \ldots \ldots$} & $-2.31 \pm 0.04$ & $-17.37 \pm 0.07$ & $-1.06 \pm 0.09$ & 25.34 \\
\hline & $-2.27 \pm 0.02$ & $-17.86 \pm 0.04$ & $-0.87 \pm 0.05$ & 25.53 \\
\hline \multicolumn{5}{|c|}{ NUV } \\
\hline 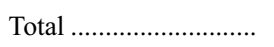 & $-2.27 \pm 0.02$ & $-18.44 \pm 0.03$ & $-1.21 \pm 0.03$ & 25.88 \\
\hline \multirow{2}{*}{$n<2.4$} & $-2.33 \pm 0.02$ & $-18.34 \pm 0.04$ & $-1.20 \pm 0.04$ & 25.77 \\
\hline & $-3.03 \pm 0.03$ & $-18.49 \pm 0.04$ & $-0.99 \pm 0.04$ & 25.06 \\
\hline \multirow[t]{2}{*}{$\log \mu_{\star}<8.5 \ldots \ldots \ldots \ldots$} & $-2.37 \pm 0.02$ & $-18.36 \pm 0.04$ & $-1.23 \pm 0.04$ & 25.75 \\
\hline & $-2.86 \pm 0.02$ & $-18.51 \pm 0.03$ & $-1.01 \pm 0.03$ & 25.24 \\
\hline \multirow{2}{*}{$q_{25}<0.6 \ldots \ldots \ldots \ldots \ldots \ldots$} & $-2.47 \pm 0.03$ & $-17.91 \pm 0.06$ & $-1.18 \pm 0.06$ & 25.45 \\
\hline & $-2.42 \pm 0.02$ & $-18.43 \pm 0.03$ & $-1.00 \pm 0.04$ & 25.65 \\
\hline
\end{tabular}



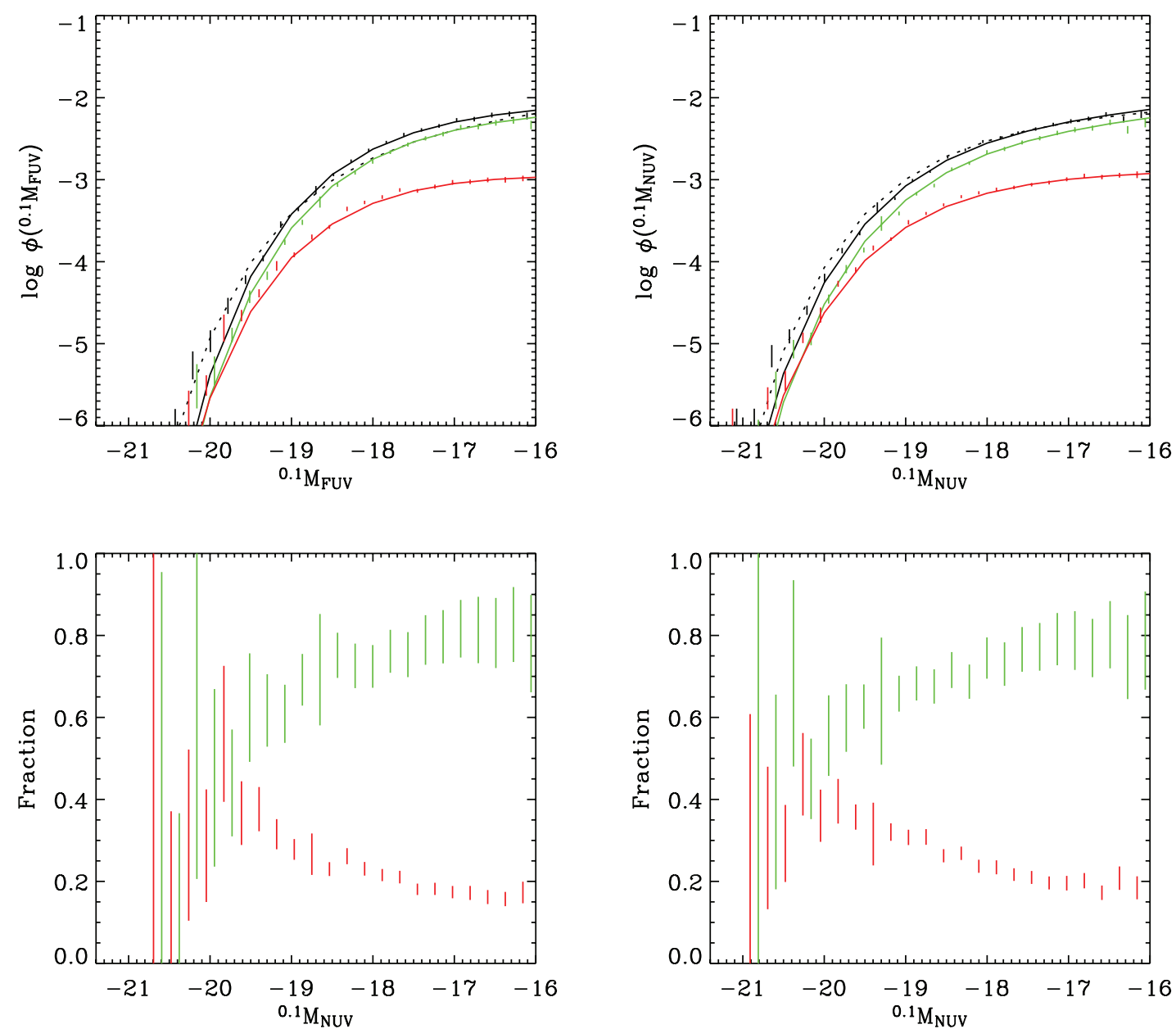

FIG. 4.-Top: FUV and NUV luminosity function for complete sample (black) and low and high stellar mass surface density subsamples split by log $\mu_{\star}: \log \mu_{\star}<8.5$ (green) and $\log \mu_{\star}>8.5$ (red). Units of $\phi$ are in $\mathrm{Mpc}^{-3} \mathrm{mag}^{-1}$. The dotted curve is from the Wyder et al. (2005) and Treyer et al. (2005) LF. Bottom: Relative fraction $\left(1 / V_{\max }\right.$-weighted) of low and high stellar mass surface density vs. total.

are consistent with those of Dahlen et al. (2007) based on data at higher redshift from GOODS, as well as with Menanteau et al. (2005).

We can nevertheless ask, How might we physically interpret this result? If the SFR is considered to be proportional to the UV (i.e., we assume no dust correction is needed), then the luminosity functions suggest that galaxies with the highest SFRs show a higher prevalence of bulge-dominated galaxies. This result is in agreement with Brinchmann et al. (2004) and Salim et al. (2005), which both reported a population of high star formation rate, high concentration galaxies. However, some bulge-dominated systems are likely to be massive, so while SFR might be high, SFR $/ M_{\star}$ may vary considerably. If many UV-luminous galaxies are dusty, then they are also likely to span a wide range of (dust-corrected) SFRs. In fact, as Hoopes et al. (2007) report, the most UV luminous galaxies are known to be a diverse population, containing disks and compact systems of a wide range of stellar masses and dust attenuations. It is unclear from our luminosity functions how these luminous disk and bulge-dominated galaxies will be distributed once a dust correction is made.

We have highlighted two crucial pieces of information that are needed to interpret the UV luminosity plots: the dependence on stellar mass, and the application of a dust correction that would allow us to interpret our results in terms of the galaxy's star formation history. (A third factor, inclination, is discussed further in the Appendix). To achieve this, we require reliable dust-attenuation corrections and SFR and $M_{\star} / L$ conversions. Below we describe our dust-attenuation corrections and SFR calibration, which we then apply to the color-magnitude distribution to construct a $\mathrm{SFR} / M_{\star}$ vs. $M_{\star}$ diagram. We use this more complete description of the galaxy population to study various scaling relations. Later in the paper we use this description to study the disk and bulgedominated populations separately.

\subsection{Star Formation Rates, Dust Attenuation, $\mathrm{SFR} / M_{\star}$, and $\Sigma_{\mathrm{SFR}}$}

In order to derive star formation rates from our UV-optical measures, we need to account for any dust attenuation which may cause us to underestimate the intrinsic luminosity of the galaxy. The dust-attenuation correction at any wavelength is nontrivial, since it is not simply a line-of-sight extinction correction, but reflects assumptions about the geometry of the emitting regions and the surrounding dust. Several recent investigations have explored how this dust geometry might be linked to galaxy morphology (e.g., Pierini et al. 2004; Dale et al. 2007; Zheng et al. 2007).

We hope to obtain reliable star formation rates for disk and bulge-dominated galaxies, and the latter population, with its lower SFR $/ M_{\star}$ and intrinsically red spectrum, presents a considerable challenge in this regard. Dust-attenuation measures derived from the UV using the relation determined for starburst or star-forming galaxies are unlikely to provide an accurate value 
TABLE 2

Measured and Derived Properties along the Star-forming Sequence

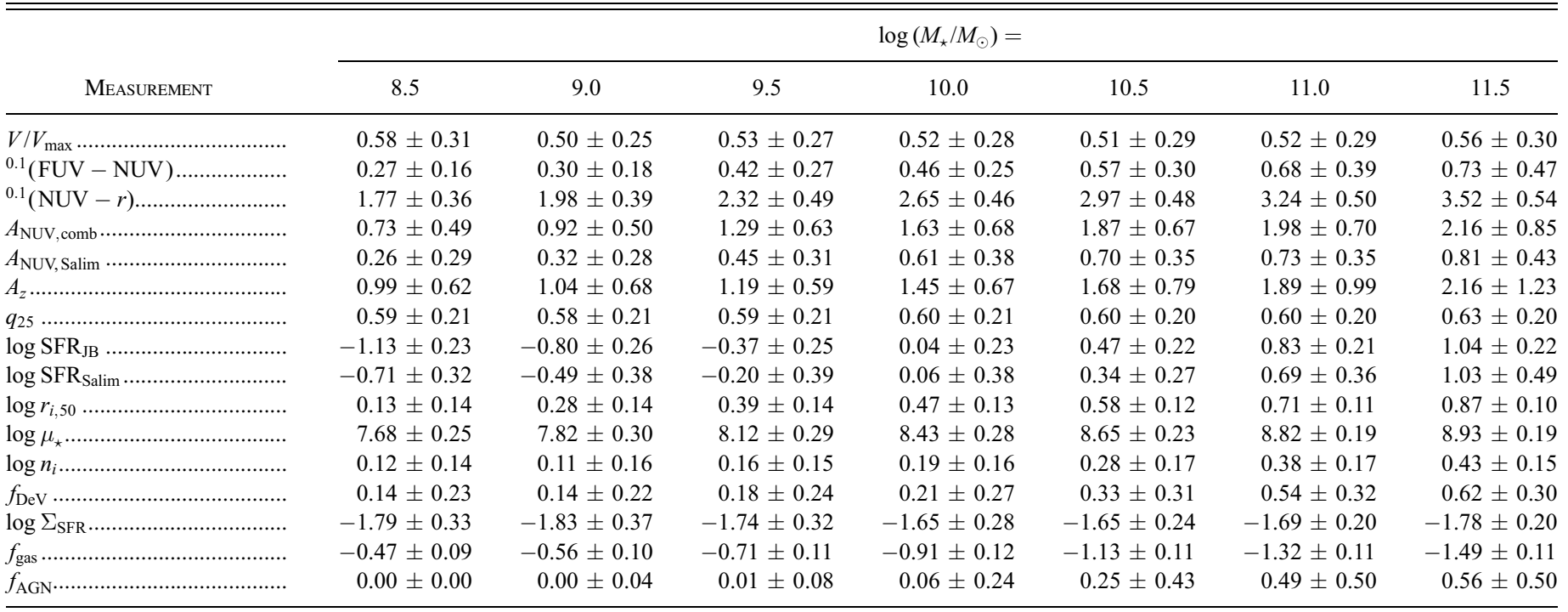

Note.-Values for $1 \sigma$ confidence interval half-width are given for each entry. Units for SFR in $M_{\odot} \mathrm{yr}^{-1}, r_{i, 50}$ in kpc, $\mu$ in $M_{\odot} \mathrm{kpc}^{-2}$, and $\Sigma_{\mathrm{SFR}}$ in $M_{\odot} \mathrm{yr}^{-1} \mathrm{kpc}^{-2}$.

(e.g., Bell 2002; Kong et al. 2004). Fiber emission-line measures (e.g., Balmer decrement) face similar problems, in addition to requiring aperture corrections, and are often not available at high $\mathrm{S} / \mathrm{N}$ for bulge-dominated galaxies (Brinchmann et al. 2004). UV-optical SED fitting, such as that adopted by S07, relies heavily on the calibration and accuracy of the models of young and old stellar populations and the dust-attenuation law, which are highly uncertain for evolved systems. Even the IR-to-UV flux ratio (unavailable for this work), often hailed as the most reliable dust-attenuation measure, remains difficult to interpret for evolved galaxies because of the uncertainty in determining which population (young or old stars, AGN) is contributing to the heating of the dust (e.g., Johnson et al. 2007).

Here we adopt a hybrid approach, which attempts to combine measures that work effectively on different subsets of the galaxy distribution. Johnson et al. $(2006,2007)$ present an IR-calibrated measure of the FUVattenuation $\left(A_{\mathrm{IRX}}\right)$ for a sample of 1000 SDSS galaxies, based on UV-optical colors and $D_{n}(4000)$ which correlates with star formation history,

$$
A_{\mathrm{IRX}}=0.81-1.32 x+1.07 y-0.81 x y
$$

where $x={ }^{0.1}(\mathrm{NUV}-z)-2$ and $y=D_{n}(4000)-1.25$ using coefficients taken from Table 2 in Johnson et al. (2007). The derivation of $A_{\text {IRX }}$ is most accurate for galaxies with $D_{n}(4000)<1.7$. Kauffmann et al. (2003a) provide an attenuation measure $A_{z}$ that is based on detailed model fits to the SDSS absorption-line spectrum and broadband SED, likely to be accurate for galaxies with higher $D_{n}(4000)$. Our combined fit provides a weighted mean of these two measures, using the measurement errors and published scatter for $A_{\mathrm{IRX}}$ and the $1 \sigma$ confidence intervals for $A_{z}$. Specifically, we combine them as:

$$
A_{\mathrm{NUV}, \mathrm{comb}}=\frac{A_{\mathrm{FUV}, \mathrm{IRX}} /\left(k_{\mathrm{FUV}} \sigma_{A_{\mathrm{FUV}, \mathrm{IRX}}}^{2}\right)+A_{z} /\left(k_{z} \sigma_{A_{z}}^{2}\right)}{1 /\left(k_{\mathrm{FUV}} \sigma_{A_{\mathrm{FUV}, \mathrm{IRX}}}\right)^{2}+1 /\left(k_{z} \sigma_{A_{z}}\right)^{2}},
$$

where we have used the Calzetti et al. (2000) attenuation curve to derive $k_{\mathrm{FUV}}=A_{\mathrm{NUV}, c} / A_{\mathrm{FUV}, c}=0.81$ and $k_{z}=A_{\mathrm{NUV}, c} / A_{z, c}=3.21$.
We show in Figure 5 a comparison between our derived dust attenuation and other measures, all converted to $A_{\mathrm{NUV}}$ using the Calzetti et al. (2000) attenuation curve and plotted as a function of $D_{n}(4000)$ : $A_{\mathrm{NUV}, \mathrm{IR}}$ from Johnson et al. (2007), $A_{\mathrm{NUV}, z}$ from Kauffmann et al. (2003a), $A_{\mathrm{NUV}}$,Salim from S07, and $A_{\mathrm{NUV}, \mathrm{BETA}}$ derived using the IRX- $\beta$ relation obtained by Seibert et al. (2005). The first two plots compare quantities used to derive $A_{\mathrm{NUV}}$,comb and illustrate the range over which each attenuation measure is given higher weight, with $A_{z}$ being the dominant measure for older galaxies and $A_{\mathrm{NUV}}$,IR for younger galaxies. As expected, $A_{\mathrm{NUV}, \mathrm{IR}}$ is systematically higher for older galaxies (Johnson et al. 2007). Our hybrid attenuation measure shows relatively good agreement $\left(\left|\overline{\Delta A_{\mathrm{NUV}}}\right|<\right.$ 0.5) with S07 and Seibert et al. (2005), albeit with large scatter throughout and lower values for the oldest galaxies. Johnson et al. (2007) explore the scatter and systematic differences between these measures and others, including the Balmer decrement (see also Paper I), which are beyond the scope of this work. We have repeated almost all analyses in this paper using each of the different measures, and although there are notable systematic differences, our overall results and conclusions do not change significantly.

We applied this dust-attenuation correction to our NUV luminosities to obtain an "intrinsic" NUV luminosity. We then used these values to determine a star formation rate using the formula

$$
\operatorname{SFR}\left(M_{\odot} \mathrm{yr}^{-1}\right)=10^{-28.165} L_{\nu}\left(\mathrm{erg} \mathrm{s}^{-1} \mathrm{~Hz}^{-1}\right)
$$

derived by S07 assuming a Kroupa IMF and a continuous recent (100-300 Myr) star formation history, which makes these star formation rates directly comparable to those in that work and most other recent determinations. Note that for a standard Salpeter IMF (between 0.1 and $100 M_{\odot}$ ), star formation rates would be a factor of $\sim 1.5$ higher.

Figure 6 compares our derived SFRs with those of S07 and Brinchmann et al. (2004). Again, we find relatively good agreement with S07 except at the highest stellar masses and oldest galaxies, where our star formation rates tend to be slightly higher. A wider systematic trend is observed with the $\mathrm{H} \alpha$ [and color$\left.D_{n}(4000)\right]$ derived star formation rates from Brinchmann et al. 

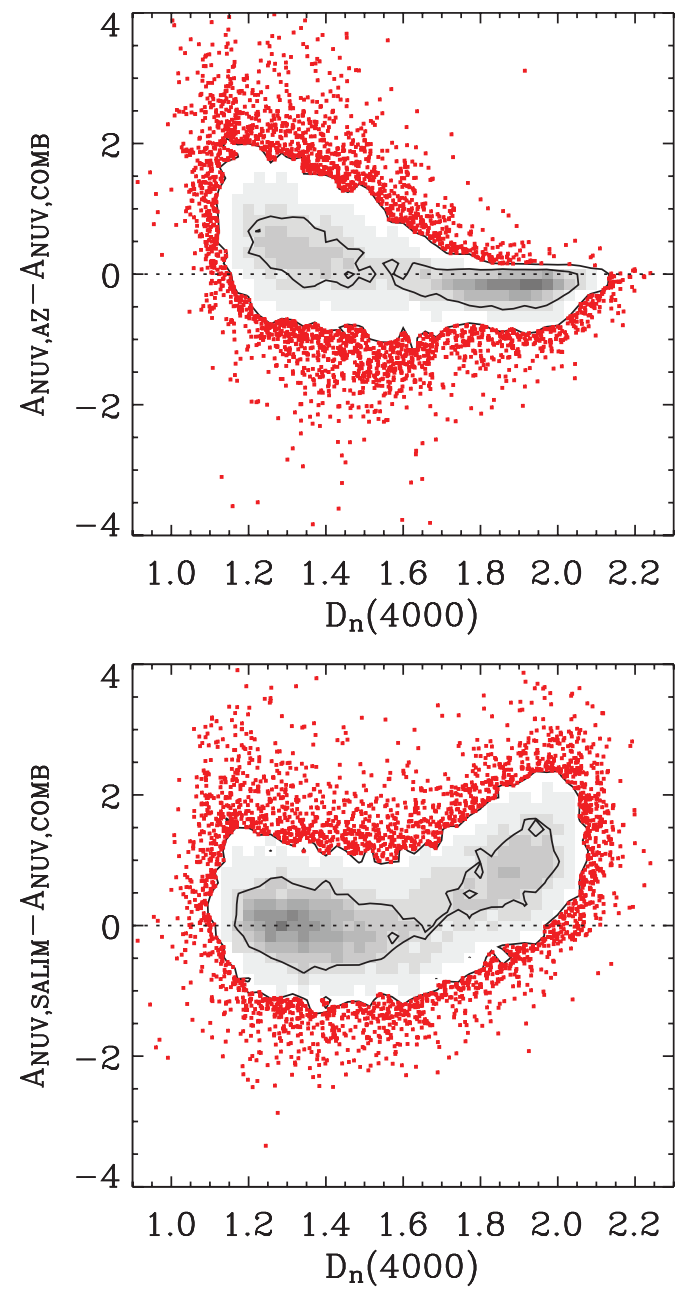
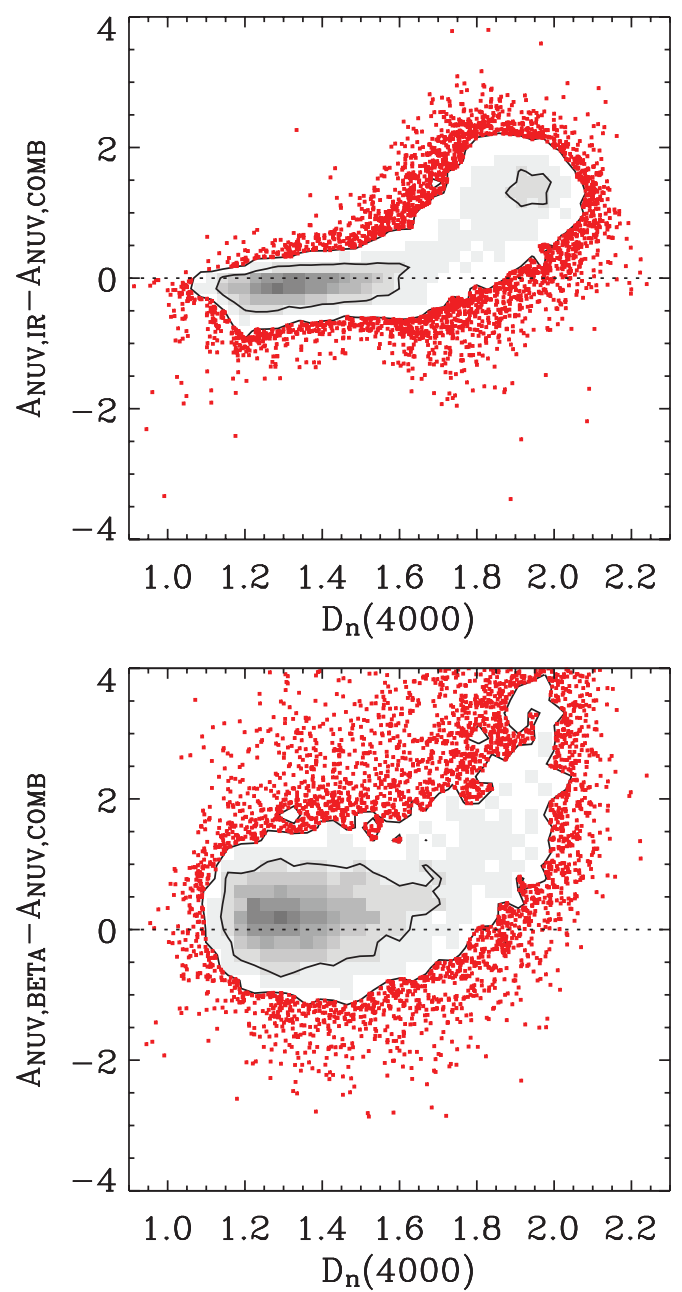

FIG. 5.-Comparison of different dust-attenuation measures ( $\left.A_{\mathrm{NUV}}\right)$ with the "combined" measure used in this paper. Top left: $A_{\mathrm{NUV}}$ derived using $A_{z}$ from Kauffmann et al. (2003c). Top right: $A_{\mathrm{NUV}}$ derived using empirically derived attenuation measure from Johnson et al. (2007). Bottom left: $A_{\mathrm{NUV}}$ derived by S07. Bottom right: $A_{\mathrm{NUV}}$ using the Seibert et al. (2005) IRX- $\beta$ relation.

(2004), with our values significantly lower for the most massive and oldest galaxies. Galaxies not classified as "star-forming" in Brinchmann et al. (2004) have SFRs derived using $D_{n}(4000)$, optical colors, and aperture corrections. UV-derived SFRs have been shown to possess a higher dynamic range and are therefore applicable to a broader range of galaxy types (see S07 for an extensive discussion of these differences.) As with the attenuation measures discussed above, we find that despite the differences, our overall conclusions are largely independent of the choice of SFR measure used.

All specific star formation rates $\left(\mathrm{SFR} / M_{\star}\right)$ in this paper are calculated using the NUV-derived star formation rate, $\mathrm{SFR}_{\mathrm{comb}}$, and the MPA/JHU stellar mass, $M_{\star}$. Global star formation rate surface densities $\left(\Sigma_{\mathrm{SFR}}\right)$ were calculated using $\mathrm{SFR}_{\text {comb }}$ and the $u$-band half light radius, $R_{u, 50}$,

$$
\Sigma_{\mathrm{SFR}}=\frac{0.5 \mathrm{SFR}}{\pi R_{u, 50}^{2}} .
$$

Our definition differs somewhat from other studies which calculate a global $\Sigma_{\mathrm{SFR}}$, out to the edge of the optical disk (e.g., Martin \& Kennicutt 2001). For the purposes of our analysis, our definition is sufficiently similar that we can neglect this difference, but will return to it in future work.

\subsection{The Color-Magnitude and SFR $/ M_{\star}-M_{\star}$ Distribution}

Figure 7 replicates the final result from Paper I, which we reproduce here in slightly modified form using the analysis described above. In the left panel of Figure 7 we plot the $1 / V_{\max }$-weighted distribution of galaxies as a function of ${ }^{0.1}(\mathrm{NUV}-r)$ vs. ${ }^{0.1} M_{r}$ calculated as described in the previous section. In the central panel we plot ${ }^{0.1}(\mathrm{NUV}-r)_{\text {cor }}$ vs. ${ }^{0.1} M_{r \text {,cor }}$, where we have applied our derived dust attenuation corrections $A_{\mathrm{NUV}, \text { comb }}$ to the measurement in each band. Finally, in the right panel we plot the specific star formation rate SFR $/ M_{\star}$ vs. $M_{\star}$ based on the quantities derived from our dust-corrected luminosities. Note that in this paper we adhere to an adopted terminology in which "blue sequence" refers to the locus of blue galaxies in the uncorrected or dust-corrected color magnitude diagram, and "SF sequence" refers to the locus of star-forming galaxies in the SFR $/ M_{\star}$ vs. $M_{\star}$ diagram.

As described in Paper I, the blue sequence follows a shallow slope in the UV-optical color-magnitude diagram, with a reddening trend toward higher stellar mass galaxies. Above a stellar mass of $10^{10} M_{\odot}$ the sharpness of the sequence decreases and a wide spread of colors are seen, extending up to the red limit of our sample. We have overplotted the curve following the peak of the blue sequence derived in Paper I. In the middle plot, the corrected blue sequence displays a much shallower slope with 

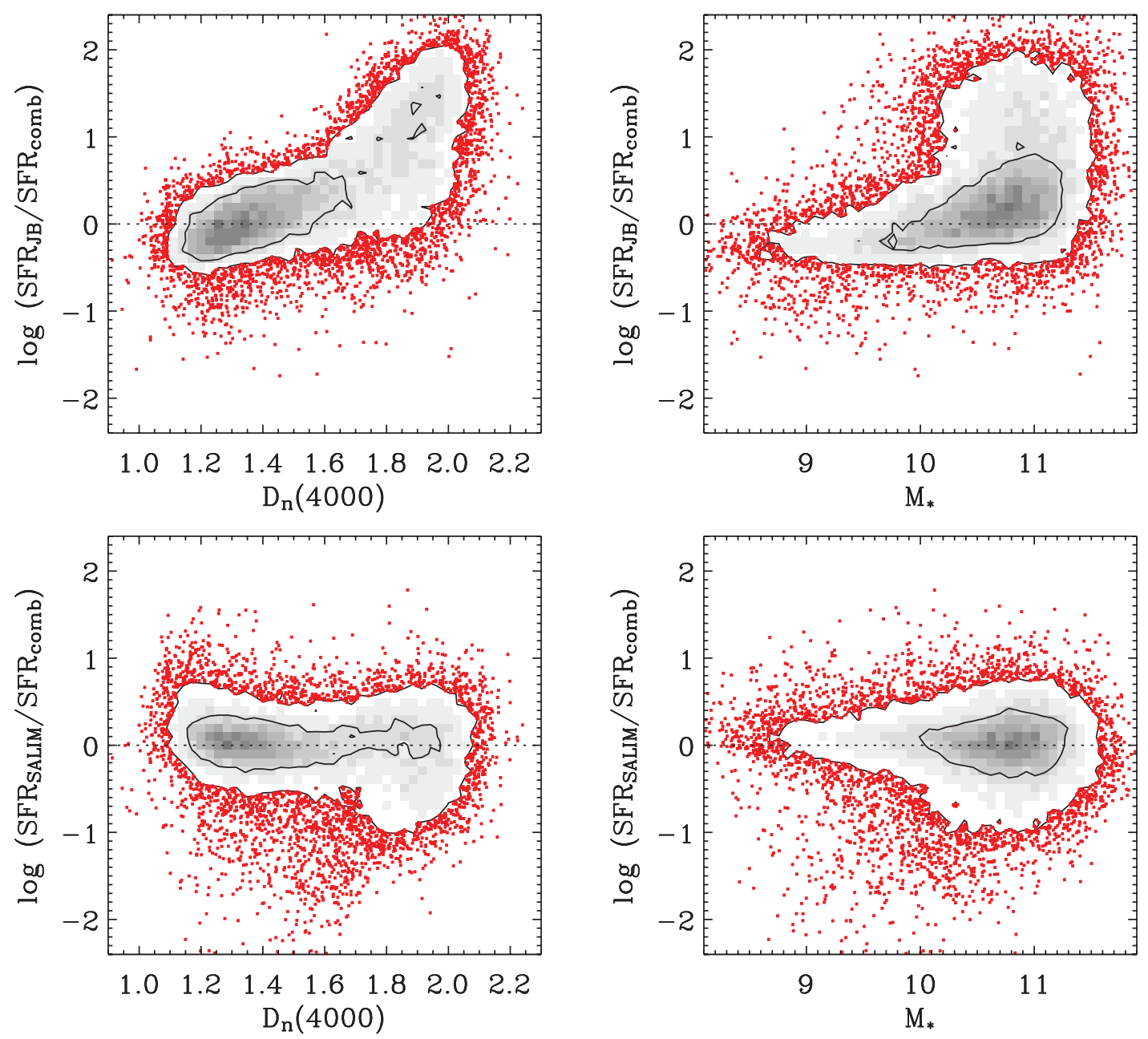

FIG. 6.- Comparison of different SFR measures with the one used in this paper, plotted vs. $D_{n}(4000)$ and $M_{\star}$. Top: SFRs from Brinchmann et al. (2004). Bottom: SFRs from S07.
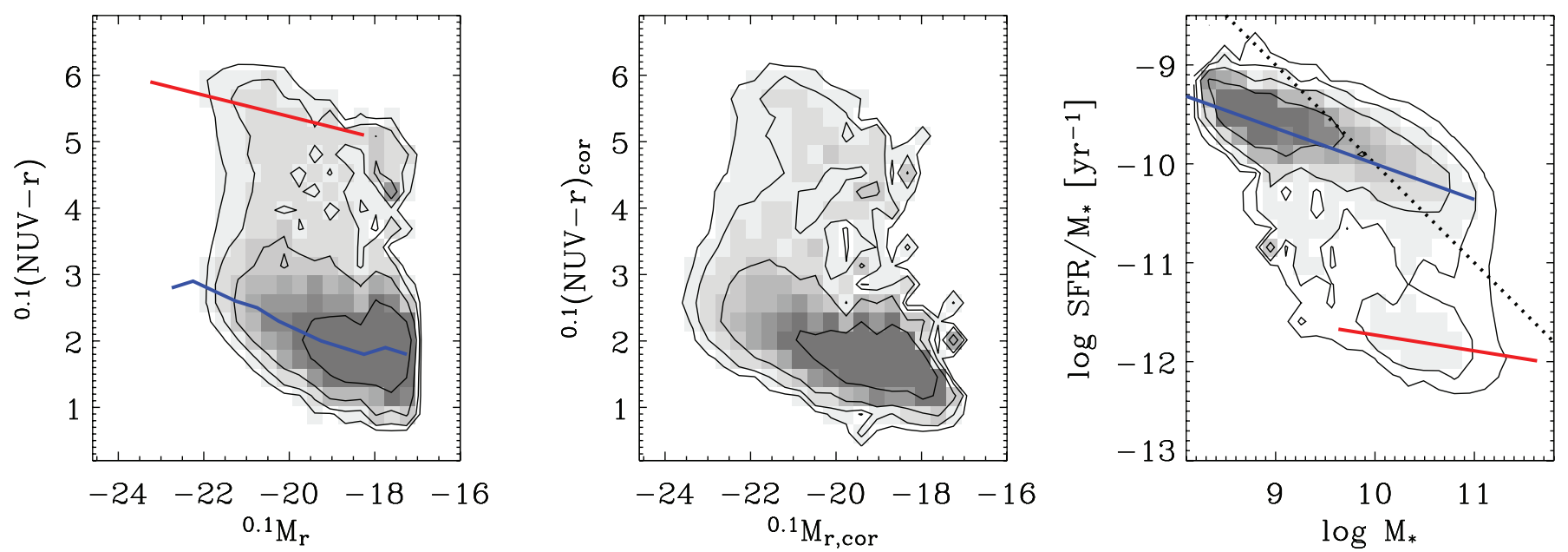

FIG. 7.-Bivariate distribution (1/V $V_{\max }$-weighted) of galaxies as a function of color vs. magnitude and SFR $/ M_{\star}$ vs. $M_{\star}$. Left: $^{0.1}$ (NUV $\left.-r\right)$ color vs. ${ }^{0.1} M_{r}$ using $K$-corrected absolute magnitudes. No dust-attenuation correction has been applied. Blue and red solid lines trace the ridge line of the blue and red sequences, taken from Paper I. Center: Dust attenuation corrections have been applied to both the NUV and $r$ absolute magnitudes. Right: SFR $/ M_{\star}$ vs. $M_{\star}$. The blue solid line shows the starforming sequence fit from $\mathrm{S} 07$, and the red line shows approximate position of non-star-forming sequence on this diagram. Contours enclose $38 \%, 68 \%, 87 \%$, and $95 \%$ of the distribution. (See text for details). Dotted line: $\mathrm{SFR}=1 M_{\odot} \mathrm{yr}^{-1}$. 

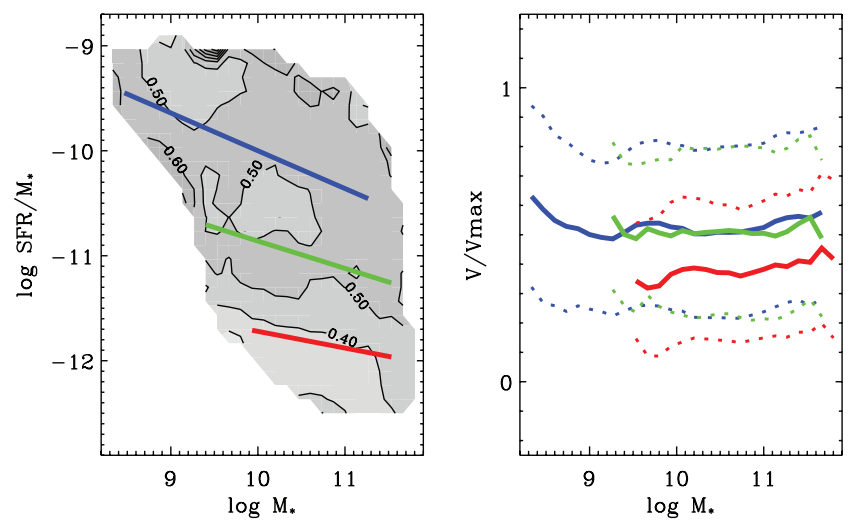

FIG. 8.-Left: Mean of $V / V_{\max }$ as a function of SFR $/ M_{\star}$ vs. $\log M_{\star}$. The blue solid line shows SF sequence ridge line from S07, and the red solid line for non$\mathrm{SF}$ galaxies is based on red sequence fit from Paper I. The green solid line represents residual-SF galaxies ("green valley"), following the geometric mean of $\mathrm{SF}$ and non-SF sequences. Right: Weighted mean and $\pm 1 \sigma$ distribution width on $V / V_{\max }$ along blue, green, and red lines.

stellar mass, and a more sharply defined "peak." On the right, this sequence tilts again, a result of the conversion of dustcorrected $r$-band luminosity to stellar mass, which varies across the sequence (with $M_{\star} / L$ increasing with stellar mass). We overplot the SF sequence with a line defined by ${ }^{14}$

$$
\log \mathrm{SFR} / M_{\star}=-0.36 \log M_{\star}-6.4 .
$$

We show below that this line closely follows the ridge line (peak) of the star forming distribution, even to high stellar masses; therefore, we do not attempt to provide a more rigorous definition. The line is also consistent with a $z \sim 0.1$ extrapolation of the one derived by Noeske et al. (2007a, 2007b) over a range of redshifts $(0.3<z<1.1)$, in both slope and normalization.

We overplot a trend line for non-star-forming (non-SF) galaxies in the SFR $/ M_{\star}$ vs. $M_{\star}$ diagram, but caution the reader that unlike the SF sequence, this locus is likely to misrepresent the true distribution of non-SF galaxies. In fact, the SFR $/ M_{\star}$ of galaxies in this part of the diagram is best taken as an upper limit; in practice it remains extremely difficult to probe star formation at levels below $\log \mathrm{SFR} / M_{\star} \sim-12$. Many evolved galaxies possess weak UV emission that is probably not associated with recent star formation (e.g., Rich et al. 2005; Yi et al. 2005). This UV light from evolved stars is most likely responsible for the red sequence locus in the color-magnitude diagram. While some authors have suggested that an AGN may contribute a fraction of the NUV luminosity in galaxies with reduced levels of star formation (e.g., Agüeros et al. 2005; Tremonti et al. 2007), emission-line diagnostics suggest that this is unlikely to be significant for the majority of galaxies in our sample.

In the next series of figures we also highlight the location of intermediate ("green valley") galaxies with a line defined by the geometric mean of the blue and the red. The green valley is meant to identify galaxies that lie between the blue and red sequences on the color magnitude diagram, although as we discuss in the next section this population is quite heterogeneous (e.g., Johnson et al. 2007). Considering the above caveats for the (log-log) SFR/ $M_{\star}$ vs. $M_{\star}$ diagram, describing this region as a "valley" may not be accurate - galaxies may not actually show a double peaked

\footnotetext{
14 Taken from an earlier analysis by Salim et al. prior to final result published in S07 $\left(\log \mathrm{SFR} / M_{\star}=-0.35 \log M_{\star}-6.33\right)$. The difference is small, with little impact on overall conclusions.
}
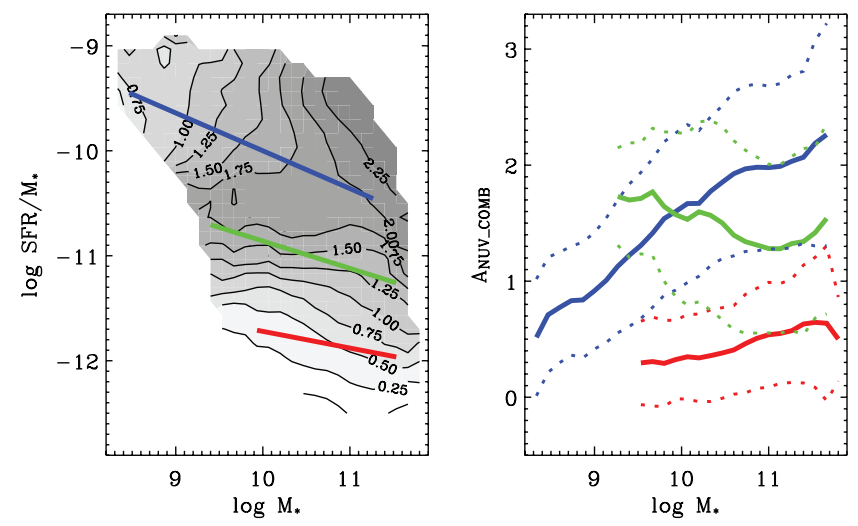

FIG. 9.-Left: Weighted mean of NUV-band attenuation, $A_{\mathrm{NUV}, \mathrm{comb}}$. Right: Weighted mean and $\pm 1 \sigma$ distribution width for $A_{\mathrm{NUV}}$,comb along similarly colored curves in above plots. (See caption of Fig. 8 for explanation.)

distribution in SFR $/ M_{\star}$ at fixed stellar mass. ${ }^{15}$ The "green valley" is most physically meaningful in describing a residual star-forming population (e.g., Yi et al. 2005) in the dust-corrected color-magnitude diagram shown as the middle plot of Figure 7 and discussed extensively in Paper III. In reference to the SFR $/ M_{\star}$ vs. $M_{\star}$ diagram, we refer to these as "residual-SF" galaxies with $\log \mathrm{SFR} / M_{\star} \sim-11$. At their present rate, residual-SF galaxies will form an additional $\sim 1 \%-10 \%$ in stellar mass over a gigayear.

\subsection{Trends along the SF, Residual-SF, and Non-SF "Sequences"}

The identification of the SF sequence presents a unique opportunity to study the properties of the dominant star-forming population independently of any color, spectroscopic, or morphologically defined selection criteria. In this section we focus on trends along the SF sequence, and we also compare the properties of SF, residual-SF and non-SF galaxies. To accomplish this, we calculated the $1 / V_{\max }$-weighted mean and standard deviation of a given measure (linear or logarithmic, as indicated) in bins of SFR $/ M_{\star}$ vs. $M_{\star}$. Bins were spaced by 0.13 dex in both $\mathrm{SFR} / M_{\star}$ and $M_{\star}$, and we only considered bins containing more than 20 galaxies. For each two-dimensional distribution we then measured weighted means (and standard deviations) vs. $M_{\star}$ along the SF, residual-SF, and non-SF sequences defined by the linear relations described above. Results along the SF sequence for a number of measured and derived properties are given in Table 2. Figures 8-16 show the two-dimensional and one-dimensional distribution of weighted means of several key measurements and physical properties.

Since we are considering trends across the entire galaxy population, we first investigate whether any region of parameter space suffers from sample incompleteness which might influence our subsequent analysis. Figure 8 shows the (unweighted) mean distribution of $V / V_{\max }$ for which we expect a value of 0.5 . We find deviations from this value only for the lowest stellar masses and lowest SFR $/ M_{\star}$, neither of which will significantly impact our results. Another crucial component of our analysis is the dust-attenuation correction. We plot $A_{\mathrm{NUV}, \text { comb }}$ in Figure 9 which shows a clear trend toward increasing $A_{\mathrm{NUV}}$,comb along the SF sequence toward higher $M_{\star}$. The highest dust attenuations are found for galaxies with the highest SFR $/ M_{\star}$ and $M_{\star}$,

\footnotetext{
15 However, it is possible that galaxies of a given $M_{\star}$ might show a low level of star formation fed by gas associated with stellar mass loss (Knapp et al. 1992) and/or cooling flows (Fabian et al. 1982), or other phenomena (Mathews \& Brighenti 2003).
} 

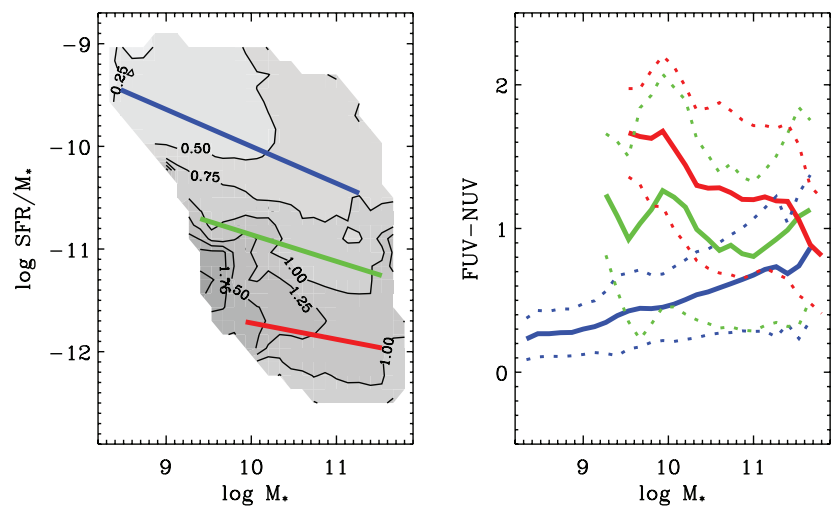

FIG. 10.-Left: Weighted mean of rest-frame FUV-NUV color. Right: Weighted mean and $\pm 1 \sigma$ distribution width for FUV-NUV along similarly colored curves in above plots. (See caption of Fig. 8 for explanation.)

in the top right of the diagram. Residual-SF and non-SF galaxies show lower attenuations for the same $M_{\star}$. We can compare these results with the average FUV - NUV colors shown in Figure 10 which has been shown by Meurer et al. (1999), Seibert et al. (2005), and Johnson et al. (2007) to correlate with dust attenuation in galaxies with ongoing star formation. Not surprisingly, we find a similar trend along the SF sequence with $\mathrm{FUV}-\mathrm{NUV} \simeq A_{\mathrm{NUV}, \mathrm{comb}} / 3.1$, in almost exact agreement with our expectation based on Seibert et al. (2005) and Calzetti et al. (2000).

Observed axis ratios are known to correlate with galaxy inclination (Binney \& de Vaucouleurs 1981). In Figure 11 we use the isophotal axis ratio $q_{25}=b_{25, r} / a_{25, r}$ to study the distribution of inclination across the galaxy population. While the total variation is small, we do find a minimum at SFR $/ M_{\star}$ slightly below the SF sequence. A color-derived attenuation correction will fail to fully remove inclination effects at optical depths $\tau>1$, e.g., saturating in edge-on galaxies. One possible interpretation is that our dust-attenuation correction has not fully removed inclination-dependent effects. However, an alternative is that there is a prevalence of disk-dominated galaxies (which show a wider distribution of axis ratios than irregular or bulgedominated galaxies) in that part of the SFR $/ M_{\star}$ vs. $M_{\star}$ diagram. We consider this point further in the Appendix.

The next set of plots shows the variation of structural parameters along the SF sequence, highlighting four parameters: $r_{50}$ and $\log \mu_{\star}$, which are related to the underlying stellar mass distribution, $\Sigma_{\mathrm{SFR}}$, a measure of the star formation rate surface
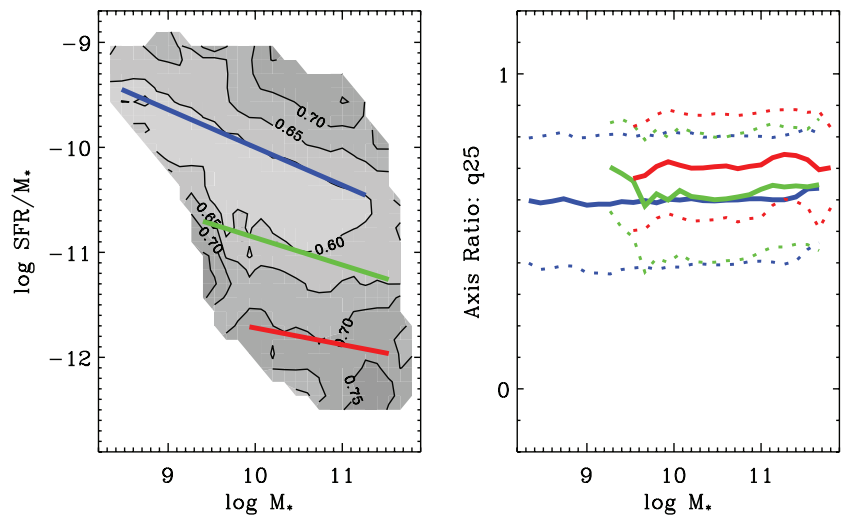

FIg. 11.-Left: Weighted mean of axis ratio $q_{25}$, used as a measure of inclination. Right: Weighted mean and $\pm 1 \sigma$ distribution width for axis ratio $q_{25}$ along similarly colored curves in above plots. (See caption of Fig. 8 for explanation.)
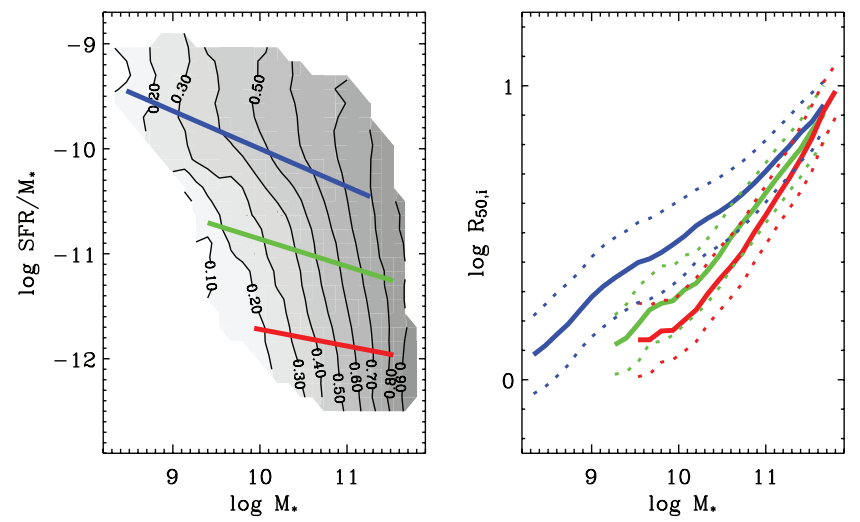

FIG. 12.-Left: Weighted mean of logarithm of the $i$-band half-light $(50 \%)$ radius, $\log r_{50, i}$ (physical, in kpc). Right: Weighted mean and $\pm 1 \sigma$ distribution width for $\log r_{50, i}$ along similarly colored curves in above plots. (See caption of Fig. 8 for explanation.)

density, and the Sersic index $n_{i}$, which provides an indication of the ratio of bulge-to-total light. Figure 12 shows that the $i$-band half-light radius predominantly increases with $M_{\star}$. Shen et al. (2003) derived the stellar mass-radius relation for galaxies from SDSS and found for disk galaxies a dependence of $r \propto M_{\star}^{0.15}$ for $\log M_{\star}<10.5$ and $r \propto M_{\star}^{0.4}$ for $\log M_{\star}>10.5$. A fit to the relation along the SF sequence results in $r \propto M_{\star}^{0.22}$, with a dependence that steepens to $r \propto M_{\star}^{0.3}$ at higher stellar masses. Residual-SF and non-SF sequence galaxies display an even steeper slope for the $M_{\star}-r_{50}$ relation. Complementary trends are found for $\log \mu_{\star}$, shown in Figure 13. Surface mass density $\log \mu_{\star}$ varies smoothly along the SF sequence with $\mu \propto M_{\star}^{0.52}$ for $9<\log M_{\star}<11$. Over most of the stellar mass range, the residual-SF and non-SF galaxies show much less variation, saturating to nearly a constant value near $\log \mu_{\star}=9$. These results are similar to those found by Kauffmann et al. (2003b) for young, disk-dominated and old, bulge-dominated systems.

Unlike $\log \mu_{\star}$, the trend in the star formation rate surface density, $\Sigma_{\mathrm{SFR}}$, plotted in Figure 14, shows almost no variation along the SF sequence, with $\Sigma_{\mathrm{SFR}} \simeq 0.02 M_{\odot} \mathrm{yr}^{-1} \mathrm{kpc}^{-2}$, similar to the nearly constant FUV surface brightness, $I_{\mathrm{FUV}}$ vs. $M_{\star}$, noted in Hoopes et al. (2007). This value lies approximately at the middle of the range identified for "normal spirals" and "central regions of normal disks" by Kennicutt (1998b), although it is approximately 2 orders of magnitude below the typical $\Sigma_{\mathrm{SFR}}$ for starbursts (Kennicutt 1998b) and star-forming galaxies at $z=2$ (Erb et al. 2006). Given the scaling relations identified above, it
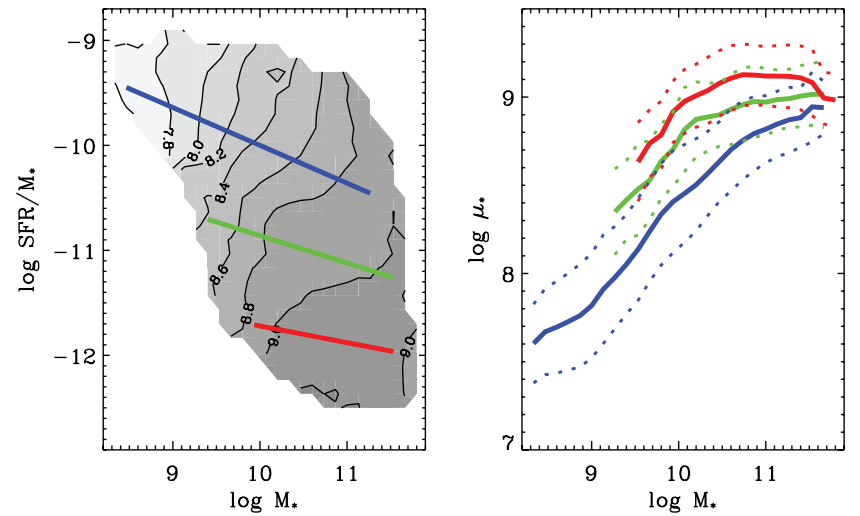

FIG. 13.-Left: Weighted mean of logarithm of the stellar mass surface density $\log \mu_{\star}$. Right: Weighted mean and $\pm 1 \sigma$ distribution width for $\log \mu_{\star}$ along similarly colored curves in above plots. (See caption of Fig. 8 for explanation.) 

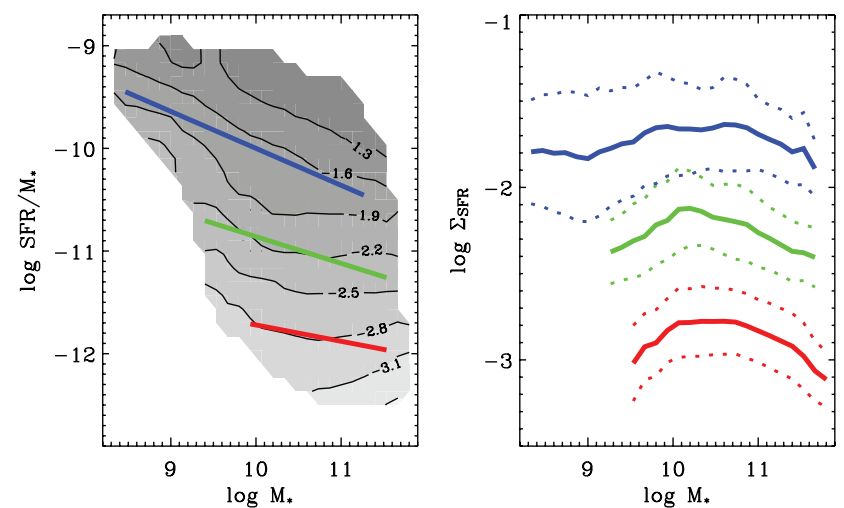

FIG. 14.-Left: Weighted mean of logarithm of the star formation rate surface density, $\log \Sigma_{\text {SFR }}$. Right: Weighted mean and $\pm 1 \sigma$ distribution width for $\log \Sigma_{\text {SFR }}$ along similarly colored curves in above plots. (See caption of Fig. 8 for explanation.)

is straightforward to derive a link between the ridge line of the SF sequence and the near constancy of $\Sigma_{\mathrm{SFR}}$ for these galaxies. If we assume that the ridge line has the approximate functional form

$$
\mathrm{SFR} \propto M_{\star}^{2 / 3},
$$

then combined with a stellar mass-radius relation

$$
r \propto M_{\star}^{1 / 3-\epsilon},
$$

we find that

$$
\Sigma_{\mathrm{SFR}} \propto M_{\star}^{2 \epsilon},
$$

or nearly constant for small $\epsilon$. This intriguing result would appear to suggest that the vast majority of star-forming galaxies are distributed such that their scaling relations are consistent with a single value for $\Sigma_{\text {SFR. }}$. It may also suggest a possible connection (via the global Schmidt law) to observations that show that disk-dominated and star-forming galaxies possess a narrow range in gas mass surface densities, despite displaying a much wider range of gas masses and radii (Haynes \& Giovanelli 1984; Roberts \& Haynes 1994; Kennicutt 1998a). Low surface brightness and starbursting galaxies will lie off of the SF sequence, but they are not a dominant population at $z \sim 0.1$.

This result also has interesting implications at higher redshift. Noeske et al. (2007a, 2007b) suggest that the SF sequence exists up to $z \sim 1$ with a similar slope vs. $M_{\star}$ but an evolving SFR intercept (normalization). Combined with the weak evolution of the stellar mass-radius relation out to $z \sim 1$ (e.g., Somerville et al. 2007), the fundamental change between the local and $z=1 \mathrm{SF}$ sequence is an increase in $\Sigma_{\mathrm{SFR}}$. In light of this, it is intriguing that a significant fraction of LIRGs at higher redshift appear to be star-forming disks with elevated SFRs (Zheng et al. 2007; Melbourne et al. 2005).

The highest $\Sigma_{\mathrm{SFR}}$, about 5 times higher (in the mean), are found in the high- $M_{\star}$, high SFR $/ M_{\star}$ region, which also shows the highest mean attenuations. This extreme part of the diagram is occupied by ultralumininous infrared galaxies (ULIRGs) and ultraviolet luminous galaxies (UVLGs; Heckman et al. 2005; Hoopes et al. 2007), although these populations are rare and will not contribute significantly to the weighted mean. The residual-SF and non-SF sequences show little variation of $\Sigma_{\mathrm{SFR}}$ with stellar mass, with levels of $\Sigma_{\text {SFR }}$ that are at most $1 / 3$ and 1/10 that of the SF sequence.
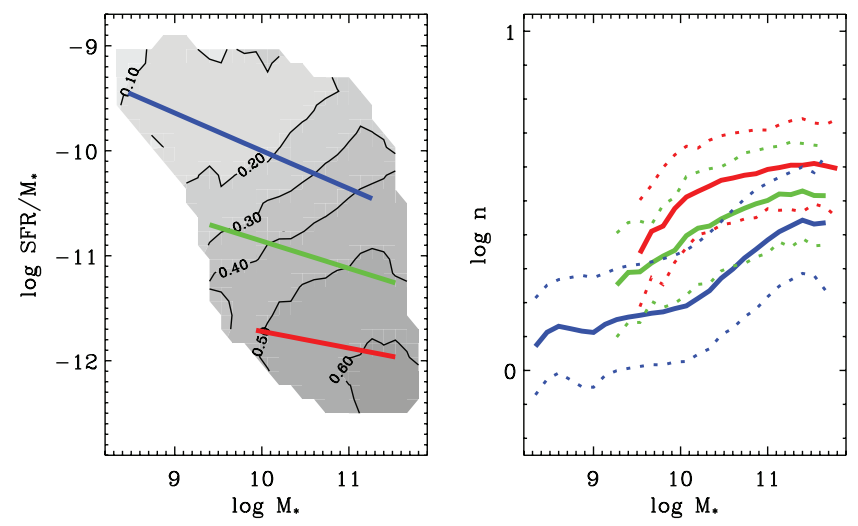

FIG. 15.-Left: Weighted mean of $\log$ arithm of the Sersic index, $\log n_{i}$. Right: Weighted mean and $\pm 1 \sigma$ distribution width for $\log n_{i}$ along similarly colored curves in above plots. (See caption of Fig. 8 for explanation.)

For the $\log$ arithm of the Sersic index, $\log n_{i}$, shown in Figure 15 , the mean profile along the SF sequence is relatively constant until stellar masses close to the "transition mass" $\left(\log M_{\star} \simeq 10.5\right)$ identified in Kauffmann et al. (2003a), where the Sersic index increases sharply. At any given $M_{\star}$, the mean Sersic index for residual-SF and non-SF galaxies is higher than on the SF sequence, with both showing a trend of increasing $\log n_{i}$ with increasing $M_{\star}$, particularly below the transition mass. It is clear from this plot that the SF sequence is not uniform in its structural properties, with a steady increase in bulge-dominated galaxies with increasing stellar mass, and significant scatter over the entire stellar mass range.

The detectability of an AGN has been shown to be correlated with the luminosity of the bulge component and the presence of gas within a galaxy (Kauffmann et al. 2003c). The fraction of AGNs [with $\log L(\mathrm{O} \text { III })_{\text {extcor }}>5$ ] as a function of SFR $/ M_{\star}$ and $M_{\star}$ is plotted in Figure 16. The trend along the SF sequence mimics the trend in Sersic index. In addition, along the residualSF line the AGN fraction remains high, suggesting that galaxies with small amounts of SF may still show the AGN phenomenon. The AGN fraction and its connection to residual-SF in "green valley" galaxies is discussed in more detail in Paper III and in $\mathrm{S} 07$.

We conclude this section by restating some key results:

1. In almost all respects, we find that the physical properties of galaxies vary smoothly (vs. $M_{\star}$ ) along the SF sequence. Although
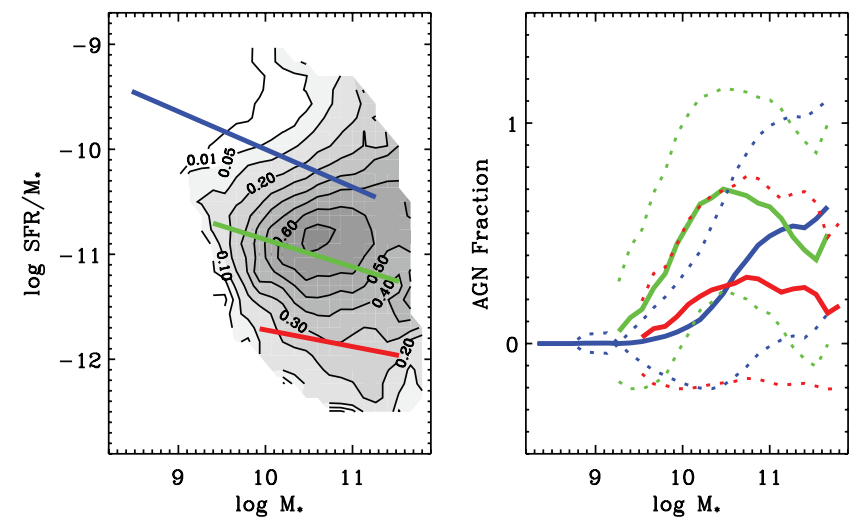

FIG. 16.-Left: Weighted mean of AGN fraction. Right: Weighted mean and $\pm 1 \sigma$ distribution width for AGN fraction along similarly colored curves in above plots. (See caption of Fig. 8 for explanation.) 

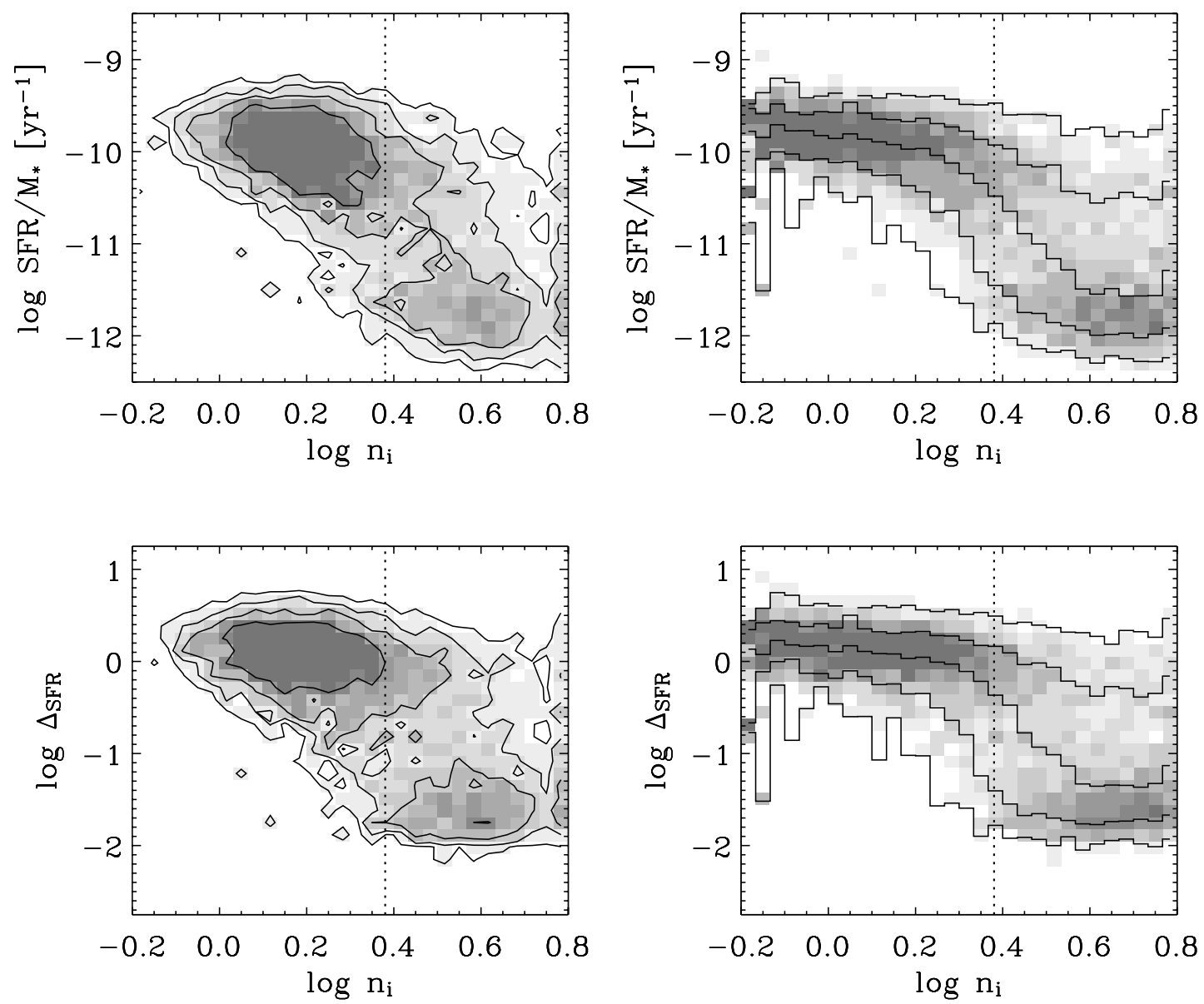

FIG. 17.- Specific star formation rate and "SFR excess" vs. galaxy light profile shape, plotting the bivariate distribution $\left(1 / V_{\max }\right.$-weighted) of galaxies as a function of $\mathrm{SFR} / M_{\star}($ top $)$ and $\Delta_{\mathrm{SFR}}$ (bottom) vs. log of $i$-band Sersic index $n_{i}$. Left: Contours enclose $38 \%, 68 \%, 87 \%$, and $95 \%$ of the distribution. Right: Conditional distribution in each $\log n_{i}$ bin. Quintiles represent $10 \%, 25 \%, 50 \%, 75 \%$, and $90 \%$ of the distribution in that bin.

we find known scaling relations for star-forming, disk and/or bulgedominated galaxies, we do recover some new and notable trends.

2. We find that $\Sigma_{\text {SFR }}$ remains nearly constant vs. $M_{\star}$ along the SF sequence, with $\Sigma_{\mathrm{SFR}}$ for non-SF galaxies least a factor of 10 lower on average.

3. At fixed $M_{\star}, r_{50}$ is higher and $\log \mu_{\star}$ and $\log n_{i}$ lower for the SF sequence compared to the residual-SF and non-SF sequences. This suggests that non-SF galaxies are not simply SF galaxies of the same stellar mass that have stopped forming stars-a result consistent with the observed correlation between SFH and structure. However, these differences between the SF and non-SF sequences are most profound near the transition mass. Above this stellar mass the structural properties of the population show considerably less scatter, despite the significant differences in $\mathrm{SFR} / M_{\star}$ and $\Sigma_{\mathrm{SFR}}$.

\section{ON THE RELATIONSHIP BETWEEN STAR FORMATION HISTORY AND STRUCTURE}

\subsection{SFR $/ M_{\star} v s . \log n_{i}$ and the SFR Excess, $\Delta_{\mathrm{SFR}} v s . \log n_{i}$}

As discussed in $\S 1$, galaxies in the local universe exhibit a strong correlation between their structure and their star formation history. This section explores this connection using our local galaxy sample. The upper plots in Figure 17 show the distribution of SFR $/ M_{\star}$ vs. $\log n_{i}$ for the full galaxy population, where both the galaxy $1 / V_{\max }$ weighted density distribution and the conditional density distribution are shown. Broadly speaking, there is a clear trend with $\log n_{i}$, with $\mathrm{SFR} / M_{\star}$ decreasing as one moves toward bulge-dominated systems. However, the quintiles clearly demonstrate that the star formation history of disk-dominated galaxies falls within a narrow range of values, whereas bulge-dominated galaxies show a much wider range of SFR $/ M_{\star}$. This demonstrates (1) a dearth of passive disk-dominated galaxies, (2) a significant population of bulge-dominated galaxies with significant SFR $/ M_{\star}$, and (3) a fairly sharp transition between these two zones at $\log n_{i} \sim$ 0.38 . We also reiterate that the "peak" in the distribution of no-SF galaxies at $\log \mathrm{SFR} / M_{\star} \sim-12$ may simply result from the fact that we are unable to measure values of SFR/ $M_{\star}$ significantly below this value; the true distribution is likely to display an even wider dispersion.

One limitation of this plot is that it necessarily combines galaxies with a wide range of $M_{\star}$, which means that differences between low and high stellar mass galaxies may introduce scatter, thereby masking a tighter relation within a single $M_{\star}$ bin. We have found that using narrower ranges in stellar mass does reduce scatter, although in general the overall trends we have identified remain.

We can effectively remove the stellar mass dependence by measuring a quantity that we call the SFR excess, $\Delta_{\mathrm{SFR}}\left(M_{\star}\right)$. We define this using the equation for the ridge line of the SF sequence,

$$
\log \Delta_{\mathrm{SFR}}=\log \mathrm{SFR}-(0.64) \log M_{\star}+6.4,
$$

which provides a measure of the excess or deficiency of SFR as a function of stellar mass.

In certain respects, this residual is similar to the gas deficiency parameter defined by Haynes \& Giovanelli (1984), although it is 

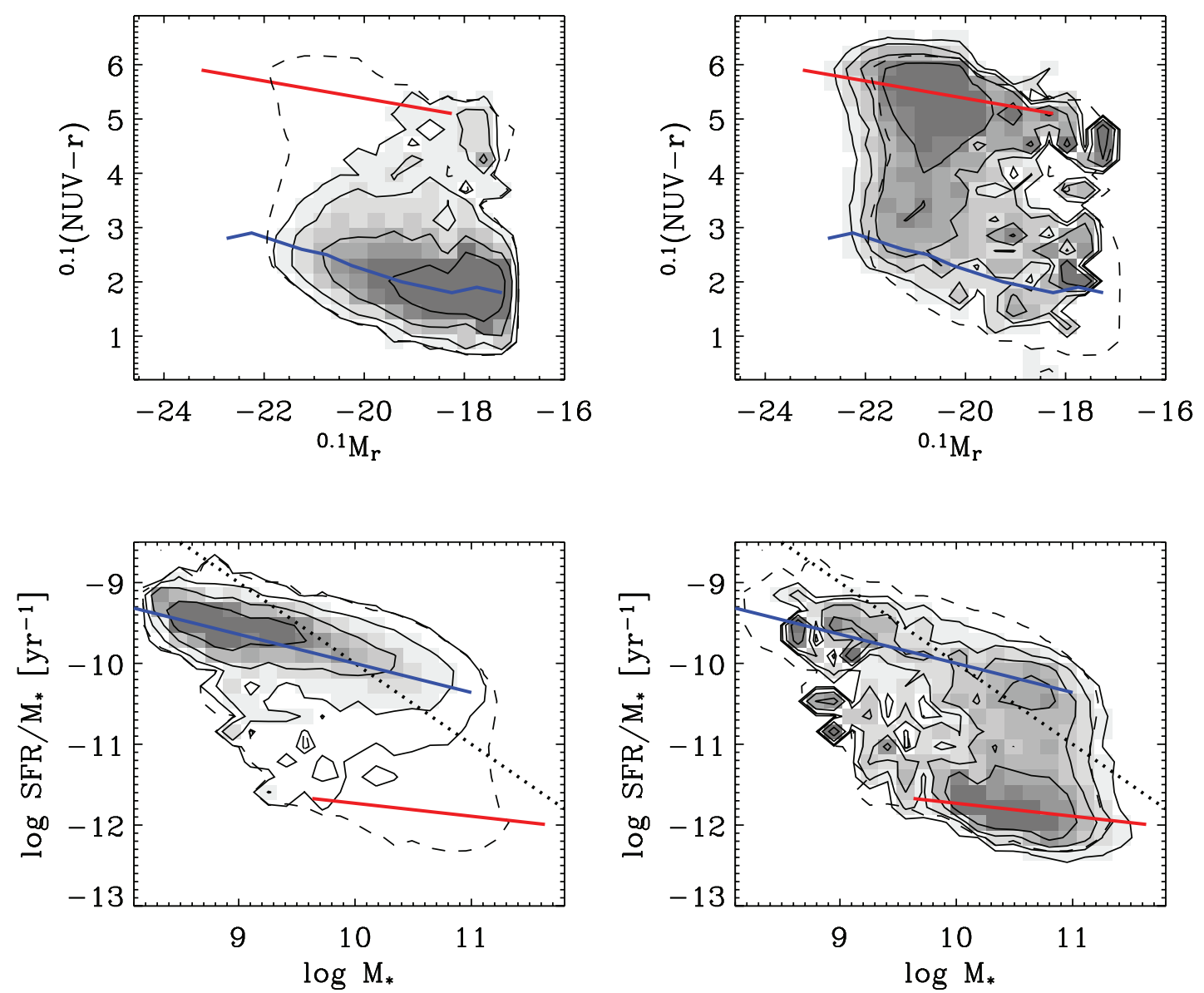

Fig. 18. - Bivariate distribution ( $1 / V_{\max }$-weighted) of galaxies as a function of NUV $-r$ vs. $M_{r}$ (top) and SFR $/ M_{\star}$ vs. $M_{\star}$ (bottom) for subsamples split by Sersic index: $n_{i}<2.4\left(\log n_{i}<0.38\right)($ left $)$ and $n_{i}>2.4\left(\log n_{i}>0.38\right)($ right $)$. Solid lines and contours are as in Fig. 7. Dashed contour follows the outermost contour from Fig. 7.

different in two important ways (other than the trivial sign difference and the fact that we are using SFR rather than gas content to describe an excess). First of all, we correct for a dependence on stellar mass rather than morphological type or the combination of morphological type and radius, as in Solanes et al. (2001). Our method has the advantage that stellar mass is much more reliably measured than morphological type. In addition, we have a basis for physically interpreting the SFR excess, since it can be related (on average) to the SFR intensity, $\Sigma_{\mathrm{SFR}}$, rather than to a median or mean of the full galaxy population in a given stellar mass/luminosity bin.

The SFR excess $\Delta_{\text {SFR }}$ vs. $\log n_{i}$ is plotted in the lower panels of Figure 17. The distribution of $\Delta_{\mathrm{SFR}}$ for disk-dominated galaxies is quite narrow below the disk/bulge transition value. While bulge-dominated galaxies do show a considerable spread in $\Delta_{\mathrm{SFR}}$, there is little variation of $\Delta_{\mathrm{SFR}}$ vs. Sersic index within the separate subpopulations, suggesting an additional controlling parameter for SFR $/ M_{\star}$. See, for example, Blanton et al. (2005a) for an exploration into the connection between Sersic index, color, and environment.

\subsection{SFR $/ M_{\star}$ vs. $M_{\star}$ and $\log \mu_{\star}$ for Disk and Bulge-dominated Subsamples and Derived Gas Fractions}

In this section we use the Sersic index $n_{i}$ to split our main sample into disk and bulge-dominated subsamples in order to investigate the relation between structural properties and the star formation history (and other physical properties) of galaxies in the local universe. Our disk/bulge-dominated cut is similar to the one made in Vincent \& Ryden (2005) and Blanton et al. (2003c). In Figure 18 we present the same plots shown in Figure 7 for each of our subsamples. Disks remain on the blue (and SF) sequence, with a weak tail toward decreasing star formation activity. Most of this tail is at lower stellar masses. We also find that the relative width of the distribution of disk galaxies is wider in uncorrected color than in the SFR $/ M_{\star}$ vs. $M_{\star}$ plot, indicating that much of the scatter in color spread was removed when the dust-attenuation correction was applied. Inclined disks are largely responsible for this spread. Bulge-dominated galaxies, on the other hand, while predominantly populating the no-SF region, show a much greater diversity in color-magnitude and SFR $/ M_{\star}$ vs. $M_{\star}$ space. Some bulge-dominated galaxies appear to have specific star formation rates comparable to disk galaxies, and also populate the SF sequence.

Numerous studies have detected star formation in bulgedominated galaxies, including several key studies in the local universe in the UV (Rich et al. 2005; Yi et al. 2005). Nevertheless, we reiterate the essential qualitative difference between the plots in the lower half of Figure 18 and those from other studies based on the color-magnitude diagram. While disks have been known to occupy the diffuse "blue cloud" of the optical color magnitude diagram (Bell et al. 2005), it is clear that the majority of disks fall on the very tight SF sequence. In fact, it is the bulges, which dominate the red sequence in color-magnitude diagrams, that show a great diversity of SFR $/ M_{\star}$. The majority of bulge-dominated galaxies are not undergoing major episodes of stellar mass buildup. 
Nevertheless, the spread suggests that bulge-dominated galaxies show a rich distribution in SFR despite homogeneity in optical colors and, to some extent, structure.

To quantify this picture, we extracted the one-dimensional $\left(1 / V_{\max }\right)$ distribution of SFR $/ M_{\star}$ in 12 different stellar mass bins, shown in Figure 19. In each plot we show the total distribution, as well as the distribution for disk and bulge-dominated subsamples. For all the samples and subsamples, the $1 \sigma$ confidence interval on the distribution was calculated using bootstrap resampling. We indicate on the top horizontal axis the SFR that corresponds to the equivalent range of SFR $/ M_{\star}$ for that particular $M_{\star}$ bin. A vertical dotted line indicates the location of the SF sequence defined by the relation derived in S07. As expected, these lines intercept the mode of the star-forming peak (SF sequence ridge line) in our derived distributions.

These plots show the relative contribution of disk and bulgedominated galaxies to any part of the SFR $/ M_{\star}$ vs. $M_{\star}$ diagram and further confirm the trends identified above. At low stellar masses the galaxies that lie on the SF sequence are disk-dominated. At higher stellar masses, residual and no-SF galaxies are bulge-dominated. The transition between the prevalence of star-forming and "dead" galaxies appears to take place at a stellar mass of $\log M_{\star}=10.4$. At nearly all stellar masses, bulge-dominated galaxies show a spread in SFR $/ M_{\star}$. Disk galaxies are predominantly located within the SF sequence, although there is evidence for a relative increase in passive disks at low stellar masses. While the relationship between SFR $/ M_{\star}$ and structure is not uniform across all stellar masses, for the $10<\log M_{\star}<11$ range we do find that disk galaxies dominate the SF sequence and bulge-dominated galaxies dominate the residual-SF and no-SF populations. In this stellar mass range, given SFR $/ M_{\star}$, we can predict with high likelihood whether the galaxy is disk or bulgedominated. Figure 19 also shows that at stellar masses above $\log M_{\star}=10.4$, a significant fraction of galaxies in the SF sequence are bulge-dominated galaxies, with these systems becoming the majority at stellar masses above $\log M_{\star}=11.0$. These galaxies are among those with the highest SFRs in the local universe. Extreme versions of these would be the massive, compact UVLGs and/or rare ULIRGs.

Previous work indicates that SFR $/ M_{\star}$ might be better correlated with $\log \mu_{\star}$ than with $M_{\star}$ (Bell \& de Jong 2000; Kauffmann et al. 2003b; Brinchmann et al. 2004). In Figure 20 we show the distribution of SFR $/ M_{\star}$ vs. $\log \mu_{\star}$. On this plot isopleths in $\Sigma_{\mathrm{SFR}}$ lie along straight lines of negative unit slope (under the simplistic assumption that $\left.r_{50, \mathrm{SFR}}=r_{50, r}\right)$. The trends seen in Figure 20 closely match the results from Brinchmann et al. (2004). The most striking feature of these plots is the fact that over a significant range in $\log \mu_{\star}, \mathrm{SFR} / M_{\star}$ is slowly varying (although nearly constant in $\left.\Sigma_{\text {SFR }}\right)$ until the transition point $\log \mu_{\star} \sim 8.5$ beyond which SFR $/ M_{\star}$ varies by several orders of magnitude and $\log \mu_{\star}$ nearly constant. A simple interpretation of this result is that stellar mass surface density increases for star forming galaxies up to a threshold $\log \mu_{\star}$, above which it remains nearly constant. It is intriguing that the highest SFR intensities are observed for galaxies in the range of $\log \mu_{\star}$ where this transition is observed.

We can use our derived physical properties (SFR/ $M_{\star}$ and $\log \mu_{\star}$ ) to attempt to connect our results to the gas content of galaxies. Recently, a number of investigations (Boselli et al. 2001; Bell 2003a; Kannappan 2004; Reddy et al. 2006; Erb et al. 2006) have made use of the correlation between specific star formation rate and/or specific UV luminosities and gas mass fractions. Reddy et al. (2006) obtain a simple expression SFR $/ M_{\star}=C f_{\text {gas }} /\left(1-f_{\text {gas }}\right)$, where the constant $C$ depends on both the constant in the Schmidt law, and the quantity $M_{\text {gas,initial }}=M_{\text {gas }}+M_{\star}$. We simply make use of the global Schmidt law (Kennicutt 1998a) to derive

$$
\Sigma_{\mathrm{SFR}} \propto \Sigma_{\mathrm{gas}}^{\alpha} \propto\left(\frac{M_{\mathrm{gas}, \mathrm{SFR}}}{\pi r_{\mathrm{SFR}}^{2}}\right)^{\alpha} \propto\left(\frac{f_{\mathrm{gas}} \mu_{\star}}{1-f_{\mathrm{gas}}}\right)^{\alpha},
$$

where the gas fraction ${ }^{16} f_{\text {gas }}=M_{\text {gas }} /\left(M_{\star}+M_{\text {gas }}\right)$, and where we have assumed that $r_{\mathrm{SFR}} \sim r_{\star}$ and that all of the gas in the galaxy is involved in recent star formation. For an assumed star formation law (e.g., $\alpha=1.4$ ), we can use $\Sigma_{\text {SFR }}$ and $\log \mu_{\star}$ to estimate the gas fraction in a galaxy. Adopting the star formation law from Kennicutt (1998b), we have

$$
\begin{aligned}
\log \Sigma_{\mathrm{SFR}}= & \log \left(\frac{2.5 \times 10^{-4}}{1.5}\right)+1.4\left(\log \mu_{\star}-6\right) \\
& +1.4 \log \left(\frac{f_{\text {gas }}}{1-f_{\text {gas }}}\right)
\end{aligned}
$$

and

$$
\begin{aligned}
& \log \left(\mathrm{SFR} / M_{\star}\right)=\log \Sigma_{\mathrm{SFR}}-\log \mu \\
& =\log \left(\frac{2.5 \times 10^{-4}}{1.5}\right)+0.4\left(\log \mu_{\star}-6\right)+1.4 \log \left(\frac{f_{\text {gas }}}{1-f_{\text {gas }}}\right) .
\end{aligned}
$$

Figure 20 shows where our estimated gas mass fraction isopleths fall. The transition point which delineates star-forming from nonstar-forming galaxies occurs at $f_{\text {gas }} \sim 0.1$. Most star-forming galaxies have estimated gas mass fractions in the range $0.1<f_{\text {gas }}<$ 0.5 . Residual-SF and non-SF galaxies have $f_{\text {gas }}<0.1$. These estimates suggest that a diminishing gas mass fraction is strongly correlated with a decrease in SF activity in galaxies. We reiterate the caveats that (1) $f_{\text {gas }}$ only includes gas that is traced by star formation, (2) a simple empirical relationship has been used to connect star formation to gas content, and (3) we have employed the optical half-light radius to estimate the half-light radius of the star-forming disk. The first caveat is likely to lead to an underestimate of total gas content of the galaxy, although the amount of gas involved in recent star formation (vs. the gas also being accreted) is itself a physically useful quantity. The effect of the latter two caveats could cause our estimate to be off in either direction. Clearly, these new estimates would benefit from empirical constraints based on resolved and unresolved measurements of cold gas in galaxies. Work along these lines is in progress.

We show in Figure 21 a similar plot, where we have split the population into disks and bulge-dominated galaxies. This split emphasizes the transition described above; disk galaxies show very little variation in SFR $/ M_{\star}$ over a wide range in $\log \mu_{\star}$, and bulgedominated galaxies show little variation in $\log \mu_{\star}$ over a wide range of SFR $/ M_{\star}$. We see that most disk galaxies fall within a narrow range of $\Sigma_{\mathrm{SFR}}$. However, both disks and bulge-dominated galaxies have the highest $\Sigma_{\text {SFR }}$ near the transition $\log \mu_{\star}$. These results are quantitatively illustrated in Figure 22, which divides the complete sample into bins of $\log \mu_{\star}$ in a similar manner as Figure 19. On each plot, the horizontal axis corresponds to SFR $/ M_{\star}$, but at fixed $\log \mu_{\star}$ also corresponds to an axis of $\Sigma_{\mathrm{SFR}}$. We indicate on each plot the average value for the star-forming sequence $\left(\log \Sigma_{\mathrm{SFR}} \sim-1.7\right.$ ), which helps to identify those galaxies that are forming stars at an intensity higher than typical for their measured $M_{\star}$.

\footnotetext{
16 We have adopted the notation $f_{\text {gas }}$ rather than $\mu$ to prevent confusion with $\mu_{\star}$. Some authors define gas fraction as $M_{\mathrm{H}_{\mathrm{I}}} / M_{\star}$.
} 

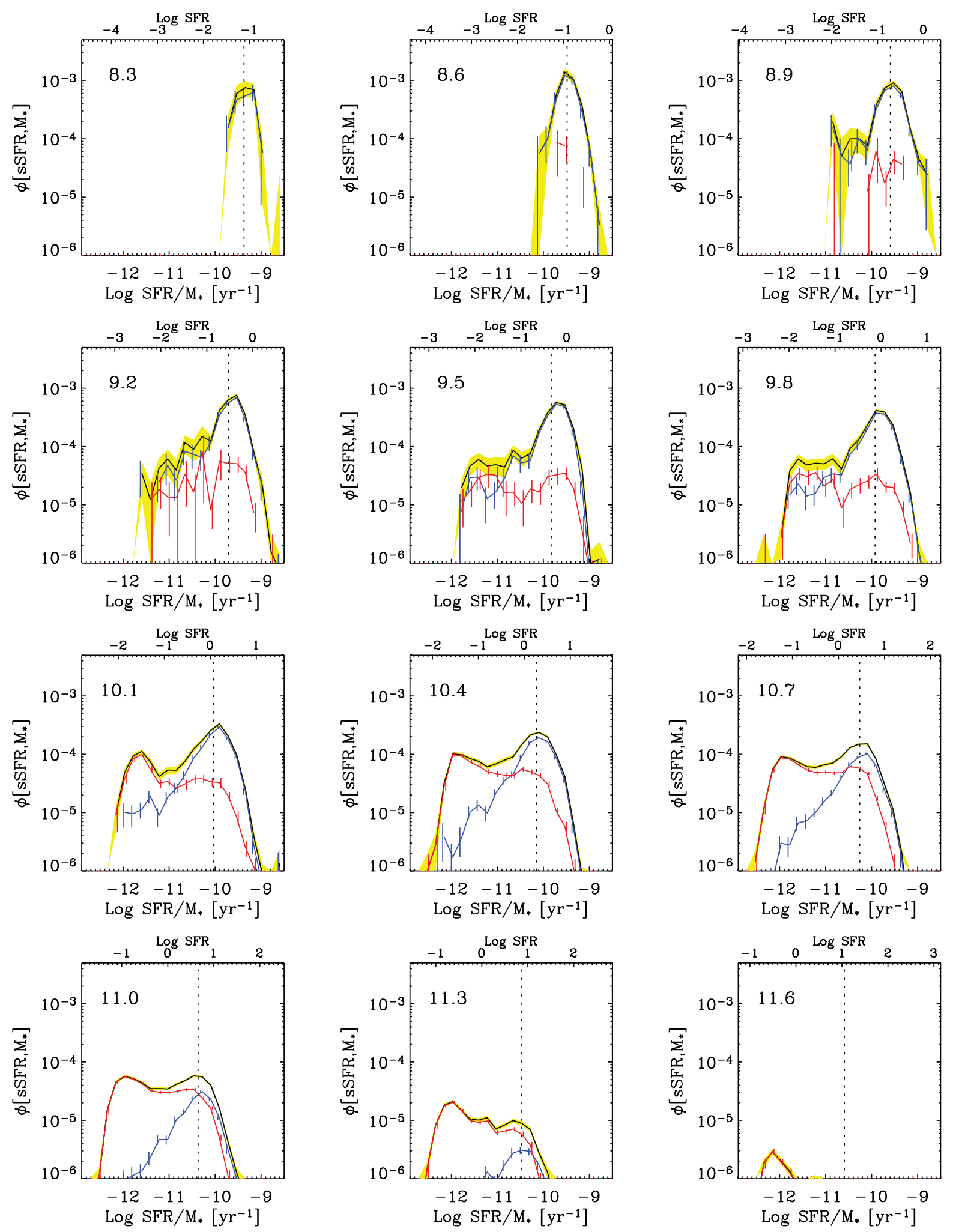

FIG. 19.-Galaxy density distribution $\left(1 / V_{\max }\right.$-weighted) vs. SFR $/ M_{\star}$ in bins of $\log M_{\star}$ for total galaxy sample and in disk/bulge-dominated subsamples split by Sersic index $n_{i}$ (blue/red; split at $n_{i}=2.4$ or $\log n_{i}=0.38$ ). Error bars ( yellow filled regions and vertical lines) have been determined using bootstrap resampling. Upper axis denotes $\log$ SFR using average $\left\langle\log M_{\star}\right\rangle$ (upper left corner of each frame), with the vertical dotted line showing the SFR of the SF sequence ridge for corresponding $\left\langle\log M_{\star}\right\rangle$. Units of $\phi\left(\mathrm{SFR} / M_{\star}, M_{\star}\right)$ are in $\mathrm{Mpc}^{-3} \mathrm{bin}^{-1}$, where each bin is 0.3 dex wide in $M_{\star}$ and 0.1875 dex wide in $\mathrm{SFR} / M_{\star}$. 

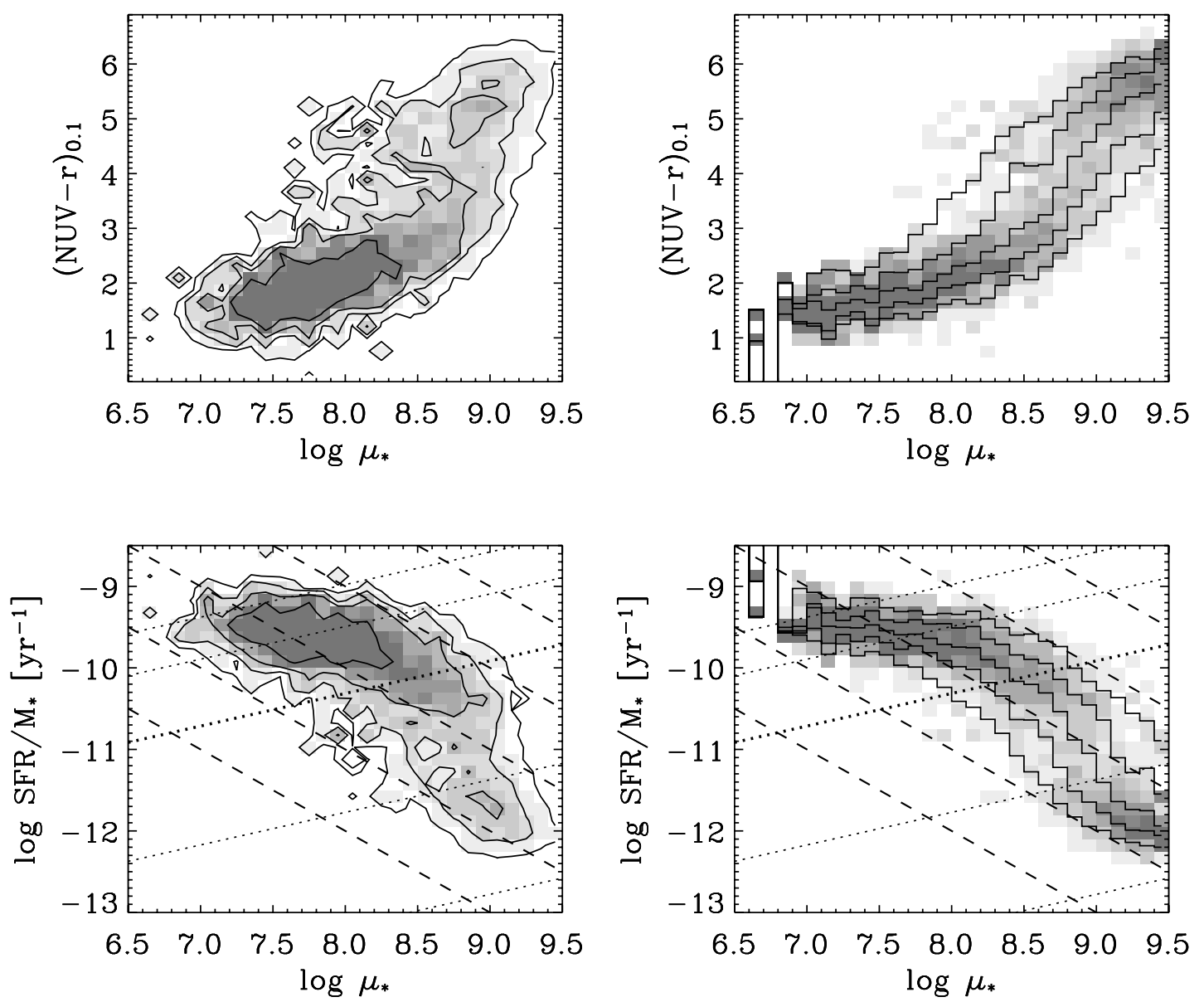

FIG. 20.-UV-optical color and specific star formation rate vs. stellar mass surface density, showing the bivariate distribution $\left(1 / V_{\max }\right.$-weighted) of galaxies as a function of rest-frame NUV $-r$ color (top) and SFR/ $M_{\star}$ (bottom) vs. $\log \mu_{\star}$. For NUV $-r$ vs. $\log \mu_{\star}$, no dust-attenuation correction has been applied to the color. Left: Contours enclose $38 \%, 68 \%, 87 \%$, and $95 \%$ of the distribution. Diagonal dashed lines are isopleths in $\Sigma_{\text {SFR }}$ spaced by 1 dex. Dotted lines show constant gas fractions of (decreasing) $0.5,0.3,0.1,0.01,0.001$. The bold dotted line plots $f_{\text {gas }}=0.1$. Right: Conditional distribution in each $\log \mu_{\star}$ bin. Quintiles represent $10 \%, 25 \%, 50 \%, 75 \%$, and $90 \%$ of the distribution in that bin.
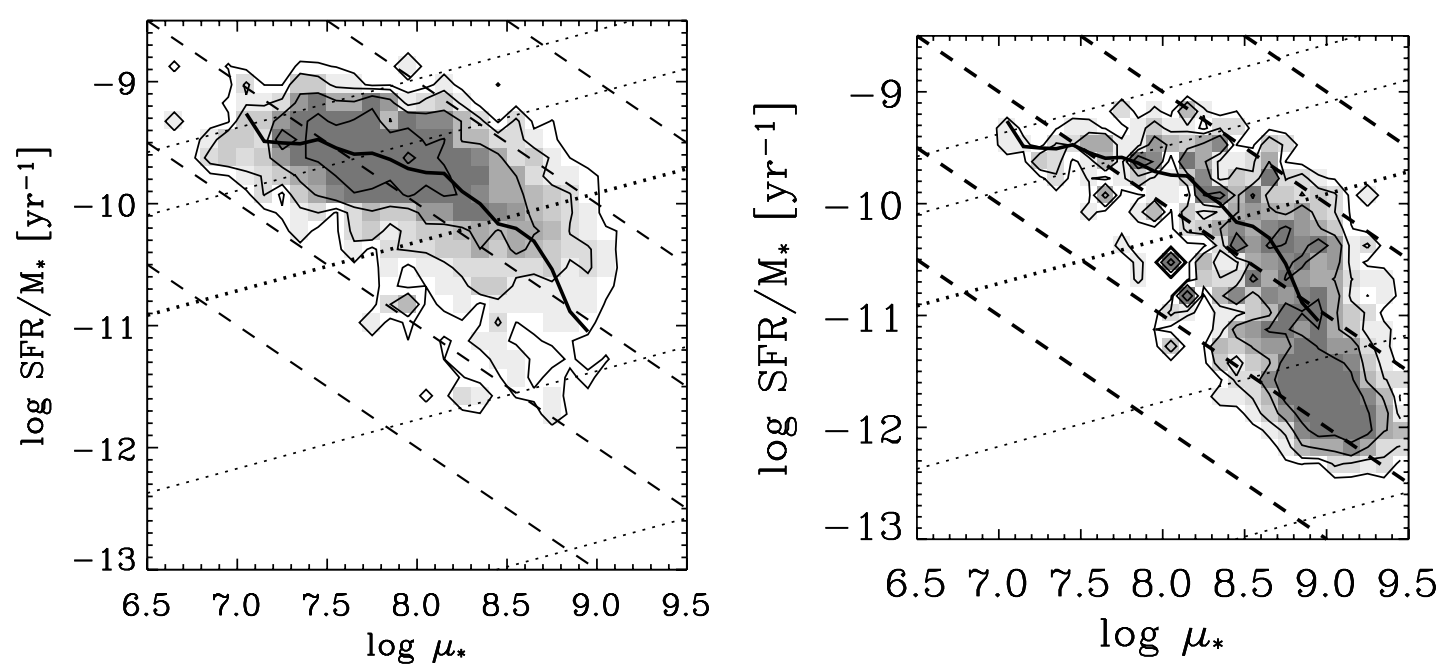

FIG. 21.-UV-optical color and specific star formation rate vs. stellar mass surface density for disks and bulge-dominated galaxies, showing the bivariate distribution $\left(1 / V_{\max }\right.$-weighted) of galaxies as a function of SFR $/ M_{\star}$ vs. $\log \mu_{\star}$ and split by Sersic index $n_{i}: n_{i}<2.4\left(\log n_{i}<0.38\right)(l e f t)$ and $n_{i}>2.4\left(\log n_{i}>0.38\right)($ right $)$. Contours enclose $38 \%, 68 \%, 87 \%$, and $95 \%$ of the distribution. Diagonal dashed lines are isopleths in $\Sigma_{\text {SFR }}$ spaced by 1 dex, dotted lines show constant gas fractions of (decreasing) $0.5,0.3,0.1,0.01,0.001$, bold dotted line plots $f_{\text {gas }}=0.1$, and the solid line shows the median SFR $/ M_{\star}$ vs. $\log \mu_{\star}$ for the full sample. 

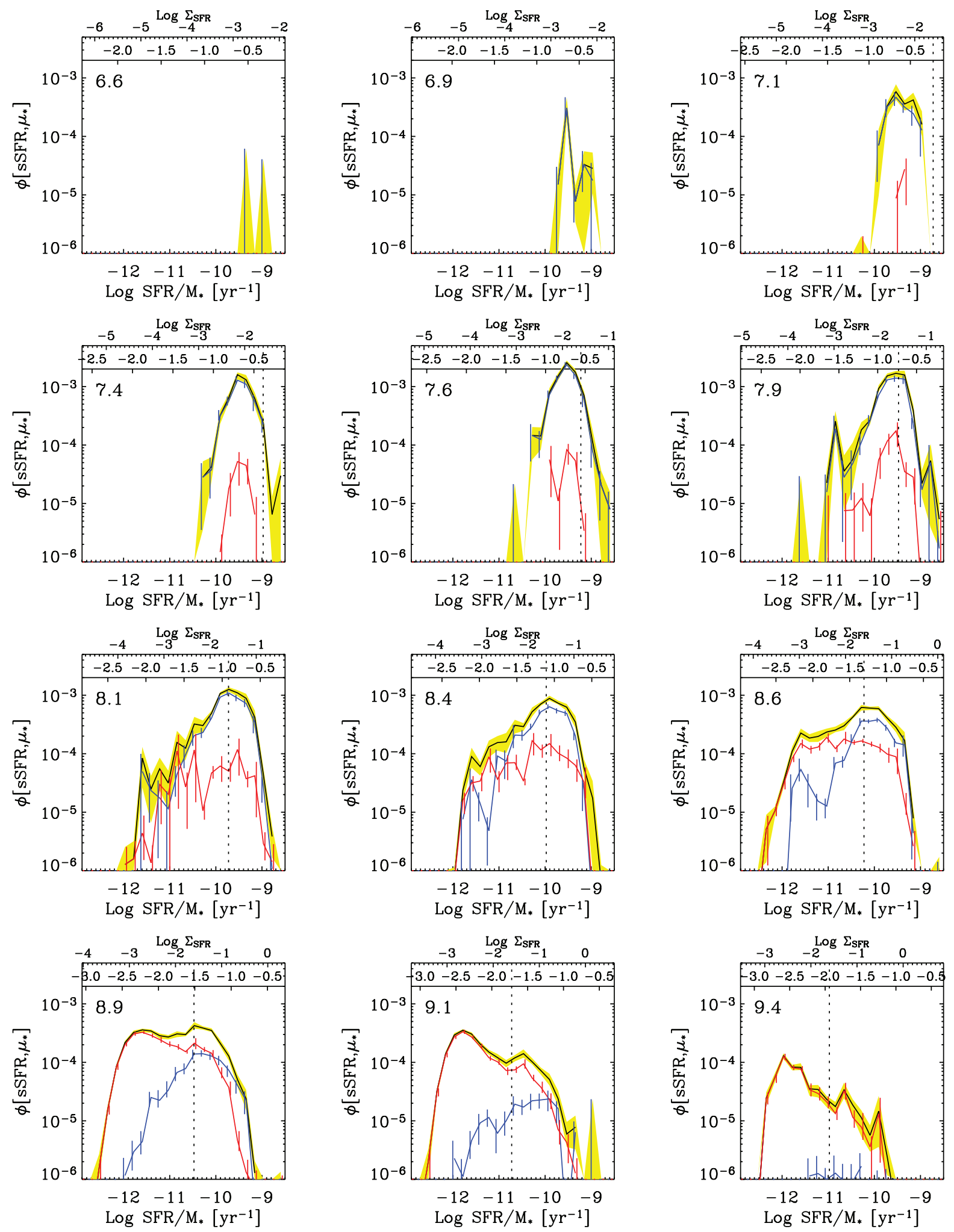

FIG. 22. - SFR $/ M_{\star}$ distribution (1/ $V_{\max }$-weighted) in bins of fixed $\log \mu_{\star}$ for total galaxy sample and in disk/bulge-dominated subsamples split by Sersic index $n_{i}$ (blue/red; split at $n_{i}=2.4$ or $\log n_{i}=0.38$ ). Error bars ( yellow filled regions and vertical lines) have been determined using bootstrap resampling. Upper axes denote star formation rate surface density and gas fraction $\left(\log \Sigma_{\mathrm{SFR}}\right.$ and $\log f_{\mathrm{gas}}$ ) using average $\left\langle\log \mu_{\star}\right\rangle$ (upper left corner of each box), with the vertical dotted line corresponding to $\log \Sigma_{\mathrm{SFR}}=-1.7$. Units of $\phi\left(\mathrm{SFR} / M_{\star}, M_{\star}\right)$ are in $\mathrm{Mpc}^{-3} \operatorname{bin}^{-1}$ where each bin is 0.25 dex wide in $\log \mu_{\star}$ and 0.1875 dex wide in SFR $/ M_{\star}$. 

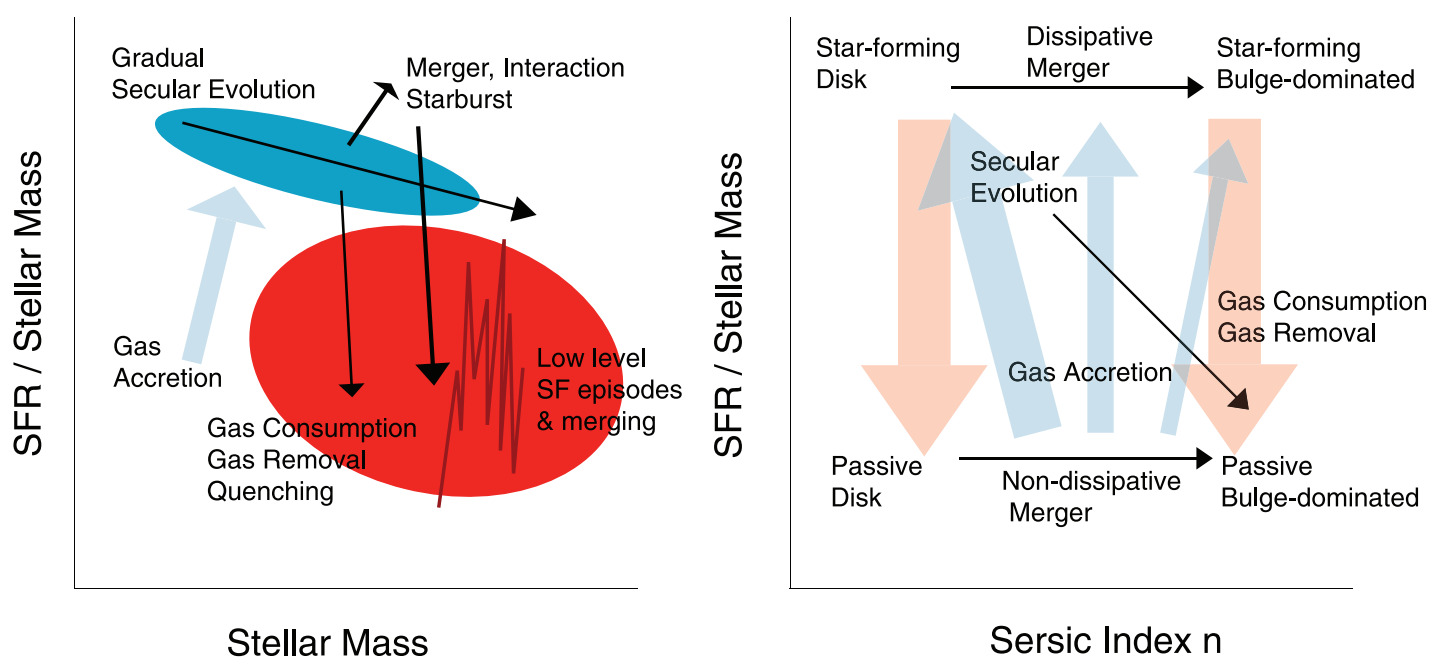

Fig. 23. - Schematic views of star formation history vs. stellar mass (left) and morphology (right, as measured by Sersic index $n$ ) and its evolution. See text for details.

\subsection{On the Evolution of Star-forming Bulge-dominated Galaxies Off of the SF Sequence}

Are star-forming bulge-dominated galaxies plausible candidates for galaxies that are soon to leave the environs of the starforming sequence? In a study of normal and peculiar galaxies (drawn from the Arp atlas), Larson \& Tinsley (1978) found that peculiar galaxies show an increased scatter in their color when compared with normal galaxies, providing evidence for recent bursts. Similarly, we would expect mergers and interactions to trigger star formation activity, resulting in elevated SFRs and/or $\Sigma_{\text {SFR }}$. Could heavily star-forming bulge-dominated galaxies be a short-lived phase in the evolution of a galaxy off of the SF sequence?

Figure 23 (left) provides a schematic view of the SF sequence and possible evolutionary scenarios. Steady star formation can carry a galaxy along the SF sequence, while merging and quenching may push a galaxy off of the SF sequence. The SF sequence itself and physical properties along the sequence are also expected to evolve with time. While gaseous accretion, in some cases via merging, might move a galaxy stochastically through the residual-SF zone and occasionally back onto the SF sequence itself, a plausible track for major and minor mergers is one which takes a galaxy above and below the SF sequence. These and other scenarios may lead to morphological transformations which might be reflected in the relationship between star formation and structure (Fig. 23, right).

The galaxy distribution function presented in Figure 19 and Table 3, combined with very simple assumptions regarding the evolution of star-forming bulge-dominated galaxies, can be used to quantify the rate at which galaxies might be leaving the SF sequence. We can compare our estimates with other measurements to test whether or not these bulge-dominated galaxies might constitute a significant fraction of the population being quenched. One strength of this approach is that it uses measurements of the galaxies with the highest SFR (and $L_{\mathrm{bol}}$ ), a fact that could facilitate its application at higher redshift. This approach does rely on accurately characterizing the distributions of galaxies along the SF sequence (as has been done by Noeske et al. 2007a; Labbe et al. 2007).

Figure 24 shows the integrated number of galaxies,

$\Phi\left(>\mathrm{SFR} / M_{\star}, M_{\star}\right)=\int_{\mathrm{SFR} / M_{\star}}^{\infty} \phi\left(\mathrm{SFR} / M_{\star}, M_{\star}\right) d\left(\mathrm{SFR} / M_{\star}\right)$, in each stellar mass bin with SFR $/ M_{\star}$ greater than a specified value. In the limit SFR $/ M_{\star} \rightarrow 0, \Phi\left(>\mathrm{SFR} / M_{\star}, M_{\star}\right) \rightarrow \phi\left(M_{\star}\right)$, which shows good agreement with the local stellar mass function derived by Borch et al. (2006) (taken from Bell et al. 2003b). This function will be used to determine the total volume density of (e.g., bulge-dominated) galaxies of a particular $M_{\star}$ forming stars at a rate higher than galaxies on the SF sequence.

We then make three simple assumptions:

1. The end state of a galaxy leaving the SF sequence ("quenched galaxy") is a bulge-dominated galaxy. As discussed above, passive disks are rare.

2. All galaxies leaving the $S F$ sequence experience a brief period of elevated SFR for their given $M_{\star}$. Models and observations suggest that most processes that lead to morphological transformation and quenching (interactions, mergers with possible feedback) experience periods of significantly enhanced star formation. Our method will not identify galaxies that do not experience this phase.

3. All bulge-dominated galaxies with elevated SFR will be quenched. For our simple analysis, we assume elevated SFR occurs at $\log \Delta_{\text {SFR }}>0.5$ and that $\tau_{\text {burst }}=0.5$ Gyr. We discuss the implications of this third assumption below.

As a check on our method, we can compare the number rate density for the production of star-forming bulge-dominated galaxies to the merger rate by assuming that bursts result from mergers and that we have relative steady state over short timescales (e.g., $\dot{n}_{\mathrm{SF}, \downarrow} \simeq \dot{n}_{\text {merger }}$ ). We calculate the number rate density,

$$
\begin{aligned}
& \dot{n}_{\mathrm{SF}, \downarrow}\left(>M_{\star}\right)= \\
& \frac{\int_{M_{\star}}^{\infty} \int_{\mathrm{SFR} / M_{\star}}^{\infty} \phi\left(\mathrm{SFR} / M_{\star}, M_{\star}\right) d\left(\mathrm{SFR} / M_{\star}\right) d\left(M_{\star}\right)}{\tau_{\text {burst }}},
\end{aligned}
$$

and the bursting fraction,

$$
\begin{aligned}
& \operatorname{Frac}_{\mathrm{SF}, \downarrow}\left(>M_{\star}\right)= \\
& \frac{\int_{M_{\star}}^{\infty} \int_{\mathrm{SFR} / M_{\star}}^{\infty} \phi\left(\mathrm{SFR} / M_{\star}, M_{\star}\right) d\left(\mathrm{SFR} / M_{\star}\right) d\left(M_{\star}\right)}{\int_{M_{\star}}^{\infty} \phi\left(M_{\star}\right) d\left(M_{\star}\right)},
\end{aligned}
$$

which are shown in Figure 25 (in plotting both quantities we have multiplied by a factor of 2 in order to compare to merger rates and fractions in the literature, which are based on premerger 
TABLE 3

$\phi\left(\mathrm{SFR} / M_{\star}, M_{\star}\right)$ For Total, Disk and Bulge-Dominated Subsamples

\begin{tabular}{|c|c|c|c|c|c|c|}
\hline $\log M_{\star}$ & $\log \mathrm{SFR} / M_{\star}$ & Total & $\begin{array}{c}\log \phi\left(\mathrm{SFR} / M_{\star}, M_{\star}\right)^{\mathrm{a}} \\
n_{i}<2.4\end{array}$ & $n_{i}>2.4$ & $\log \Delta$ SFR & $\begin{array}{l}\text { Fraction } \\
n_{i}>2.4\end{array}$ \\
\hline $8.25 \ldots \ldots \ldots \ldots$. & -9.72 & $-3.82 \pm 0.21$ & $-3.82 \pm 0.21$ & $\ldots$ & -0.35 & $\ldots$ \\
\hline $8.25 \ldots \ldots \ldots-1$ & -9.53 & $-3.22 \pm 0.13$ & $-3.33 \pm 0.15$ & $\ldots$ & -0.16 & $\ldots$ \\
\hline $8.25 \ldots \ldots \ldots \ldots \ldots \ldots \ldots \ldots \ldots$ & -9.34 & $-3.12 \pm 0.11$ & $-3.26 \pm 0.12$ & $\ldots$ & 0.03 & $\ldots$ \\
\hline 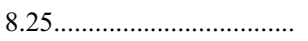 & -9.16 & $-3.16 \pm 0.11$ & $-3.20 \pm 0.12$ & $\ldots$ & 0.22 & $\ldots$ \\
\hline 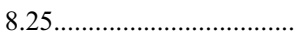 & -8.97 & $-4.25 \pm 0.27$ & $-4.25 \pm 0.27$ & $\ldots$ & 0.40 & $\ldots$ \\
\hline 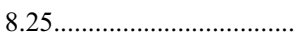 & -8.59 & $-4.90 \pm 0.30$ & $\ldots$ & $\ldots$ & 0.78 & $\ldots$ \\
\hline 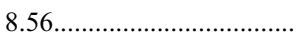 & -10.09 & $-4.26 \pm 0.30$ & $-4.26 \pm 0.30$ & $\ldots$ & -0.61 & $\ldots$ \\
\hline 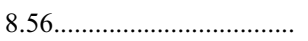 & -9.91 & $-4.00 \pm 0.22$ & $-4.00 \pm 0.21$ & $\ldots$ & -0.42 & $\ldots$ \\
\hline 8.56 & -9.72 & $-3.25 \pm 0.10$ & $-3.32 \pm 0.11$ & $-4.06 \pm 0.22$ & -0.24 & 0.15 \\
\hline 8.56 & -9.53 & $-2.86 \pm 0.06$ & $-2.91 \pm 0.06$ & $-4.12 \pm 0.17$ & -0.05 & 0.06 \\
\hline 8.56 & -9.34 & $-2.98 \pm 0.07$ & $-2.99 \pm 0.07$ & $\ldots$ & 0.14 & $\ldots$ \\
\hline 8.56 & -9.16 & $-3.44 \pm 0.08$ & $-3.55 \pm 0.08$ & $-4.71 \pm 0.22$ & 0.33 & 0.05 \\
\hline 8.56 & -8.97 & $-4.26 \pm 0.15$ & $-4.26 \pm 0.15$ & $\ldots$ & 0.51 & $\ldots$ \\
\hline $8.56 \ldots \ldots \ldots \ldots-1$. & -8.78 & $-5.47 \pm 0.30$ & $-5.47 \pm 0.30$ & $\ldots$ & 0.70 & \\
\hline $8.87 \ldots \ldots \ldots \ldots \ldots \ldots \ldots . .$. & -10.84 & $-3.70 \pm 0.17$ & $-3.81 \pm 0.18$ & $-4.38 \pm 0.30$ & -1.25 & 0.21 \\
\hline 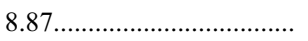 & -10.66 & $-4.29 \pm 0.30$ & $-4.29 \pm 0.30$ & $\ldots$ & -1.06 & $\ldots$ \\
\hline 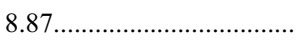 & -10.47 & $-4.00 \pm 0.17$ & $-4.43 \pm 0.23$ & $-4.20 \pm 0.21$ & -0.88 & 0.63 \\
\hline 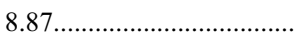 & -10.28 & $-4.00 \pm 0.16$ & $-4.00 \pm 0.16$ & $\ldots$ & -0.69 & $\ldots$ \\
\hline 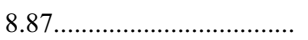 & -10.09 & $-4.12 \pm 0.15$ & $-4.19 \pm 0.16$ & $-4.90 \pm 0.30$ & -0.50 & 0.17 \\
\hline 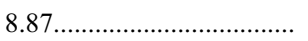 & -9.91 & $-3.43 \pm 0.09$ & $-3.51 \pm 0.09$ & $-4.22 \pm 0.23$ & -0.31 & 0.16 \\
\hline 8.87 & -9.72 & $-3.13 \pm 0.05$ & $-3.17 \pm 0.05$ & $-4.76 \pm 0.20$ & -0.13 & 0.02 \\
\hline 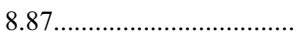 & -9.53 & $-3.04 \pm 0.04$ & $-3.10 \pm 0.04$ & $-4.35 \pm 0.15$ & 0.06 & 0.05 \\
\hline 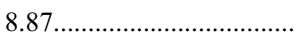 & -9.34 & $-3.19 \pm 0.04$ & $-3.26 \pm 0.05$ & $-4.44 \pm 0.16$ & 0.25 & 0.06 \\
\hline 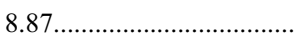 & -9.16 & $-3.85 \pm 0.07$ & $-3.87 \pm 0.07$ & $\ldots$ & 0.44 & $\ldots$ \\
\hline 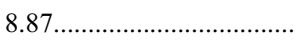 & -8.97 & $-4.35 \pm 0.12$ & $-4.44 \pm 0.13$ & $\ldots$ & 0.62 & $\ldots$ \\
\hline 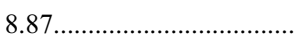 & -8.78 & $-4.62 \pm 0.28$ & $-4.62 \pm 0.27$ & $\ldots$ & 0.81 & $\ldots$ \\
\hline $9.18 \ldots \ldots \ldots \ldots \ldots \ldots . .$. & -11.59 & $-4.46 \pm 0.21$ & $-4.46 \pm 0.21$ & $\ldots$ & -1.89 & $\ldots$ \\
\hline 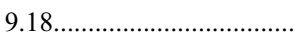 & -11.41 & $-4.92 \pm 0.30$ & $\ldots$ & $-4.92 \pm 0.30$ & -1.70 & 1.00 \\
\hline 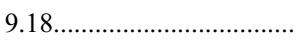 & -11.22 & $-4.37 \pm 0.17$ & $-4.61 \pm 0.20$ & $-4.74 \pm 0.19$ & -1.51 & 0.43 \\
\hline 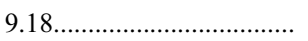 & -11.03 & $-4.21 \pm 0.16$ & $-4.32 \pm 0.19$ & $-4.86 \pm 0.25$ & -1.33 & 0.22 \\
\hline 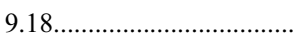 & -10.84 & $-4.40 \pm 0.18$ & $-4.58 \pm 0.20$ & $-4.88 \pm 0.30$ & -1.14 & 0.34 \\
\hline 9.18 & -10.66 & $-3.93 \pm 0.12$ & $-4.08 \pm 0.15$ & $-4.47 \pm 0.19$ & -0.95 & 0.29 \\
\hline 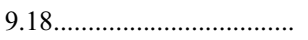 & -10.47 & $-4.05 \pm 0.15$ & $-4.14 \pm 0.16$ & $-4.78 \pm 0.30$ & -0.76 & 0.18 \\
\hline 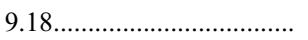 & -10.28 & $-3.83 \pm 0.18$ & $-4.19 \pm 0.14$ & $-4.08 \pm 0.27$ & -0.58 & 0.56 \\
\hline 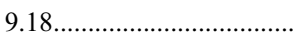 & -10.09 & $-3.91 \pm 0.10$ & $-3.94 \pm 0.11$ & $-5.10 \pm 0.18$ & -0.39 & 0.07 \\
\hline 9.18 & -9.91 & $-3.38 \pm 0.05$ & $-3.47 \pm 0.06$ & $-4.25 \pm 0.19$ & -0.20 & 0.13 \\
\hline 9.18 & -9.72 & $-3.21 \pm 0.04$ & $-3.25 \pm 0.04$ & $-4.29 \pm 0.12$ & -0.01 & 0.08 \\
\hline $9.18 \ldots \ldots \ldots \ldots \ldots \ldots . .$. & -9.53 & $-3.12 \pm 0.03$ & $-3.16 \pm 0.03$ & $-4.29 \pm 0.11$ & 0.17 & 0.07 \\
\hline 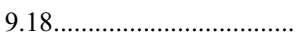 & -9.34 & $-3.46 \pm 0.04$ & $-3.53 \pm 0.04$ & $-4.44 \pm 0.12$ & 0.36 & 0.10 \\
\hline 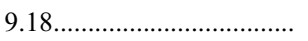 & -9.16 & $-4.10 \pm 0.07$ & $-4.15 \pm 0.07$ & $-5.15 \pm 0.18$ & 0.55 & 0.09 \\
\hline 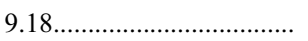 & -8.97 & $-4.69 \pm 0.15$ & $-4.69 \pm 0.15$ & & 0.74 & \\
\hline 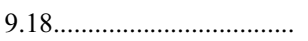 & -8.78 & $-5.80 \pm 0.30$ & $\ldots$ & $-5.80 \pm 0.30$ & 0.92 & 1.00 \\
\hline 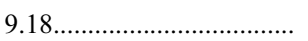 & -8.59 & $-6.14 \pm 0.30$ & $-6.14 \pm 0.30$ & $\ldots$ & 1.11 & $\ldots$ \\
\hline 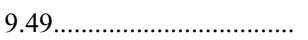 & -11.78 & $-4.71 \pm 0.20$ & $-5.11 \pm 0.30$ & $-4.93 \pm 0.22$ & -1.97 & 0.60 \\
\hline 9.49 & -11.59 & $-4.33 \pm 0.13$ & $-4.53 \pm 0.16$ & $-4.76 \pm 0.17$ & -1.78 & 0.37 \\
\hline 9.49 & -11.41 & $-4.22 \pm 0.11$ & $-4.52 \pm 0.16$ & $-4.53 \pm 0.13$ & -1.59 & 0.50 \\
\hline 9.49 & -11.22 & $-4.33 \pm 0.13$ & $-4.89 \pm 0.21$ & $-4.47 \pm 0.15$ & -1.40 & 0.72 \\
\hline 9.49 & -11.03 & $-4.31 \pm 0.12$ & $-4.77 \pm 0.18$ & $-4.49 \pm 0.15$ & -1.22 & 0.66 \\
\hline 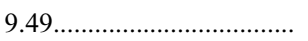 & -10.84 & $-4.35 \pm 0.14$ & $-4.55 \pm 0.17$ & $-4.79 \pm 0.20$ & -1.03 & 0.36 \\
\hline 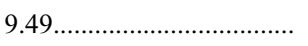 & -10.66 & $-4.07 \pm 0.10$ & $-4.16 \pm 0.11$ & $-4.78 \pm 0.16$ & -0.84 & 0.19 \\
\hline 9.49 & -10.47 & $-4.20 \pm 0.11$ & $-4.28 \pm 0.13$ & $-4.98 \pm 0.22$ & -0.65 & 0.17 \\
\hline 9.49 & -10.28 & $-4.12 \pm 0.09$ & $-4.24 \pm 0.11$ & $-4.73 \pm 0.15$ & -0.47 & 0.25 \\
\hline $9.49 \ldots \ldots \ldots \ldots \ldots \ldots . .$. & -10.09 & $-3.72 \pm 0.06$ & $-3.77 \pm 0.06$ & $-4.78 \pm 0.14$ & -0.28 & 0.09 \\
\hline 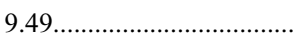 & -9.91 & $-3.42 \pm 0.04$ & $-3.48 \pm 0.04$ & $-4.51 \pm 0.12$ & -0.09 & 0.08 \\
\hline $9.49 \ldots \ldots \ldots-1 \ldots$ & -9.72 & $-3.25 \pm 0.03$ & $-3.28 \pm 0.03$ & $-4.49 \pm 0.13$ & 0.10 & 0.06 \\
\hline 9.49 & -9.53 & $-3.28 \pm 0.03$ & $-3.32 \pm 0.03$ & $-4.46 \pm 0.09$ & 0.28 & 0.07 \\
\hline 9.49 & -9.34 & $-3.69 \pm 0.04$ & $-3.76 \pm 0.04$ & $-4.71 \pm 0.12$ & 0.47 & 0.10 \\
\hline 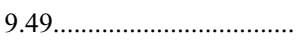 & -9.16 & $-4.39 \pm 0.13$ & $-4.69 \pm 0.11$ & $-5.44 \pm 0.25$ & 0.66 & 0.09 \\
\hline 9.49 & -8.97 & $-6.01 \pm 0.21$ & $-6.36 \pm 0.30$ & $-6.26 \pm 0.30$ & 0.85 & 0.56 \\
\hline 9.49 & -8.78 & $-5.93 \pm 0.18$ & $-6.24 \pm 0.30$ & $-6.22 \pm 0.30$ & 1.03 & 0.51 \\
\hline 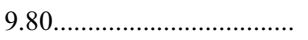 & -12.34 & $-5.80 \pm 0.30$ & $\ldots$ & $-5.80 \pm 0.30$ & -2.42 & 1.00 \\
\hline $9.80 \ldots \ldots \ldots \ldots . .$. & -11.97 & $-5.56 \pm 0.20$ & $\ldots$ & $-5.56 \pm 0.20$ & -2.04 & 1.00 \\
\hline 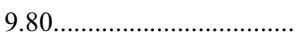 & -11.78 & $-4.42 \pm 0.10$ & $-4.83 \pm 0.16$ & $-4.63 \pm 0.11$ & -1.85 & 0.62 \\
\hline 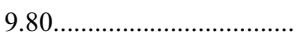 & -11.59 & $-4.22 \pm 0.08$ & $-4.64 \pm 0.13$ & $-4.46 \pm 0.09$ & -1.67 & 0.57 \\
\hline
\end{tabular}


TABLE 3-Continued

\begin{tabular}{|c|c|c|c|c|c|c|}
\hline $\log M_{\star}$ & $\log \mathrm{SFR} / M_{\star}$ & Total & $\begin{array}{c}\log \phi\left(\mathrm{SFR} / M_{\star}, M_{\star}\right)^{\mathrm{a}} \\
n_{i}<2.4\end{array}$ & $n_{i}>2.4$ & $\log \Delta$ SFR & $\begin{array}{l}\text { Fraction } \\
n_{i}>2.4\end{array}$ \\
\hline 9.80 & -11.41 & $-4.32 \pm 0.09$ & $-4.85 \pm 0.16$ & $-4.51 \pm 0.10$ & -1.48 & 0.63 \\
\hline 9.80 & -11.22 & $-4.29 \pm 0.09$ & $-4.80 \pm 0.14$ & $-4.44 \pm 0.10$ & -1.29 & 0.69 \\
\hline 9.80 & -11.03 & $-4.30 \pm 0.08$ & $-4.53 \pm 0.11$ & $-4.68 \pm 0.12$ & -1.10 & 0.41 \\
\hline $9.80 \ldots \ldots \ldots$ & -10.84 & $-4.21 \pm 0.08$ & $-4.47 \pm 0.12$ & $-4.56 \pm 0.12$ & -0.92 & 0.45 \\
\hline $9.80 \ldots \ldots \ldots$ & -10.66 & $-4.38 \pm 0.11$ & $-4.49 \pm 0.12$ & $-5.05 \pm 0.18$ & -0.73 & 0.21 \\
\hline 9.80 & -10.47 & $-4.04 \pm 0.07$ & $-4.13 \pm 0.08$ & $-4.73 \pm 0.12$ & -0.54 & 0.20 \\
\hline 9.80 & -10.28 & $-3.88 \pm 0.07$ & $-3.97 \pm 0.08$ & $-4.66 \pm 0.11$ & -0.35 & 0.17 \\
\hline 9.80 & -10.09 & $-3.63 \pm 0.04$ & $-3.70 \pm 0.04$ & $-4.59 \pm 0.10$ & -0.17 & 0.11 \\
\hline 9.80 & -9.91 & $-3.38 \pm 0.02$ & $-3.43 \pm 0.03$ & $-4.47 \pm 0.10$ & 0.02 & 0.08 \\
\hline 9.80 & -9.72 & $-3.41 \pm 0.02$ & $-3.45 \pm 0.02$ & $-4.69 \pm 0.09$ & 0.21 & 0.05 \\
\hline 9.80 & -9.53 & $-3.64 \pm 0.03$ & $-3.69 \pm 0.03$ & $-4.70 \pm 0.08$ & 0.40 & 0.09 \\
\hline 9.80 & -9.34 & $-4.18 \pm 0.04$ & $-4.25 \pm 0.04$ & $-5.05 \pm 0.11$ & 0.58 & 0.13 \\
\hline 9.80 & -9.16 & $-4.80 \pm 0.07$ & $-4.86 \pm 0.08$ & $-5.66 \pm 0.16$ & 0.77 & 0.14 \\
\hline 9.80 & -8.97 & $-5.83 \pm 0.18$ & $-5.83 \pm 0.18$ & $\ldots$ & 0.96 & $\ldots$ \\
\hline 9.80 & -8.78 & $-6.58 \pm 0.30$ & $-6.58 \pm 0.30$ & $\ldots$ & 1.15 & $\ldots$ \\
\hline $10.10 \ldots \ldots \ldots \ldots$ & -12.16 & $-5.04 \pm 0.17$ & & $-5.04 \pm 0.16$ & -2.12 & 1.00 \\
\hline $10.10 \ldots \ldots \ldots \ldots \ldots$ & -11.97 & $-4.32 \pm 0.06$ & $-5.00 \pm 0.16$ & $-4.42 \pm 0.07$ & -1.93 & 0.79 \\
\hline $10.10 \ldots \ldots \ldots \ldots \ldots \ldots . .$. & -11.78 & $-4.03 \pm 0.05$ & $-5.02 \pm 0.14$ & $-4.08 \pm 0.05$ & -1.74 & 0.90 \\
\hline 10.10 & -11.59 & $-3.95 \pm 0.04$ & $-4.94 \pm 0.12$ & $-4.01 \pm 0.04$ & -1.56 & 0.88 \\
\hline 10.10 & -11.41 & $-4.13 \pm 0.05$ & $-4.72 \pm 0.09$ & $-4.26 \pm 0.06$ & -1.37 & 0.74 \\
\hline $10.10 \ldots \ldots \ldots \ldots$ & -11.22 & $-4.38 \pm 0.06$ & $-5.05 \pm 0.13$ & $-4.49 \pm 0.07$ & -1.18 & 0.77 \\
\hline 10.10 & -11.03 & $-4.27 \pm 0.05$ & $-4.74 \pm 0.10$ & $-4.47 \pm 0.07$ & -0.99 & 0.64 \\
\hline $10.10 \ldots \ldots \ldots \ldots \ldots$ & -10.84 & $-4.28 \pm 0.06$ & $-4.58 \pm 0.09$ & $-4.58 \pm 0.07$ & -0.81 & 0.50 \\
\hline $10.10 \ldots \ldots \ldots \ldots \ldots \ldots \ldots$ & -10.66 & $-4.12 \pm 0.05$ & $-4.35 \pm 0.07$ & $-4.51 \pm 0.07$ & -0.62 & 0.41 \\
\hline $10.10 \ldots \ldots \ldots \ldots \ldots \ldots \ldots$ & -10.47 & $-3.91 \pm 0.04$ & $-4.07 \pm 0.05$ & $-4.42 \pm 0.07$ & -0.43 & 0.31 \\
\hline $10.10 \ldots \ldots \ldots \ldots \ldots$ & -10.28 & $-3.77 \pm 0.03$ & $-3.88 \pm 0.04$ & $-4.42 \pm 0.07$ & -0.24 & 0.22 \\
\hline $10.10 \ldots \ldots \ldots \ldots \ldots \ldots \ldots$ & -10.09 & $-3.59 \pm 0.02$ & $-3.66 \pm 0.03$ & $-4.47 \pm 0.06$ & -0.06 & 0.13 \\
\hline $10.10 \ldots \ldots \ldots \ldots \ldots$ & -9.91 & $-3.48 \pm 0.02$ & $-3.54 \pm 0.02$ & $-4.49 \pm 0.08$ & 0.13 & 0.10 \\
\hline $10.10 \ldots \ldots \ldots \ldots \ldots$ & -9.72 & $-3.68 \pm 0.02$ & $-3.74 \pm 0.02$ & $-4.71 \pm 0.07$ & 0.32 & 0.09 \\
\hline $10.10 \ldots \ldots \ldots \ldots \ldots \ldots \ldots$ & -9.53 & $-4.01 \pm 0.03$ & $-4.06 \pm 0.03$ & $-5.08 \pm 0.09$ & 0.51 & 0.09 \\
\hline 10.10 & -9.34 & $-4.58 \pm 0.05$ & $-4.63 \pm 0.05$ & $-5.59 \pm 0.11$ & 0.69 & 0.10 \\
\hline 10.10 & -9.16 & $-5.41 \pm 0.09$ & $-5.57 \pm 0.10$ & $-5.93 \pm 0.16$ & 0.88 & 0.30 \\
\hline $10.10 \ldots \ldots \ldots$ & -8.97 & $-6.06 \pm 0.18$ & $-6.25 \pm 0.21$ & $-6.52 \pm 0.20$ & 1.07 & 0.35 \\
\hline 10.10 & -8.78 & $-6.86 \pm 0.30$ & $-6.86 \pm 0.30$ & & 1.26 & $\ldots$ \\
\hline 10.10 & -8.59 & $-5.86 \pm 0.23$ & $-5.93 \pm 0.25$ & $-6.68 \pm 0.30$ & 1.44 & 0.15 \\
\hline $10.41 \ldots \ldots \ldots \ldots$ & -12.53 & $-6.02 \pm 0.30$ & $\ldots$ & $-6.02 \pm 0.30$ & -2.38 & 1.00 \\
\hline $10.41 \ldots \ldots \ldots$ & -12.34 & $-5.56 \pm 0.20$ & $\ldots$ & $-5.56 \pm 0.22$ & -2.20 & 1.00 \\
\hline 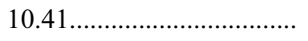 & -12.16 & $-4.49 \pm 0.07$ & $-5.42 \pm 0.21$ & $-4.54 \pm 0.07$ & -2.01 & 0.88 \\
\hline $10.41 \ldots \ldots \ldots \ldots \ldots \ldots$ & -11.97 & $-3.99 \pm 0.03$ & $-5.76 \pm 0.21$ & $-4.00 \pm 0.03$ & -1.82 & 0.98 \\
\hline $10.41 \ldots \ldots \ldots \ldots$ & -11.78 & $-4.01 \pm 0.03$ & $-5.44 \pm 0.17$ & $-4.03 \pm 0.03$ & -1.63 & 0.95 \\
\hline 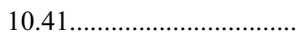 & -11.59 & $-4.07 \pm 0.03$ & $-4.99 \pm 0.09$ & $-4.13 \pm 0.03$ & -1.45 & 0.87 \\
\hline 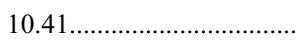 & -11.41 & $-4.12 \pm 0.03$ & $-4.87 \pm 0.08$ & $-4.21 \pm 0.03$ & -1.26 & 0.82 \\
\hline $10.41 \ldots \ldots \ldots$ & -11.22 & $-4.22 \pm 0.04$ & $-5.01 \pm 0.11$ & $-4.30 \pm 0.04$ & -1.07 & 0.83 \\
\hline $10.41 \ldots \ldots \ldots$ & -11.03 & $-4.17 \pm 0.04$ & $-4.66 \pm 0.07$ & $-4.33 \pm 0.05$ & -0.88 & 0.68 \\
\hline $10.41 \ldots \ldots \ldots \ldots \ldots \ldots$ & -10.84 & $-4.10 \pm 0.04$ & $-4.45 \pm 0.06$ & $-4.36 \pm 0.05$ & -0.70 & 0.55 \\
\hline 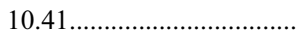 & -10.66 & $-4.05 \pm 0.04$ & $-4.34 \pm 0.05$ & $-4.37 \pm 0.04$ & -0.51 & 0.47 \\
\hline $10.41 \ldots \ldots \ldots \ldots \ldots \ldots . .$. & -10.47 & $-3.83 \pm 0.03$ & $-4.04 \pm 0.03$ & $-4.25 \pm 0.04$ & -0.32 & 0.38 \\
\hline 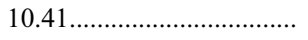 & -10.28 & $-3.67 \pm 0.02$ & $-3.79 \pm 0.02$ & $-4.30 \pm 0.04$ & -0.13 & 0.23 \\
\hline $10.41 \ldots \ldots \ldots$ & -10.09 & $-3.63 \pm 0.02$ & $-3.72 \pm 0.02$ & $-4.36 \pm 0.04$ & 0.05 & 0.18 \\
\hline $10.41 \ldots \ldots \ldots$ & -9.91 & $-3.70 \pm 0.02$ & $-3.78 \pm 0.02$ & $-4.51 \pm 0.05$ & 0.24 & 0.16 \\
\hline $10.41 \ldots \ldots \ldots \ldots \ldots \ldots \ldots$ & -9.72 & $-4.01 \pm 0.02$ & $-4.07 \pm 0.03$ & $-4.97 \pm 0.06$ & 0.43 & 0.11 \\
\hline $10.41 \ldots \ldots \ldots \ldots \ldots \ldots \ldots \ldots$ & -9.53 & $-4.38 \pm 0.03$ & $-4.45 \pm 0.03$ & $-5.24 \pm 0.08$ & 0.62 & 0.14 \\
\hline $10.41 \ldots \ldots \ldots$ & -9.34 & $-5.19 \pm 0.06$ & $-5.27 \pm 0.07$ & $-5.95 \pm 0.15$ & 0.80 & 0.17 \\
\hline $10.41 \ldots \ldots \ldots \ldots \ldots \ldots \ldots \ldots \ldots .$. & -9.16 & $-6.09 \pm 0.14$ & $-6.16 \pm 0.15$ & $-6.91 \pm 0.30$ & 0.99 & 0.15 \\
\hline $10.41 \ldots \ldots \ldots \ldots$ & -8.97 & $-6.95 \pm 0.30$ & $-6.95 \pm 0.30$ & $\ldots$ & 1.18 & $\ldots$ \\
\hline $10.41 \ldots \ldots \ldots$ & -8.78 & $-6.69 \pm 0.23$ & $-6.90 \pm 0.30$ & $-7.11 \pm 0.30$ & 1.37 & 0.38 \\
\hline $10.72 \ldots \ldots \ldots \ldots \ldots \ldots \ldots \ldots \ldots \ldots \ldots$ & -12.53 & $-5.85 \pm 0.21$ & $\ldots$ & $-5.85 \pm 0.19$ & -2.27 & 1.00 \\
\hline 10.72 & -12.34 & $-4.93 \pm 0.08$ & $\ldots$ & $-4.93 \pm 0.08$ & -2.08 & 1.00 \\
\hline $10.72 \ldots \ldots \ldots$ & -12.16 & $-4.27 \pm 0.04$ & $-6.18 \pm 0.22$ & $-4.27 \pm 0.03$ & -1.90 & 0.98 \\
\hline $10.72 \ldots \ldots \ldots$ & -11.97 & $-4.04 \pm 0.03$ & $-5.53 \pm 0.13$ & $-4.06 \pm 0.03$ & -1.71 & 0.96 \\
\hline $10.72 \ldots \ldots \ldots \ldots$ & -11.78 & $-4.07 \pm 0.02$ & $-5.56 \pm 0.14$ & $-4.09 \pm 0.02$ & -1.52 & 0.96 \\
\hline $10.72 \ldots \ldots \ldots$ & -11.59 & $-4.14 \pm 0.03$ & $-5.18 \pm 0.10$ & $-4.18 \pm 0.03$ & -1.33 & 0.90 \\
\hline $10.72 \ldots \ldots \ldots \ldots \ldots \ldots$ & -11.41 & $-4.21 \pm 0.03$ & $-5.14 \pm 0.11$ & $-4.27 \pm 0.03$ & -1.15 & 0.88 \\
\hline $10.72 \ldots \ldots \ldots \ldots \ldots \ldots \ldots$ & -11.22 & $-4.23 \pm 0.03$ & $-5.00 \pm 0.07$ & $-4.31 \pm 0.03$ & -0.96 & 0.82 \\
\hline $10.72 \ldots$ & -11.03 & $-4.18 \pm 0.03$ & $-4.80 \pm 0.06$ & $-4.30 \pm 0.03$ & -0.77 & 0.76 \\
\hline
\end{tabular}


TABLE 3-Continued

\begin{tabular}{|c|c|c|c|c|c|c|}
\hline $\log M_{\star}$ & $\log \mathrm{SFR} / M_{\star}$ & Total & $\begin{array}{c}\log \phi\left(\mathrm{SFR} / M_{\star}, M_{\star}\right)^{\mathrm{a}} \\
n_{i}<2.4\end{array}$ & $n_{i}>2.4$ & $\log \Delta \mathrm{SFR}$ & $\begin{array}{l}\text { Fraction } \\
n_{i}>2.4\end{array}$ \\
\hline 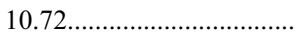 & -10.84 & $-4.15 \pm 0.03$ & $-4.66 \pm 0.05$ & $-4.32 \pm 0.03$ & -0.58 & 0.68 \\
\hline $10.72 \ldots \ldots \ldots \ldots \ldots \ldots \ldots \ldots \ldots \ldots \ldots \ldots \ldots \ldots \ldots \ldots \ldots$ & -10.66 & $-4.04 \pm 0.02$ & $-4.39 \pm 0.04$ & $-4.32 \pm 0.03$ & -0.40 & 0.53 \\
\hline 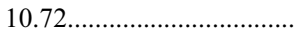 & -10.47 & $-3.89 \pm 0.02$ & $-4.17 \pm 0.03$ & $-4.21 \pm 0.02$ & -0.21 & 0.47 \\
\hline 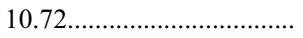 & -10.28 & $-3.83 \pm 0.02$ & $-4.05 \pm 0.02$ & $-4.24 \pm 0.03$ & -0.02 & 0.39 \\
\hline 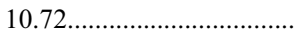 & -10.09 & $-3.82 \pm 0.02$ & $-3.99 \pm 0.02$ & $-4.33 \pm 0.03$ & 0.17 & 0.31 \\
\hline 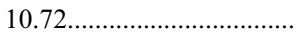 & -9.91 & $-4.09 \pm 0.02$ & $-4.20 \pm 0.02$ & $-4.75 \pm 0.04$ & 0.35 & 0.22 \\
\hline 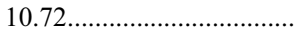 & -9.72 & $-4.52 \pm 0.03$ & $-4.62 \pm 0.03$ & $-5.21 \pm 0.06$ & 0.54 & 0.20 \\
\hline 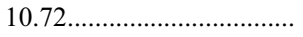 & -9.53 & $-4.95 \pm 0.04$ & $-5.02 \pm 0.04$ & $-5.81 \pm 0.11$ & 0.73 & 0.14 \\
\hline 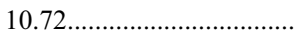 & -9.34 & $-5.70 \pm 0.09$ & $-5.81 \pm 0.10$ & $-6.50 \pm 0.18$ & 0.92 & 0.16 \\
\hline 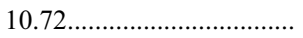 & -9.16 & $-7.12 \pm 0.30$ & $-7.12 \pm 0.30$ & $\ldots$ & 1.10 & $\ldots$ \\
\hline 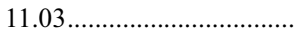 & -12.53 & $-6.04 \pm 0.22$ & $\ldots$ & $-6.04 \pm 0.21$ & -2.16 & 1.00 \\
\hline 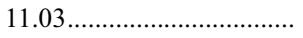 & -12.34 & $-4.82 \pm 0.06$ & $\ldots$ & $-4.84 \pm 0.06$ & -1.97 & 0.96 \\
\hline 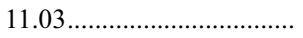 & -12.16 & $-4.34 \pm 0.03$ & $\ldots$ & $-4.35 \pm 0.03$ & -1.79 & 0.99 \\
\hline 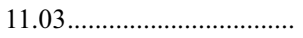 & -11.78 & $-4.28 \pm 0.02$ & $-5.94 \pm 0.15$ & $-4.29 \pm 0.02$ & -1.41 & 0.97 \\
\hline 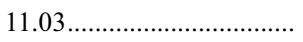 & -11.59 & $-4.35 \pm 0.02$ & $-5.87 \pm 0.11$ & $-4.37 \pm 0.02$ & -1.22 & 0.96 \\
\hline 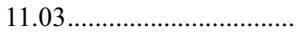 & -11.41 & $-4.46 \pm 0.02$ & $-5.63 \pm 0.10$ & $-4.49 \pm 0.03$ & -1.04 & 0.92 \\
\hline $11.03 \ldots \ldots \ldots \ldots \ldots \ldots \ldots \ldots \ldots$ & -11.22 & $-4.45 \pm 0.02$ & $-5.33 \pm 0.08$ & $-4.52 \pm 0.03$ & -0.85 & 0.86 \\
\hline 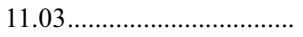 & -11.03 & $-4.46 \pm 0.02$ & $-5.33 \pm 0.08$ & $-4.53 \pm 0.02$ & -0.66 & 0.86 \\
\hline 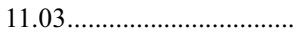 & -10.84 & $-4.38 \pm 0.02$ & $-4.99 \pm 0.06$ & $-4.50 \pm 0.03$ & -0.47 & 0.75 \\
\hline 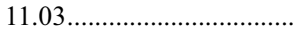 & -10.66 & $-4.32 \pm 0.02$ & $-4.85 \pm 0.04$ & $-4.47 \pm 0.02$ & -0.29 & 0.70 \\
\hline 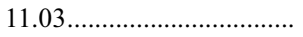 & -10.47 & $-4.24 \pm 0.02$ & $-4.62 \pm 0.03$ & $-4.47 \pm 0.03$ & -0.10 & 0.59 \\
\hline $11.03 \ldots \ldots \ldots \ldots \ldots \ldots \ldots \ldots \ldots . .$. & -10.28 & $-4.25 \pm 0.02$ & $-4.51 \pm 0.02$ & $-4.62 \pm 0.03$ & 0.09 & 0.43 \\
\hline 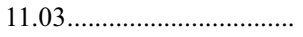 & -10.09 & $-4.40 \pm 0.02$ & $-4.64 \pm 0.03$ & $-4.79 \pm 0.03$ & 0.28 & 0.40 \\
\hline 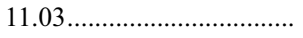 & -9.91 & $-4.79 \pm 0.03$ & $-4.96 \pm 0.04$ & $-5.30 \pm 0.05$ & 0.46 & 0.31 \\
\hline 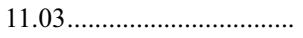 & -9.72 & $-5.36 \pm 0.05$ & $-5.50 \pm 0.07$ & $-5.95 \pm 0.09$ & 0.65 & 0.26 \\
\hline 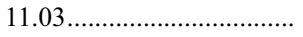 & -9.53 & $-5.90 \pm 0.08$ & $-6.00 \pm 0.10$ & $-6.58 \pm 0.16$ & 0.84 & 0.21 \\
\hline 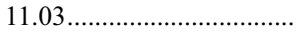 & -9.34 & $-6.31 \pm 0.15$ & $-6.42 \pm 0.16$ & $-6.99 \pm 0.30$ & 1.03 & 0.21 \\
\hline 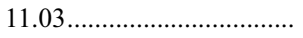 & -9.16 & $-7.39 \pm 0.30$ & $-7.39 \pm 0.30$ & $\ldots$ & 1.21 & $\ldots$ \\
\hline $11.34 \ldots \ldots \ldots \ldots \ldots \ldots \ldots \ldots \ldots . .$. & -12.34 & $-5.08 \pm 0.05$ & $\ldots$ & $-5.08 \pm 0.05$ & -1.86 & 1.00 \\
\hline 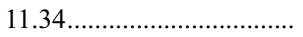 & -12.16 & $-4.74 \pm 0.03$ & $\ldots$ & $-4.75 \pm 0.03$ & -1.67 & 0.97 \\
\hline 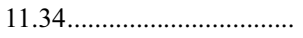 & -11.97 & $-4.68 \pm 0.03$ & $-6.48 \pm 0.19$ & $-4.69 \pm 0.03$ & -1.49 & 0.97 \\
\hline 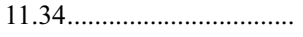 & -11.78 & $-4.82 \pm 0.03$ & $-6.57 \pm 0.21$ & $-4.83 \pm 0.03$ & -1.30 & 0.98 \\
\hline 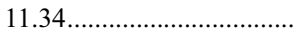 & -11.59 & $-4.98 \pm 0.03$ & $-6.31 \pm 0.15$ & $-5.00 \pm 0.03$ & -1.11 & 0.95 \\
\hline 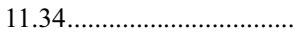 & -11.41 & $-5.00 \pm 0.03$ & $-6.12 \pm 0.10$ & $-5.03 \pm 0.04$ & -0.92 & 0.92 \\
\hline 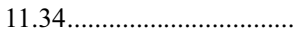 & -11.22 & $-4.95 \pm 0.03$ & $-5.89 \pm 0.10$ & $-5.01 \pm 0.04$ & -0.74 & 0.88 \\
\hline 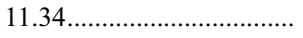 & -11.03 & $-5.15 \pm 0.04$ & $-6.07 \pm 0.11$ & $-5.21 \pm 0.04$ & -0.55 & 0.86 \\
\hline $11.34 \ldots \ldots \ldots \ldots \ldots \ldots \ldots \ldots . .$. & -10.84 & $-5.08 \pm 0.03$ & $-5.76 \pm 0.08$ & $-5.18 \pm 0.04$ & -0.36 & 0.79 \\
\hline 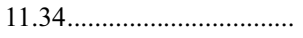 & -10.66 & $-5.00 \pm 0.04$ & $-5.56 \pm 0.08$ & $-5.15 \pm 0.04$ & -0.17 & 0.72 \\
\hline 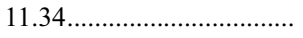 & -10.47 & $-5.05 \pm 0.04$ & $-5.52 \pm 0.05$ & $-5.24 \pm 0.04$ & 0.01 & 0.65 \\
\hline 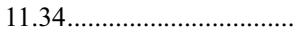 & -10.28 & $-5.16 \pm 0.04$ & $-5.53 \pm 0.06$ & $-5.43 \pm 0.05$ & 0.20 & 0.54 \\
\hline 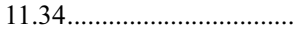 & -10.09 & $-5.56 \pm 0.06$ & $-5.77 \pm 0.07$ & $-5.98 \pm 0.08$ & 0.39 & 0.38 \\
\hline 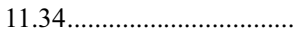 & -9.91 & $-5.90 \pm 0.08$ & $-6.14 \pm 0.10$ & $-6.29 \pm 0.14$ & 0.58 & 0.40 \\
\hline $11.34 \ldots \ldots \ldots \ldots \ldots \ldots \ldots \ldots \ldots \ldots \ldots . .$. & -9.72 & $-6.50 \pm 0.13$ & $-6.86 \pm 0.18$ & $-6.75 \pm 0.16$ & 0.76 & 0.56 \\
\hline 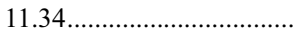 & -9.53 & $-7.30 \pm 0.18$ & $-7.30 \pm 0.18$ & $\ldots$ & 0.95 & $\ldots$ \\
\hline 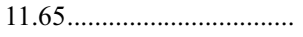 & -12.72 & $-7.25 \pm 0.30$ & $\ldots$ & $-7.25 \pm 0.30$ & -2.13 & 1.00 \\
\hline 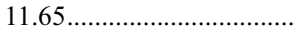 & -12.53 & $-6.58 \pm 0.19$ & $\ldots$ & $-6.58 \pm 0.18$ & -1.94 & 1.00 \\
\hline 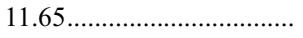 & -12.34 & $-5.73 \pm 0.07$ & $\ldots$ & $-5.73 \pm 0.07$ & -1.75 & 1.00 \\
\hline 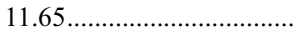 & -12.16 & $-5.54 \pm 0.05$ & $\ldots$ & $-5.54 \pm 0.05$ & -1.56 & 1.00 \\
\hline 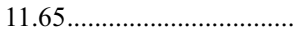 & -11.97 & $-5.70 \pm 0.05$ & $-7.29 \pm 0.18$ & $-5.71 \pm 0.05$ & -1.38 & 0.96 \\
\hline 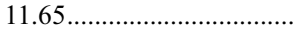 & -11.78 & $-5.87 \pm 0.07$ & $-7.13 \pm 0.20$ & $-5.89 \pm 0.07$ & -1.19 & 0.95 \\
\hline 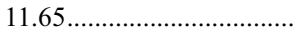 & -11.59 & $-6.16 \pm 0.10$ & $\ldots$ & $-6.16 \pm 0.10$ & -1.00 & 1.00 \\
\hline 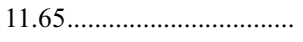 & -11.41 & $-6.23 \pm 0.10$ & $-7.52 \pm 0.30$ & $-6.25 \pm 0.10$ & -0.81 & 0.95 \\
\hline 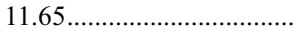 & -11.22 & $-6.03 \pm 0.09$ & $-6.43 \pm 0.17$ & $-6.26 \pm 0.10$ & -0.63 & 0.60 \\
\hline 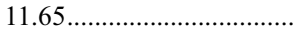 & -11.03 & $-6.20 \pm 0.09$ & $-7.59 \pm 0.30$ & $-6.23 \pm 0.09$ & -0.44 & 0.92 \\
\hline 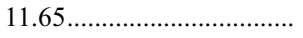 & -10.84 & $-6.48 \pm 0.13$ & $-7.15 \pm 0.30$ & $-6.58 \pm 0.12$ & -0.25 & 0.79 \\
\hline $11.65 \ldots \ldots \ldots \ldots \ldots \ldots \ldots \ldots . .$. & -10.66 & $-6.39 \pm 0.11$ & $-6.82 \pm 0.18$ & $-6.63 \pm 0.14$ & -0.06 & 0.57 \\
\hline 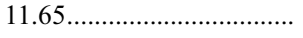 & -10.47 & $-6.61 \pm 0.13$ & $-7.04 \pm 0.21$ & $-6.81 \pm 0.16$ & 0.12 & 0.63 \\
\hline 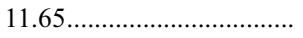 & -10.28 & $-7.16 \pm 0.23$ & $\ldots$ & $-7.16 \pm 0.21$ & 0.31 & 1.00 \\
\hline
\end{tabular}

${ }^{\text {a }}$ Units of $\phi\left(\mathrm{SFR} / M_{\star}, M_{\star}\right)$ in $\mathrm{Mpc}^{-3} \mathrm{bin}^{-1}$, where each bin is 0.3 dex wide in $M_{\star}$ and 0.1875 dex wide in SFR $/ M_{\star}$. 

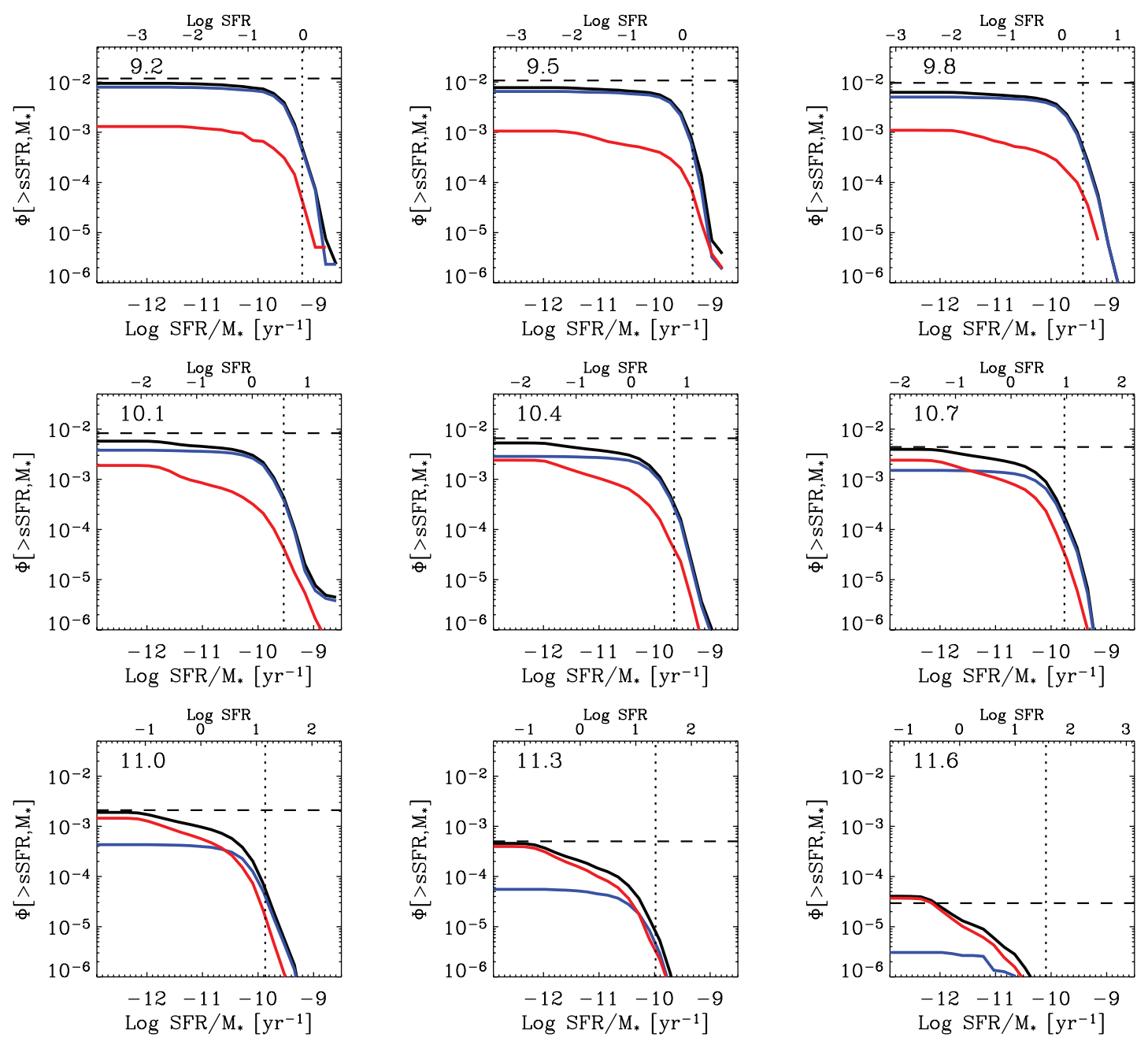

FIG. 24. - Integrated galaxy density distribution ( $1 / V_{\max }$-weighted) for all galaxies that exceed SFR $/ M_{\star}$ in a given bin of fixed $\log M_{\star}$ for subsamples plotted in Fig. 19 (see caption). Upper axis denotes $\log$ SFR using average $\left\langle\log M_{\star}\right\rangle$ (upper left corner of each box), with the vertical dotted line showing the log $\Delta_{\text {SFR }}=0.5$ for corresponding $\left\langle\log M_{\star}\right\rangle$. Black horizontal dashed line: Total galaxy density in stellar mass bin taken from local stellar mass function of Bell et al. (2003b) and Borch et al. (2006).

galaxy counts). Comparison merger rate densities are taken from the Millenium Survey (De Propris et al. 2005) and SDSS LRGs (Masjedi et al. 2006), and merger fractions are taken from De Propris et al. (2005) and Bell et al. (2006). For De Propris et al. (2005) and Masjedi et al. (2006), we estimated a stellar mass limit based on their luminosity cut. We also provide comparison points from a recent theoretical prediction (Maller et al. 2006). We find reasonably good agreement with the numbers that result from our scenario.

The total flux of stellar mass transitioning off of the SF sequence can be defined as

$$
\dot{\rho}_{\star, \downarrow}\left(M_{\star} d M_{\star}\right)=\frac{\eta_{\downarrow}}{\eta_{\text {burst }}} \frac{M_{\star} \Phi\left(>\Delta_{\mathrm{SFR}}, M_{\star}\right)}{\tau_{\text {burst }}} d M_{\star}
$$

where $\eta_{\downarrow}$ denotes the fraction of galaxies that will not return to the SF sequence, and $\eta_{\text {burst }}$ is a completeness factor that takes into account the flux from galaxies missed by this method (we adopt $\eta_{\downarrow} \sim 1$ and $\eta_{\text {burst }} \sim 1$ ).

In Figure 26 we plot (for each $M_{\star}$ bin) the total derived stellar mass flux that results from bulge-dominated galaxies with enhanced star formation. Evidence suggests that the stellar mass function of the SF sequence has remained relatively constant with time (Faber et al. 2007; Bell et al. 2007 etc.) Therefore, we would expect that star-forming galaxies should not be moving from the SF sequence any faster than the rate at which new stars are being created. Given the considerable assumptions, comparison of the two suggest good agreement, although the star-forming bulge-dominated stellar mass flux distribution is shifted to slightly higher $M_{\star}$. In addition, we compare these results with the stellar mass flux from the blue to red sequence (across the "green valley") determined in Paper III. We find a considerably lower stellar mass flux, although the distributions agree quite well. The offset between the stellar mass flux measurements coming from each side of the SF sequence could result from factors such as the time delay; "green valley" galaxies may have had their bursts several Gyr in the past, when they may have been more numerous. The differences between the two measurements may also provide some insight into the number of "hidden" (nonbursting or heavily obscured) quenched galaxies, or alternatively, the timescales over which transformations from disk to bulge-dominated might take place.

An important difference between the present analysis and the one in Paper III is that the latter makes use of both the volume density and the timescales implied by a galaxy's position in the color-magnitude diagram and spectral indices $D_{n}(4000)$ and $\mathrm{H} \delta_{A}$, whereas this work only assumes the relevant timescale. Our value 

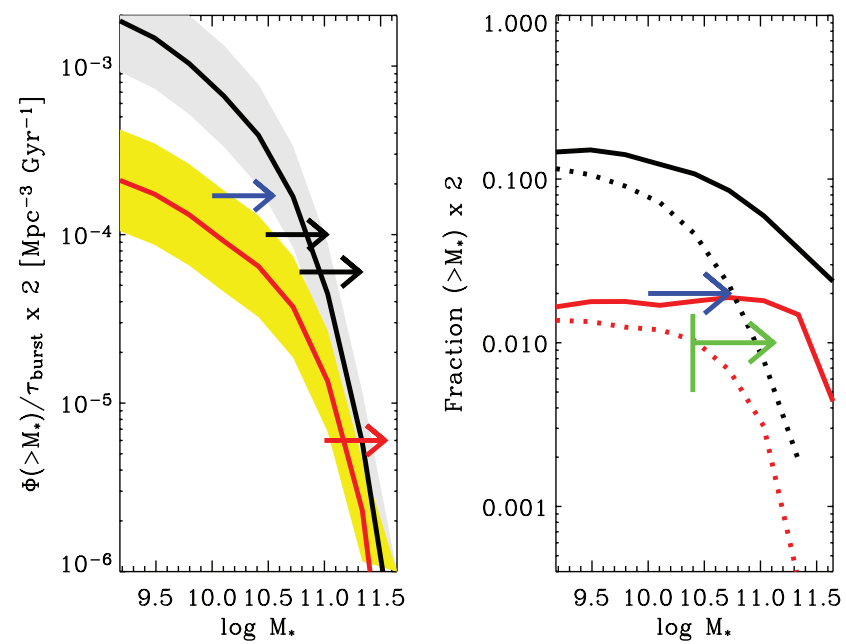

FIG. 25.—Left: Estimated "burst" rate density $(\times 2)$ for galaxies above a given $M_{\star}$, compared to various merger rates from the literature. Solid red line: Rate density using bulge-dominated galaxies with $\log \Delta_{\mathrm{SFR}}>0.5$. Solid black line: Total rate density for all galaxies with $\log \Delta_{\mathrm{SFR}}>0.5$. Arrows: Local measurements from De Propris et al. (2005; blue), Masjedi et al. (2006; red), and model prediction from Maller et al. (2006; black). Right: Fraction of galaxies $(\times 2)$ experiencing $\log \Delta_{\mathrm{SFR}}>0.5$ compared with local merger fraction from literature. Colors as above. Fraction is calculated using integrated number density of galaxies with $>M_{\star}$ (solid line) and $>M_{\star} / 2$ (dashed line) to account for possible range in merger mass ratios. Arrows: Local measurements from De Propris et al. (2005; blue) and Bell et al. (2006; green).

of $0.5 \mathrm{Gyr}$ is based on burst timescales calculated for mergers (e.g., Hopkins et al. 2006). Some studies suggest that the period of elevated SFR might be shorter ( 200 Myr) or longer, which would raise or lower our estimate. It would not be hard to incorporate additional morphological (e.g., asymmetry, M20), dynamical (velocity dispersions, mass-dependent quantities), or recent star formation history information in order to generate an improved estimate of the relevant $M_{\star}$-dependent timescale (as well as $\eta_{\downarrow}, \eta_{\text {burst }}$ ); however, this is beyond the scope of the present study.

Both this work and Paper III demonstrate a number of new applications for the UV-optical color magnitude diagram and associated physical properties and distributions. Ultimately, it is a combination of approaches that (1) compare the evolution of the SFR $/ M_{\star}$ vs. $M_{\star}$ distribution at different redshifts, and (2) use physically motivated timescales to predict the rate of change within a given time slice, which will lead to significant progress in modeling and measuring a complete history of star formation and morphological transformation.

\section{CONCLUSIONS}

We have generated a catalog of galaxies in the local $(z<0.25)$ universe with a combination of UV-optical photometry, spectroscopic measures, structural parameters, and value-added and physical quantities, and have used it for an investigation into the distribution of star formation across galaxies of different morphologies and stellar masses. Our chief results are as follows.

1. We have derived a new set of physical properties of the galaxies in our samples, including star formation and stellar mass rates and surface densities, dust attenuations, and gas fractions. Our measurements incorporate a slightly modified prescription for dust attenuation, designed to use the best available data to derive star formation rates across the whole galaxy sample. In general, this follows most closely the approaches described in Johnson

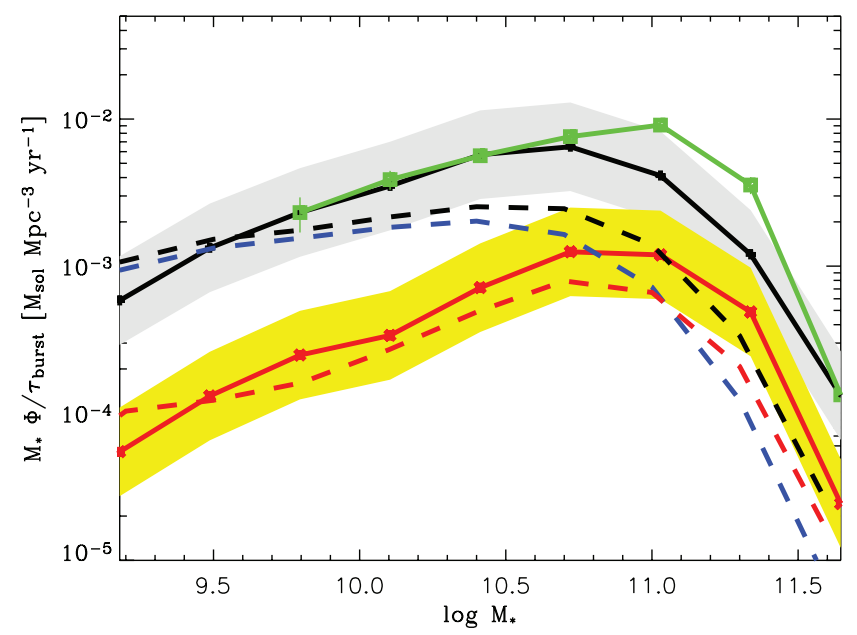

FIG. 26.-Estimated stellar mass flux density off of the SF sequence and comparison with the SFR density vs. $M_{\star}$. Solid lines: Total stellar mass flux density for all galaxies (black) and bulge-dominated galaxies (red) with $\log \Delta \mathrm{SFR}>0.5$. Green dotted line: Stellar mass flux rate for transition galaxies from Paper III (Table 3). Dashed lines: SFR density vs. $M_{\star}$ for total (black), disk-dominated (blue), and bulge-dominated (red) subsamples. Values are per $0.3 \operatorname{dex} M_{\star}$ bin.

et al. (2007) and Kauffmann et al. (2003a), but ultimately we hope to develop it as a refinement over current methodology.

2. For the first time, we have measured the local UV luminosity function against galaxy structural parameters as well as inclination. Among our key results is that we have shown that the fraction of intermediate and early-type galaxies is highest for the most UV luminous galaxies, dropping off to low fractions for the least luminous galaxies.

3. Throughout this study, our emphasis has been on the properties of galaxies on and off of a local "star-forming sequence" defined by $\log \mathrm{SFR} / M_{\star}=-0.36 \log M_{\star}-6.4$. We find, among other trends, that our measure of the star formation rate surface density, $\Sigma_{\mathrm{SFR}}$ (measured within $r_{u, 50}$ ) is nearly constant along this sequence.

4. We have split our sample into disk and bulge-dominated galaxies using the $i$-band Sersic index, and find that disk galaxies occupy a very tight locus in SFR vs. $M_{\star}$ space, while bulgedominated galaxies display a much larger spread of SFRs at fixed stellar mass. In particular, a significant fraction of galaxies with SFR and $\Sigma_{\text {SFR }}$ above those on the "star-forming sequence" are bulge-dominated.

5. We have used our derived distribution functions to ask whether a significant fraction of these galaxies may be experiencing a final episode of star formation (possibly induced by merger of other bursts), soon to be quenched, by determining whether this population can explain the growth rate of the non-star-forming population We find that this is a plausible scenario for bulgedominated galaxies near the characteristic transition mass under reasonable assumptions regarding quenching timescales. We use this technique to estimate the rate of mergers/starbursts that take galaxies off of the star-forming sequence and show that the implied merger rates are consistent with local measurements.

D. S. gratefully acknowledges discussions with Eric Bell and Michael Blanton and the hospitality of the Max Planck Institut für Astronomie in Heidelberg and the Aspen Center for Physics. This work has made extensive use of the idlutils, kcorrect, 
and Goddard IDL libraries, as well as the MPA/JHU and the NYU SDSS value-added catalogs. GALEX (Galaxy Evolution Explorer) is a NASA Small Explorer, launched in April 2003. We gratefully acknowledge NASA's support for the construction, operation, and science analysis for the GALEX mission, developed in cooperation with the Centre National d'Etudes Spatiales of France and the Korean Ministry of Science and Technology.

Facilities: GALEX

\section{APPENDIX}

\section{DEPENDENCE ON VIEW ANGLE: INCLINATION, DUST ATTENUATION, AND GALAXY STRUCTURE}

In the absence of dust attenuation (scattering and absorption), the far-field integrated photometric properties of galaxies should have no angular dependence $[F(\theta, \phi)=$ const $]$. However, even modest amounts of dust can have a considerable impact on the distribution of emitted flux. A simple axisymmetric disk geometry for light and dust would result in axisymmetry for the light distribution $[F(\theta, \phi)=$ $F(\phi)]$. In cases where galaxy axis ratios can be used to deduce the viewing angle, one can incorporate model assumptions to derive the emitted and intrinsic luminosity of a galaxy given a measurement along a single line of sight. More complex dust geometries will naturally require more detailed modeling of the three-dimensional distribution of stars and dust and the resulting two-dimensional radiation field (e.g., Jonsson et al. 2006). We make no attempt here to consider the broad set of possible attenuation curves and dust geometries that might impact our measurements in the ultraviolet. Instead, we choose to highlight two results from our study and discuss possible implications.

In Figure 27 we show the luminosity function split by observed axis ratio. We have split the sample at axis ratio $q_{25}=b_{25} / a_{25}=0.6$. Even though we have made no attempt to distinguish between disks and bulge-dominated galaxies, it is clear that the observed axis ratio has a considerable effect on the luminosity function. While the shape of the LF is largely preserved, the low axis ratio (highly inclined) subset are $0.5 \mathrm{mag}$ less luminous than the high axis ratio (low-inclination) subsample. It is tempting to assume that a proper dust-attenuation correction should remove this discrepancy due to an apparent view angle effect. However, this assumption is not valid in practice, because the distribution of axis ratios (observed or intrinsic) is known to correlate with galactic structure (Binney \& de Vaucouleurs 1981), and is not likely to be independent of intrinsic luminosity and/or other physical properties.
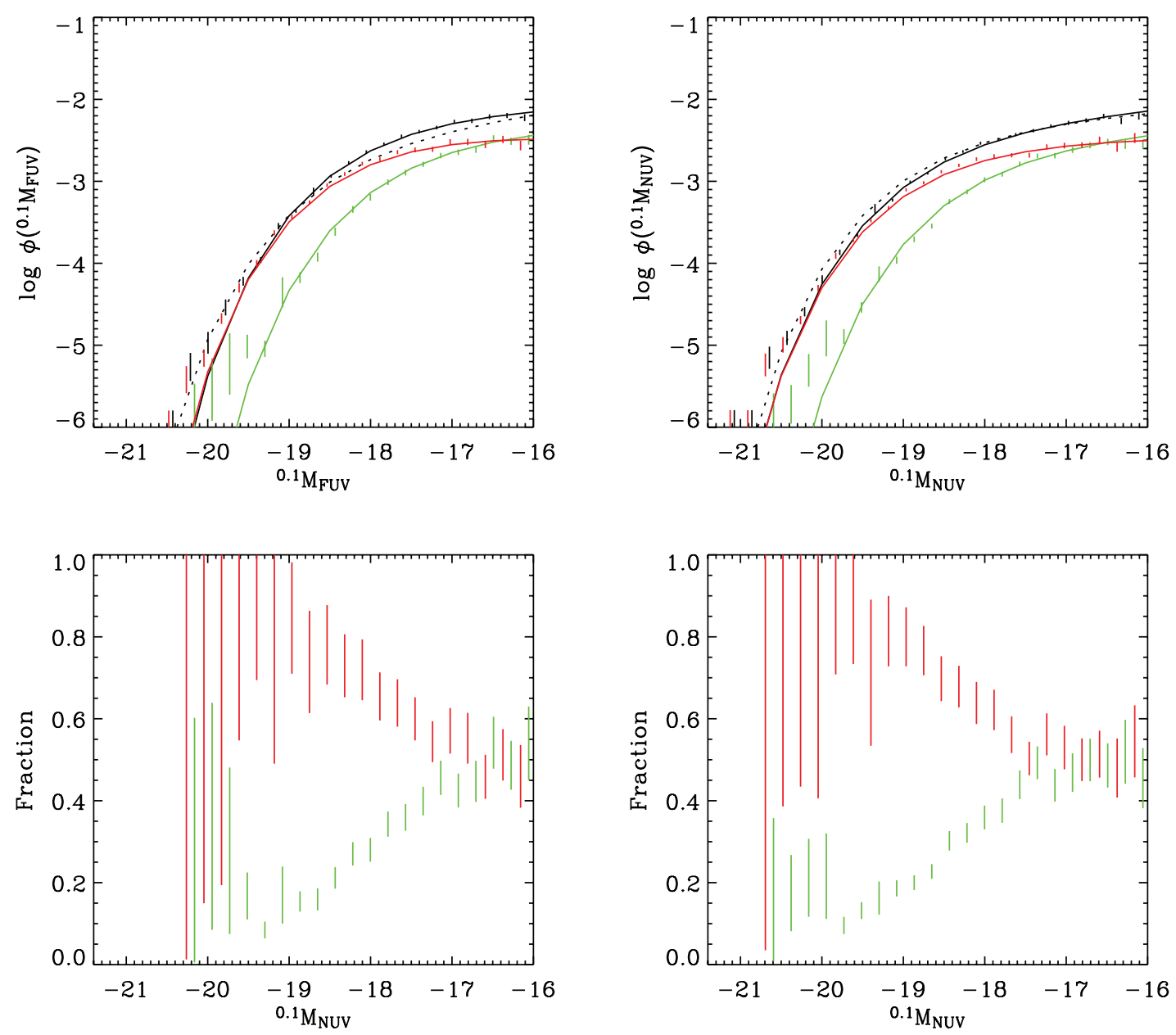

FIG. 27.-Top: FUV and NUV luminosity function for complete sample (black) and subsamples split by axis ratio: $q_{25}=b_{25} / a_{25}<0.6($ green $)$ and $b_{25} / a_{25}>0.6$ (red). Units of $\phi$ are in $\mathrm{Mpc}^{-3} \mathrm{mag}^{-1}$. The dotted curve is from the Wyder et al. (2005) and Treyer et al. (2005) LF. Bottom: Relative fraction (1/ $V_{\max }$-weighted) of low and high axis ratio vs. total. 

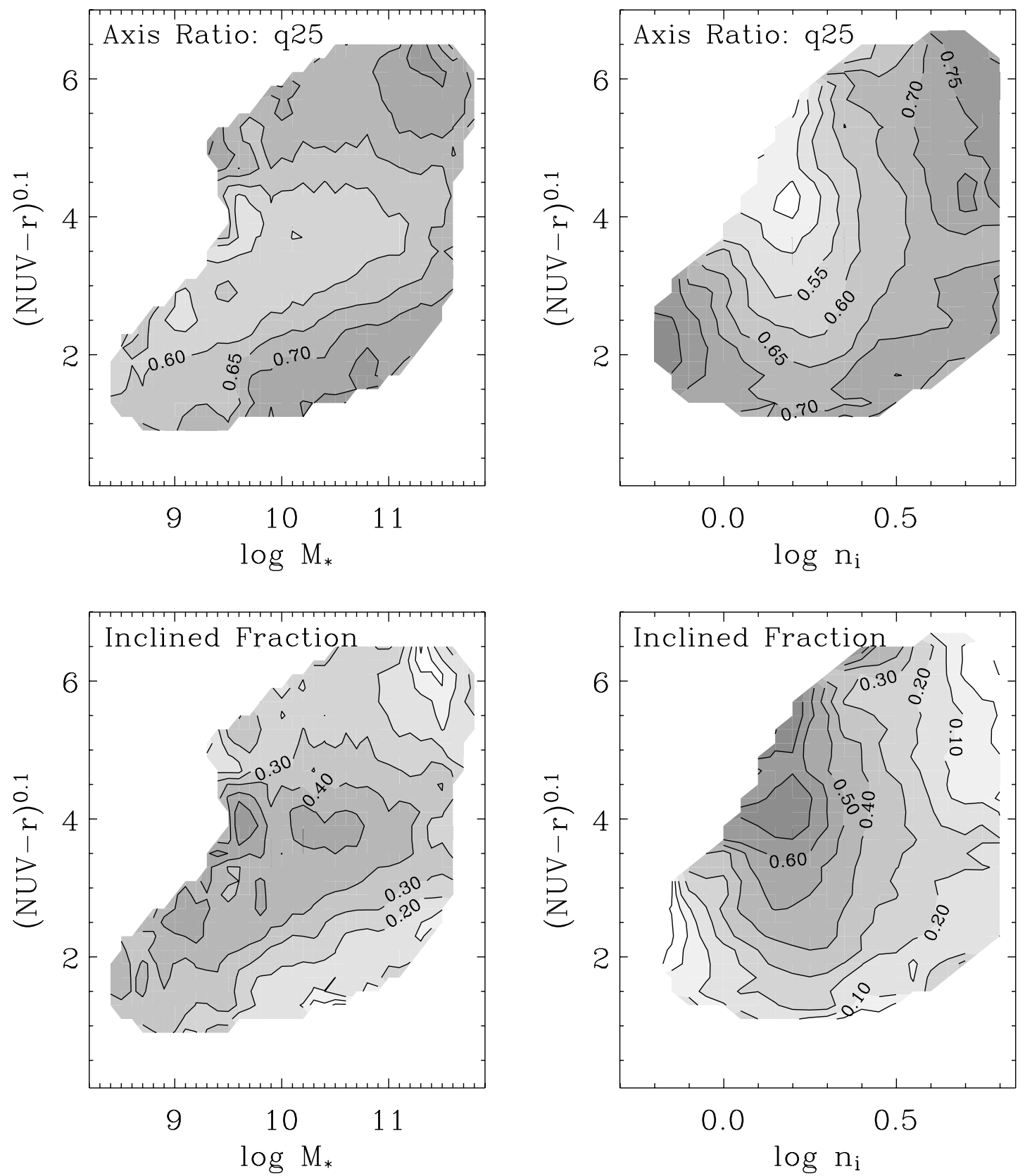

FIG. 28. - Weighted mean of axis ratio $q_{25}$ (top) and inclined fraction (bottom) in color vs. $M_{\star}\left(\right.$ left) and color vs. $\log n_{i}$ diagrams $(r i g h t)$. 
Two aspects of this are demonstrated in Figure 28, where we show the mean axis ratio, $q_{25}$, and fraction of inclined galaxies with $q_{25}<0.6$ in NUV $-r$ vs. stellar mass and NUV $-r$ vs. Sersic index planes. The plots on the left hand side shows that the distribution of inclinations is highly peaked in the observational "green valley." A likely explanation is that most of these galaxies are SF-sequence galaxies that have a higher dust attenuation at our viewing angle and therefore are more highly reddened than their less inclined counterparts. This would appear to be supported by Figure 11, which shows that this effect becomes less dramatic after application of a dust-attenuation correction.

The plots on the right of Figure 28 show that the highest axis ratios are also found in those galaxies with intermediate Sersic indices, and that the distribution of axis ratios is clearly dependent on galaxy structure. This may be due to the fact that at high (or low) Sersic index, galaxies tend to be bulge-dominated (or irregulars), with intrinsic axis ratios that differ from disks and disk/bulge composites with intermediate Sersic indexes. A dust-attenuation correction may put most of the intermediate Sersic index galaxies on the SF sequence, but it will not change the overall trend in axis ratio distribution in this plot. This, combined with the fact that the dust-attenuation properties of galaxies are likely to vary with structure (Pierini et al. 2004), suggests that conclusions drawn from the distribution of axis ratios alone should be treated with caution. Since measures of inclination itself are strongly dependent on galaxy type, they should only be used in conjunction with a suitable structural quantity.

\section{REFERENCES}

Adelman-McCarthy, J. K., et al. 2006, ApJS, 162, 38

Agüeros, M. A., et al. 2005, AJ, 130, 1022

Bell, E. F. 2002, ApJ, 577, 150 2003a, ApJ, 586, 794

Bell, E. F., \& de Jong, R. S. 2000, MNRAS, 312, 497

Bell, E. F., McIntosh, D. H., Katz, N., \& Weinberg, M. D. 2003b, ApJS, 149, 289

Bell, E. F., Phleps, S., Somerville, R. S., Wolf, C., Borch, A., \& Meisenheimer, K. 2006, ApJ, 652, 270

Bell, E. F., Zheng, X. Z., Papovich, C., Borch, A., Wolf, C., \& Meisenheimer, K. 2007, ApJ, 663, 834

Bell, E. F., et al. 2005, ApJ, 625, 23

Bianchi, L., et al. 2007, ApJS, 173, 659

Binney, J., \& de Vaucouleurs, G. 1981, MNRAS, 194, 679

Blanton, M. R. 2006, ApJ, 648, 268

Blanton, M. R., \& Roweis, S. 2007, AJ, 133, 734

Blanton, M. R., et al. 2003a, AJ, 125, 2348

2003b, ApJ, 592, 819

- 2003c, ApJ, 594, 186

. 2005a, ApJ, 629, 143

. 2005b, AJ, 129, 2562

Borch, A., et al. 2006, A\&A, 453, 869

Boselli, A., Gavazzi, G., Donas, J., \& Scodeggio, M. 2001, AJ, 121, 753

Brinchmann, J., Charlot, S., White, S. D. M., Tremonti, C., Kauffmann, G., Heckman, T., \& Brinkmann, J. 2004, MNRAS, 351, 1151

Bruzual, G., \& Charlot, S. 2003, MNRAS, 344, 1000

Calzetti, D., Armus, L., Bohlin, R. C., Kinney, A. L., Koornneef, J., \& StorchiBergmann, T. 2000, ApJ, 533, 682

Cattaneo, A., et al. 2007, MNRAS, 377, 63

Dahlen, T., Mobasher, B., Dickinson, M., Ferguson, H. C., Giavalisco, M.,

Kretchmer, C., \& Ravindranath, S. 2007, ApJ, 654, 172

Dale, D. A., et al. 2007, ApJ, 655, 863

De Lucia, G., Springel, V., White, S. D. M., Croton, D., \& Kauffmann, G. 2006, MNRAS, 366, 499

De Propris, R., Liske, J., Driver, S. P., Allen, P. D., \& Cross, N. J. G. 2005, AJ, 130,1516

de Zeeuw, T., \& Franx, M. 1991, ARA\&A, 29, 239

Driver, S. P., et al. 2006, MNRAS, 368, 414

Erb, D. K., Steidel, C. C., Shapley, A. E., Pettini, M., Reddy, N. A., \& Adelberger, K. L. 2006, ApJ, 646, 107

Faber, S. M., et al. 2007, ApJ, 665, 265

Fabian, A. C., Nulsen, P. E. J., \& Canizares, C. R. 1982, MNRAS, 201, 933

Feulner, G., Hopp, U., \& Botzler, C. S. 2006, A\&A, 451, L13

Haynes, M. P., \& Giovanelli, R. 1984, AJ, 89, 758

Heckman, T. M., et al. 2005, ApJ, 619, L35

Hoopes, C. G., et al. 2007, ApJS, 173, 441

Hopkins, A. M., \& Beacom, J. F. 2006, ApJ, 651, 142

Hopkins, P. F., Hernquist, L., Cox, T. J., Di Matteo, T., Robertson, B., \& Springel, V. 2006, ApJS, 163, 1

Jansen, R. A., \& Kannappan, S. J. 2001, Ap\&SS, 276, 1151

Johnson, B. D., et al. 2006, ApJ, 644, L109

. 2007, ApJS, 173, 377

Jonsson, P., Cox, T. J., Primack, J. R., \& Somerville, R. S. 2006, ApJ, 637, 255
Kannappan, S. J. 2004, ApJ, 611, L89

Kauffmann, G., White, S. D. M., \& Guiderdoni, B. 1993, MNRAS, 264, 201 Kauffmann, G., et al. 2003b, MNRAS, 341, 33

. 2003c, MNRAS, 341, 54

2003c, MNRAS, 346, 1055 2007, ApJS, 173, 357

Kennicutt, R. C., Jr. 1998a, ApJ, 498, 541 1998b, ARA\&A, 36, 189

Kereš, D., Katz, N., Weinberg, D. H., \& Davé, R. 2005, MNRAS, 363, 2

Knapp, G. R., Gunn, J. E., \& Wynn-Williams, C. G. 1992, ApJ, 399, 76

Kong, X., Charlot, S., Brinchmann, J., \& Fall, S. M. 2004, MNRAS, 349, 769

Labbé, I., et al. 2007, ApJ, 665, 944

Larson, R. B., \& Tinsley, B. M. 1978, ApJ, 219, 46

Maller, A. H., Katz, N., Kereš, D., Davé, R., \& Weinberg, D. H. 2006, ApJ, 647, 763

Martin, C. L., \& Kennicutt, R. C., Jr. 2001, ApJ, 555, 301

Martin, D. C., et al. 2005, ApJ, 619, L1 2007, ApJS, 173, 342 (Paper III)

Masjedi, M., et al. 2006, ApJ, 644, 54

Mathews, W. G., \& Brighenti, F. 2003, ARA\&A, 41, 191

Melbourne, J., Koo, D. C., \& Le Floc'h, E. 2005, ApJ, 632, L65

Menanteau, F., et al. 2005, ApJ, 620, 697

Meurer, G. R., Heckman, T. M., \& Calzetti, D. 1999, ApJ, 521, 64

Morrissey, P., et al. 2005, ApJ, 619, L7 2007, ApJS, 173, 682

Noeske, K. G., et al. 2007a, ApJ, 660, L43 2007b, ApJ, 660, L47

Pierini, D., Gordon, K. D., Witt, A. N., \& Madsen, G. J. 2004, ApJ, 617, 1022

Reddy, N. A., Steidel, C. C., Fadda, D., Yan, L., Pettini, M., Shapley, A. E., Erb, D. K., \& Adelberger, K. L. 2006, ApJ, 644, 792

Rich, R. M., et al. 2005, ApJ, 619, L107

Roberts, M. S., \& Haynes, M. P. 1994, ARA\&A, 32, 115

Salim, S., et al. 2005, ApJ, 619, L39

Salim, S., et al. 2007, ApJS, 173, 267 (S07)

Schmidt, M. 1959, ApJ, 129, 243

Searle, L., Sargent, W. L. W., \& Bagnuolo, W. G. 1973, ApJ, 179, 427

Seibert, M., et al. 2005, ApJ, 619, L55

Shen, S., Mo, H. J., White, S. D. M., Blanton, M. R., Kauffmann, G., Voges, W., Brinkmann, J., \& Csabai, I. 2003, MNRAS, 343, 978

Solanes, J. M., Manrique, A., García-Gómez, C., González-Casado, G., Giovanelli,

R., \& Haynes, M. P. 2001, ApJ, 548, 97

Somerville, R. S., et al. 2007, ApJ, in press (astro-ph/0612428)

Stringer, M. J., \& Benson, A. J. 2007, MNRAS, submitted (astro-ph/0703380)

Tinsley, B. M. 1968, ApJ, 151, 547

Tremonti, C. A., Moustakas, J., \& Diamond-Stanic, A. M. 2007, ApJ, 663, L77

Tremonti, C. A., et al. 2004, ApJ, 613, 898

Treyer, M., et al. 2005, ApJ, 619, L19

Vincent, R. A., \& Ryden, B. S. 2005, ApJ, 623, 137

Wyder, T. K., et al. 2005, ApJ, 619, L15 2007, ApJS, 173, 293 (Paper I)

Yi, S. K., et al. 2005, ApJ, 619, L111

Zheng, X. Z., Dole, H., Bell, E. F., Le Floc'h, E., Rieke, G. H., Rix, H.-W., \& Schiminovich, D. 2007, ApJ, 670, in press (arXiv:0706.0003) 